\title{
Ion Extraction and Counting System for Barium Tagging in Gaseous Xenon for the Enriched Xenon Observatory
}

\author{
by \\ Maria Paula Rozo Martinez
}

A thesis submitted to the Faculty of Graduate and Postdoctoral Affairs

in partial fulfillment of the requirements for the degree of

Master of Science

in

Physics

Ottawa-Carleton Institute for Physics
Department of Physics
Carleton University
Ottawa, ON, Canada

(C) 2013

Maria Paula Rozo Martinez 



\section{Abstract}

The Enriched Xenon Observatory is an experiment in the search of the neutrinoless double beta decay of ${ }^{136} \mathrm{Xe}$. A positive observation and measurement of the decay rate would determine fundamental properties of the neutrinos, such as their nature (Majorana or Dirac) and their absolute mass scale. A liquid xenon time projection chamber is currently operating at the Waste Isolation Pilot Plant (WIPP) in Carlsbad, NM; and a gaseous xenon TPC prototype is in a research and development stage at Carleton University.

The identification of the barium daughter product of the decay via laser induced fluorescence would allow a background free measurement of the process and would improve the detection sensitivity to reach neutrino masses on the order of $10 \mathrm{meV}$. As one of the main components of the barium tagging concept in gaseous xenon, a mass spectrometry system consisting of ion extraction, transport, mass identification and counting devices was designed, developed and characterized for this work. The implementation of a nanoelectrospray ionization source coupled to micron sized insulated nozzles for ion extraction from high pressure gas into vacuum of both singly and doubly charged states of barium was demonstrated; such technique limited gas flows into the system down to $\sim 20 \mathrm{ml} / \mathrm{min}$. Efficient ion transport was achieved using a sextupole ion guide and an Einzel lens for ion focusing into a quadrupole

mass analyzer. Achieved intensities were in the order of $\sim 1 \times 10^{5}$ counts per second for both barium charged states with mass resolutions of $\sim 0.1400$. 


\section{Acknowledgements}

I would like to thank all the people that contributed in the achievement of this work. I express a sincere gratitude to my supervisors, Dr. David Sinclair and Dr. Kevin Graham, whose guidance, counseling and advice made this thesis possible. Special thanks to Dr. Thomas Koffas for the opportune comments and for being an example of hard work and dedication. Thanks to the members of the evaluating committee for the constructive comments. Philippe Gravelle and Yves Baribeau, thank you for the patience and the time spent helping me solve the infinite technical issues I encountered every day. Thanks to my co-workers and friends, Ryan Killick, Etienne Rollin, Vincent Basque, Caio Licciardi, Matt Dunford and Francois Leonard. To all the EXO collaborators I met shifting in New Mexico, thanks for the shared knowledge and for the good times. To my family, the greatest gratitude for the unconditional support, for believing in me, and for filling my life with happiness. 


\section{Contents}

Abstract $\quad$ i

$\begin{array}{ll}\text { Acknowledgements } & \text { ii }\end{array}$

List of Figures $\quad$ vi

List of Tables $\quad$ x

1 Introduction $\quad 1$

1.0.1 Author's contribution ............... 3

2 Neutrinos 4

2.1 Historical Background . . . . . . . . . . . . . . . 5

2.1.1 The Nuclear Model . . . . . . . . . . . . . . . . 5

2.1.2 Postulation of the Neutrino . . . . . . . . . . . . 6

2.1.3 Neutrino Flavors . . . . . . . . . . . . . . . 7

2.1.4 The Solar Neutrino Problem . . . . . . . . . . . . . 8

2.2 Neutrino Oscillations . . . . . . . . . . . . . . . . . 9

2.2.1 The $V_{P M N S}$ Mixing Matrix . . . . . . . . . . . . . . . . . 10

2.2.2 Two Neutrino Mixing . . . . . . . . . . . . . . . . . . . . . . 10

2.2.2.1 Survival Probability . . . . . . . . . . . . . 10

2.2.2.2 Neutrino Oscillation Experiments . . . . . . . . . 11

2.2 .3 Mass Hierarchy . . . . . . . . . . . . . . . . . . . . 12

2.3 Massive Neutrinos in the Standard Model . . . . . . . . . . . . . . . 13

2.3.1 Dirac Mass . . . . . . . . . . . . . . . . 13

2.3.2 Majorana Mass .................. 14 
2.3.3 Absolute Neutrino Mass . . . . . . . . . . . . . . . . 16

2.4 Double Beta Decay . . . . . . . . . . . . . . . . . . . . . 17

2.4.1 Neutrinoless Double Beta Decay . . . . . . . . . . . . . . . 18

2.4.2 Decay Rates .................. . . 20

2.4.2.1 Double Beta Decay Rate . . . . . . . . . . . . . 20

2.4.2.2 Neutrinoless Double Beta Decay Rate . . . . . . . 20

2.4.3 $0 \nu \beta \beta$ Decay Experiments . . . . . . . . . . . . 23

3 The Enriched Xenon Observatory $\quad 26$

3.1 Experimental Criteria . . . . . . . . . . . . . . 27

3.2 EXO Experiment . . . . . . . . . . . . . . . . 29

3.2.1 EXO Liquid Xenon Prototype $($ EXO-200) . . . . . . . . . . . 29

3.2.2 EXO Gaseous Xenon Prototype . . . . . . . . . . . . . . 33

3.3 Barium Tagging in a Gaseous Xe TPC . . . . . . . . . . . . . . . 35

3.3.1 Single Atom Spectroscopy . . . . . . . . . . . . . . . . . 35

3.3.2 General Concept . . . . . . . . . . . . . . . 37

3.3.2.1 Current Status . . . . . . . . . . . . 38

4 Mass Spectrometry Theory 40

4.1 Electrospray Ionization . . . . . . . . . . . . . . . . . . . . . . . . 41

4.1.1 Nanoelectrospray Ionization . . . . . . . . . . . . . . . . 43

4.1.1.1 Extractor Electrode . . . . . . . . . . . . . 44

4.1 .2 Taylor Cone Theory . . . . . . . . . . . . . . 45

4.1.2.1 The Taylor Cone . . . . . . . . . . . . . . . 46

4.1.2.2 Cone Threshold Voltage . . . . . . . . . . . . . . 48

4.1.2.3 Jet Formation . . . . . . . . . . . . . . . . . . . 49

4.1.2.4 Emission Radius . . . . . . . . . . . . . . . . 51

4.2 Multipole Ion Guides ． . . . . . . . . . . . . . . . . . . . . . . . . 51

4.2.0.5 Ion Motion in a 2D Multipole Field . . . . . . . . . . 54

4.2.1 Sextupole Ion Guide (SPIG) . . . . . . . . . . . . . . . . 55

4.2.1.1 Einzel Lens . . . . . . . . . . . . . . . . . . . 56

4.2.2 Quadrupole Mass Filter . . . . . . . . . . . . . . 56

4.2.3 Channeltron Electron Multiplier . . . . . . . . . . . . . 60 
5 Ion Counting and Extraction System $\quad 62$

5.1 Experimental Setup . . . . . . . . . . . . . . 63

5.1 .1 The Ion Source . . . . . . . . . . . . . . . . 64

5.1.2 Extractor Electrode . . . . . . . . . . . . . . . 66

$5.1 .3 \mathrm{SPIG} \ldots \ldots \ldots \ldots$. . . . . . . . . . 67

5.1.4 Quadrupole Mass Analyzer . . . . . . . . . . . . . . 68

5.1.5 Summary of Operational Parameters for the System . . . . . . 69

5.2 SIMION Calculations . . . . . . . . . . . . . . . . 71

5.2.1 Simulated Ion Extraction Efficiency . . . . . . . . . . . . . . 74

5.2 .2 Ion Kinetic Energies . . . . . . . . . . . . . . . . 75

5.2.3 Einzel Lens Distance Optimization . . . . . . . . . . . . . 77

5.3 Experimental Results . . . . . . . . . . . . . . . . . 79

5.3.1 Spraying Characteristics . . . . . . . . . . . . . . 79

5.3.2 System Calibration . . . . . . . . . . . . . . . . . 81

5.3.3 Typical Barium Acetate Fragmentation . . . . . . . . . . . . . 82

5.3.4 Skimmer Electrode Effect on $\mathrm{Ba}^{+}$and $\mathrm{Ba}^{++}$Detection . . . . 86

5.3.5 Ion Source Pressure and Geometry Effects on Ion Intensity . . 88

5.3.6 Efficiency, Resolution and Stability . . . . . . . . . . . . 90

$\begin{array}{lll}6 & \text { Summary and Conclusion } & 93\end{array}$

$\begin{array}{ll}\text { References } & 96\end{array}$ 


\section{List of Figures}

2.1 Feynman diagram for double beta decay modes. a) Double Beta Decay with emission of two antineutrinos (lepton number conserved). b) Neutrinoless Double Beta Decay (lepton number not conserved). . . . 19

2.2 Effective Majorana mass as a function of the lightest neutrino mass in the normal and inverted hierarchies. Modified from [33] . . . . . . 22

2.3 Spectra of the sum of the electron kinetic energies $K_{e}$ (endpoint $Q$ ) for the $2 \nu \beta \beta$ (dotted curve normalized to 1) and $0 \nu \beta \beta$ (solid curve normalized to $10^{-6}$ ). Taken from [67]. . . . . . . . . . . . . 23

3.1 Schematics of EXO 200 TPC. Adapted from [38] . . . . . . . . . . . 30

3.2 Schematics of the EXO 200 setup. Adapted from [38] . . . . . . . . . 32

3.3 Schematic of gaseous xenon prototype detector. Adapted from [40] . 34

3.4 Atomic level scheme for $B a^{+}$ions. Wavelength values for transitions taken from [48]. Adapted from [49]. . . . . . . . . . . . . . . 36

4.1 Diagram of the barium ion extraction and counting system. . . . . . . 40

4.2 Diagram of the electrospray ionization mechanism. Adapted from [64]. 43

4.3 Diagram of the liquid Taylor Cone. $\alpha$ is the cone angle, $p_{\gamma}$ and $p_{E}$ are the surface tension and electrostatic pressures respectively, $E_{n}$ is the electric field on the cone surface, and $R$ is the radius of curvature. Adapted from [74] . . . . . . . . . . . . . . . . . . 46

4.4 Types of jet break up as described by Hartman et al. From left to right, a symmetric, a lateral and a ramified jet break up. Adapted

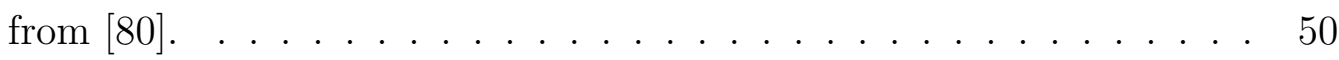


4.5 Cross section diagram of linear radio frequency multipole ion guides of order $\mathrm{n}=2,3$ and $4 . \ldots \ldots \ldots$. . . . . . . . . . . 52

4.6 Pseudopotential well model for a quadrupole, sextupole and octupole with arbitrary $r_{0}, \Omega$ and $V_{0}$ values. . . . . . . . . . . . . . . 53

4.7 Simulated stable ion trajectories for ions traveling within a sextupole and quadrupole field. . . . . . . . . . . . . . . . . . . . 54

4.8 Diagram of a sextupole. Cylindrical rods in an hexagonal array. . . . 55

4.9 Diagram of Einzel lens and corresponding ion path. The voltage polarity of the middle electrode depends on the polarity of the ion beam. 56

4.10 Diagram of a quadrupole mass filter. Ions would oscillate in the $\mathrm{x}-\mathrm{y}$ plane and travel in the $\mathrm{z}$ direction. . . . . . . . . . . . . . . . 57

4.11 Stability region for a linear quadrupole with $r_{0}=8.23 \mathrm{~mm}$ at $f=1.2$ $\mathrm{MHz}$ and $m=136$ amu. . . . . . . . . . . . . . . . . . . . . . . . . 59

4.12 Diagram of the multiplier-dynode configuration for positive ion counting. Adapted from [81]. . . . . . . . . . . . . . . . . . 60

5.1 Schematics of the mass spectrometry system. . . . . . . . . . . . . 64

5.2 Close up view of the nanoelectrospray ion source mount. . . . . . . . 65

5.3 Diagram of glass nanoelectrospray emitter. Shaded area corresponds to the conductive coating. . . . . . . . . . . . . . . . 65

5.4 Picture of the extractor electrode with mounted ceramic nozzle. . . . 66

5.5 Diagram of extractor electrode aperture geometries. . . . . . . . . . 67

5.6 Picture of SPIG setup. . . . . . . . . . . . . . . . . . . . 68

5.7 Quadrupole Mass Analyzer and CEM housing. The mount is a 6" CF. 69

5.8 Schematic of the ion extraction and counting system simulation. . . . 73

5.9 Simulated transmission efficiencies for barium ions of $136 \mathrm{amu}$ at different emission angles and operating pressures with $100 \mathrm{~V}$ potential drop across the extractor electrode. . . . . . . . . . . . . . . 74

5.10 Simulation results for ion trajectories across the skimmer plate at the entrance of the SPIG. Electric field lines in the region between the electrodes are shown. Ions are singly charged, with mass of $136 \mathrm{amu}$ and the potential drop within the skimmer is $60 \mathrm{~V}$. . . . . . . . . 75 
5.11 Calculated ion kinetic energies with respect to skimmer voltages for $\mathrm{Ba}^{+}$and $\mathrm{Ba}^{++}$ions. Uncertainties on kinetic energies are in the order of $\sim 10 \mathrm{eV}$ from the statistical variation of the energy spread. . . . . . 76

5.12 Simulation results for ion trajectories across the Einzel lens that focuses the ions into the quadrupole analyzer. Field lines in the array are drawn. Flying ions are singly charged particles of $\mathrm{m}=136 \mathrm{amu}$ with kinetic energies of $\sim 60 \mathrm{eV}$. SPIG - Einzel distance is $10 \mathrm{~mm}$. . . 77

5.13 Einzel lens parameter optimization for efficient ion transport between the SPIG exit and Quadrupole entrance. . . . . . . . . . . . . . . 78

5.14 Variation in voltage and spray current for a set of needles. Each set of connected points represents a different needle. The threshold voltage, $U_{T}$, is indicated for every case. The corresponding experimental uncertainties for current and voltage are $\pm 5 \mathrm{nA}$ and $\pm 2 \mathrm{~V}$. . . . . . 79

5.15 Spray mode identification from current voltage relation on nanoelectrospray emitters. The corresponding experimental uncertainties for current and voltage are $\pm 5 \mathrm{nA}$ and $\pm 2 \mathrm{~V}$. . . . . . . . . . . 80

5.16 Diagram of barium acetate molecule bonding. . . . . . . . . . . . . . 82

5.17 Mass spectrum of barium mono-acetate dissociation at $100 \mathrm{nA}$ of spraying current, $70 \mathrm{~V}$ and $20 \mathrm{~V}$ drop across the extractor electrode and skimmer respectively and ion source operating at 3 psig in nitrogen at $1 \mathrm{ml} / \mathrm{min}$ flow rate. Scan ranges from 60 to $120 \mathrm{~m} / \mathrm{z}$ (a), 120 to $180 \mathrm{~m} / \mathrm{z}$ (b) and 180 to $200 \mathrm{~m} / \mathrm{z}$ (c) are shown. . . . . . . . . . . .

5.18 Mass spectrum of barium (top) singly and (bottom) doubly charged obtained with the nanoelectrospray ion source. Uncertainties on intensities for this particular case are not significant to show as the variations are mainly dependent on reproducibility constraints. . . . .

5.19 Skimmer voltage effect on $\mathrm{Ba}^{+}$and $\mathrm{Ba}^{++}$normalized ion intensities. Variation in the voltage reading is of $\pm 2 \mathrm{~V}$. . . . . . . . . . . . . . . 86

5.20 Selection ability of singly or doubly charged barium ions for variations in the skimmer potential. (a) Skimmer potential $=70 \mathrm{~V}$, (b) Skimmer potential $=140 \mathrm{~V} \ldots \ldots$. . . . . . . . . . . . . . . 
5.21 $\mathrm{Ba}^{++}$counts for different potential drops across the extractor electrode for different aperture geometries. Intensity uncertainties are not significant to show as the variations are mainly dependent on reproducibility constraints. The voltage readings have corresponding uncertainties of \pm 2 V . . . . . . . . . . . . . . . . . . . . . . 88

$5.22 \mathrm{Ba}^{++}$counts for different potential drops across the extractor electrode for different ion source operating pressures. Intensity uncertainties are not significant to show as the variations are mainly dependent on reproducibility constraints. The voltage readings have corresponding uncertainties of $\pm 2 \mathrm{~V}$. . . . . . . . . . . . . . .

5.23 Average resolution of the mass analyzer for $\mathrm{Ba}^{++}$detection for different runs. . . . . . . . . . . . . . . . . . . . . . . . . . 91

5.24 Ion production stability over $2 \mathrm{~h}$ long runs at different operating pressures of the ion source. . . . . . . . . . . . . . . . . . . 92 


\section{List of Tables}

2.1 Neutrino mass splitting measurements at the $1 \sigma$ confidence level [21] 11

2.2 Neutrino mixing angle measurements at the $1 \sigma$ confidence level[21] . . 12

2.3 List of isotopes for which $2 \nu \beta \beta$ has been observed (at 68\% CL) [21]. 18

2.4 Sensitivities to $T_{1 / 2}^{0 \nu \beta \beta}$ and $\left\langle m_{\beta \beta}\right\rangle$ (for the range in nuclear matrix element calculations) reached by experiments searching for $0 \nu \beta \beta[21]$. . 24

5.1 Summary of electrode voltages and operating pressures of the system components. . . . . . . . . . . . . . . . 70

5.2 Summary of geometry parameters used for SIMION calculations compared to the experimental values. . . . . . . . . . . . . . . . 72

5.3 Spectrum peaks of CsI, RbI and Barium Acetate used for calibration of the mass spectrometer. . . . . . . . . . . . . . . . 81

5.4 Possible fragmentations of Barium Acetate . . . . . . . . . . . . . . 82

5.5 Stable barium isotopes with corresponding masses and abundances. . 84 


\section{Chapter 1}

\section{Introduction}

Over the last few decades, the observations about neutrino oscillations have proven that neutrinos are massive particles although no absolute mass measurement has been accomplished yet. Due to the weakly interacting characteristics of neutrinos, their detection is very challenging. Different experimental approaches, in particular the study of neutrinoless double beta decay, are currently in progress for the study of neutrino fundamental properties. Neutrinoless double beta decay is a rare process that if observed, not only would determine the nature of the neutrinos (Dirac or Majorana particles) but would establish their absolute mass scale from the lifetime measurement of the decay.

The Enriched Xenon Observatory is an experiment in the search for the neutrinoless double beta decay of ${ }^{136} X e$. Currently, it is operating a liquid phase time projection chamber (TPC) at the Waste Isolation Pilot Plant (WIPP) in Carlsbad, NM and has measured an upper limit for the lifetime to be $T^{0 \nu \beta \beta}>1.6 \times 10^{25} y$ at a $90 \%$ confidence level and corresponding to neutrino masses of $m_{\beta \beta}<0.14-0.38 \mathrm{eV}$. In order to probe the allowed region for the inverted neutrino mass hierarchy EXO is planning the construction of a multi-tonne detector using either gaseous or liquid Xe.

The main advantage of a gaseous xenon detector is the possibility of performing a background free measurement of the decay by identifying the barium daughter product of the reaction using laser induced fluorescence. In order to accomplish such goal it is necessary to extract the barium ion from the pressurized xenon vessel into 
vacuum for mass selection, charge conversion and transport into a quadrupole linear ion trap for ion confinement where a laser beam would excite it and the emitted photons from its de-excitation can be analyzed to perform a definitive identification of the ion species. The feasibility of a complete barium tagging concept hasn't been demonstrated yet and questions regarding its performance and limitations are still under study.

The core of this thesis is the study of the ion transport and mass identification system for the barium tagging concept in gaseous xenon. To test its operation, an ion source was designed and developed. It consists of a nanoelectrospray ionization source coupled to micron sized insulated nozzles for ion extraction from high pressure gas into vacuum. The whole setup constitutes a mass spectrometry system whose main purpose is the production, extraction, identification and counting of barium ions in both singly and doubly charged states. Questions about the feasibility of the system, its stability and its limitations in terms of operational pressures and extraction efficiencies with minimum gas flows are addressed in this work.

The thesis is divided into five principal chapters. Chapter 2 introduces the neutrinos from a historical perspective, it outlines the most important theoretical postulates and experimental observations that have established the properties that are currently known about them. Discussions about the implications of having massive neutrinos within the standard model, and about the experimental approaches to determine their nature and absolute mass scale by the study of neutrinoless double beta decay are presented. The current status of several experiments in the search of that process in a variety of isotopes is described at the end of the chapter.

Chapter 3 covers a detailed description of the liquid and gaseous xenon prototypes under operation by the EXO experiment outlining the needed criteria for the detector operation for minimum background conditions. The barium tagging concept that would allow a background free observation of the neutrinoless double beta decay in ${ }^{136} \mathrm{Xe}$ is described and a summary of its current status is presented.

Chapter 4 constitutes the theoretical background for the operation of the mass spec- 
trometry system developed for this work. It describes the principles of the nanoelectrospray ionization technique and the theory behind the ion formation mechanism. The theory on ion motion in 2D multipole fields is presented with a particular emphasis on sextupole ion guides and quadrupole mass filters.

Chapter 5 covers in detail the developed ion extraction and counting system. An outline of the experimental setup and summary of the operational parameters are included. Calculations from the SIMION simulations of the ion transport within the system and achieved experimental results and analysis are presented. Finally, a summary of the accomplished work and future plans is described in Chapter 6 .

\subsubsection{Author's contribution}

I developed a mass spectrometry system for ion selection and counting as part of a barium tagging system in gaseous Xe to be used in the EXO experiment as a means of rejecting background events in the search for the neutrinoless double beta decay of ${ }^{136} \mathrm{Xe}$. I simulated the system in the SIMION framework to evaluate the ion transmission efficiency under various electrode configurations. In conjunction with David Sinclair and Rodney Schnarr, we designed a nanoelectrospray ion source for barium ion extraction from high pressure gas into vacuum using micron sized insulated nozzles. The ion source was machined at the Science Technology Centre (STC) at Carleton University. I assembled and commissioned the setup with the technical support from Yves Baribeau (electronics) and Philippe Gravelle (mechanics). I characterized the system and tested the effects of solvent concentration, gas pressure, and electric field variation on the different electrodes for ion transfer and detection optimization and performed the corresponding analysis of the data obtained.

I also participated in the daily operations of the EXO-200 experiment by taking remote and on site shifts. They mainly involved detector and sub-systems maintenance, slow control monitoring, and calibration and low background data collection. I was a co-author of the EXO collaboration article: An improved measurement of the $2 \vee \beta \beta$ half-life of Xe-136 with EXO-200. [arXiv:1306.6106v3], 34 pages (2013) 


\section{Chapter 2}

\section{Neutrinos}

The Standard Model (SM) of particle physics describes the properties and interactions of subatomic particles. One of the particles of more interest among the scientific community is the neutrino, a weakly interacting chargeless fermion that doesn't interact via the electromagnetic or strong force making its detection difficult. Despite being one of the most abundant particles in the universe some of its fundamental properties, such as its mass and nature, are still unknown. Recent observations from neutrino oscillation experiments imply the existence of massive neutrinos, however within the SM neutrinos are considered massless particles. This disagreement has opened new questions that are tried to be explained within the physics beyond the SM framework. Experimental efforts on probing the neutrino absolute mass scale and its nature (Majorana or Dirac) are being pursued by the investigation of a particular mode of beta decay, the so called neutrinoless double beta decay. Such process violates conservation of lepton number by two units and its successful observation and measurement of its decay rate would confirm the Majorana nature of neutrinos and establish an absolute mass scale for its mass.

This chapter presents an introduction to neutrinos and their role in particle physics. Section 2.1 presents a brief historical review of the nuclear model, the motivation for the postulation of the neutrino and a description of the solar neutrino problem. The theory behind neutrino oscillations, the respective experimental observations and the effect on the neutrino mass hierarchy are presented in Section 2.2. The implications of massive neutrinos in the Standard Model and the characteristics of the Dirac and 
Majorana nature of neutrinos are elucidated in Section 2.3. Finally, the theory of neutrinoless double beta decay, the decay rate of the process and the results from current experiments on its search are depicted in Section 2.4.

\subsection{Historical Background}

\subsubsection{The Nuclear Model}

Around 1930 the nuclear model of the atom described electrons bound to a positive nucleus held together by the electromagnetic force. The nucleus was believed to be composed of protons and electrons and both atomic number (A) and nuclear charge (Z) were accounted for. One of the main problems to this depiction was the observation of spontaneous beta decay of radioactive nuclei:

$$
A \rightarrow A^{\prime}+e
$$

In this process all the emitted electrons were expected to have the same energy but instead a continuous electron energy spectrum was observed violating energy and momentum conservation.

Another problem of the model was related to spin-statistics. For example, ${ }^{14} \mathrm{~N}$ was considered to consist of a nucleus with 14 protons and 7 electrons orbited by 7 more electrons. At that time, protons and electrons were known to have spin $1 / 2$, so in such model 20 out of the 21 particles in the nucleus would pair up to cancel each other's spin, and the nucleus would have a net spin of 1/2. However, in 1929 Rosetti discovered that ${ }^{14} N$ had an integer spin; in an attempt to solve such inconsistency, Wolfgang Pauli proposed the existence of the neutron, a very light neutral particle of spin $1 / 2$ inside the nuclei. This new particle solved the spin-statistics problem and explained the continuous beta spectrum. For the first case, ${ }^{14} N$ would be a stable nuclide with both seven protons and seven neutrons, each contributing a nuclear spin of $\pm 1 / 2$ and giving the nucleus a total spin of one. For the latter case, neutrons would be emitted in conjunction with electrons and the energy spectrum of the electrons would no longer be monoenergetic. 
However, to be consistent with the observed energy spectrum, the mass of the neutron had to be less than one percent of the proton mass. This fact implied that the neutron was almost impossible to detect due to the weakness of its interaction. In 1932, the neutron was discovered at the Cavendish Laboratory of the University of Cambridge in England [1]. It was determined then that it was a strongly interacting neutral particle, and the nuclear model simplified to having neutrons and protons bound by the strong force inside the nuclei of atoms.

\subsubsection{Postulation of the Neutrino}

In 1934, Enrico Fermi reformulated the idea that a very light neutral particle (which he called the neutrino, $\nu$ ) was involved in radioactive decays; he proposed that neutrons would decay to protons via a four fermion interaction,

$$
n \rightarrow p+e^{-}+\overline{\nu_{e}}
$$

where making use of the concept of antiparticle introduced in 1931 by Dirac, the electron $e^{-}$and the electron antineutrino $\bar{\nu}_{e}$ were created as a pair. His theory predicted the existence of the inverse process to (2.2) having the same strength as the neutron decay,

$$
\overline{\nu_{e}}+p \rightarrow e^{+}+n
$$

The prediction of this process (eq (2.3)) allowed the detection of the antineutrino in 1955, where $\overline{\nu_{e}}$ scattering events were observed at the Savannah River reactor in South Carolina [2] . The experiment consisted of an intense antineutrino source from the reactor and an array of three tanks of liquid scintillator, interleaved by two tanks of water; gamma rays from positron annihilations and from neutron capture on cadmium chloride dissolved in the water were picked up by photomultiplier tubes

located around the tanks. The coincidence of this two events within a time delay of $5 \mu s$ among each other and the consistency between the measured and predicted inverse beta decay cross sections indicated the existence of antineutrinos and validated Fermi's theory on beta decay. 


\subsubsection{Neutrino Flavors}

During the subsequent years, experimental efforts led to a series of interesting findings within the field of particle physics. In 1956, at the Brookhaven National Laboratory (BNL), parity violation was observed for the first time in the decay of two species of neutral kaons [3]. This fact was later confirmed at Columbia University in an experiment on the radioactive beta decays of ${ }^{60} \mathrm{Co}$ [4]. In 1958, the neutrino was confirmed to be a left-handed particle at BNL in an experiment studying the circular polarization and resonant scattering of gamma rays following orbital electron capture in a metastable state of ${ }^{152} \mathrm{Eu}[5]$. Four years later, at the same laboratory, the existence of the muon neutrino $\nu_{\mu}$ was established after discovering that charged pions decay into muons and their associated neutrino [6]. Moreover, the discovery of the $\tau$ (tau), the heaviest electrically charged lepton [8]; and the theory describing the electromagnetic and weak interactions of particles (known as the Standard Model) gave strong evidence of the existence of a tau neutrino, $\nu_{\tau}$.

The study of the Z boson decay and measurement of its lifetime by the Large Electron Positron (LEP) collider and the Stanford Linear Accelerator Center (SLAC) in 1989 established the experimental evidence for the existence of at least three lightweight neutrino types consistent with the previous discoveries. The observations were based on the width of the mass distribution of the $\mathrm{Z}$ boson. Broader peaks would be obtained for shorter lifetimes, corresponding to a higher number of possible particles the $\mathrm{Z}$ boson could decay to. Considering the allowed decay channels of the $\mathrm{Z}$ boson, the measurements were found in agreement with the existence of three types of light neutrinos. There is no definitive proof that limits the number of neutrino flavours to three, the possibility that there could be more types remains as an open question in particle physics.

In 1964, it was shown that in the decay of neutral kaons, not only parity was violated, but the charge conjugation symmetry was violated as well [7]. This small effect, called CP symmetry breaking, opened a discussion on whether this was the cause of the matter-antimatter asymmetry at the beginning of the universe which after matter-antimatter annihilation lead matter to dominate the known universe. In 2004, the BaBar [9] and Belle [10] experiments observed CP violation in B meson 
decays, but its effect was found to be too weak to account for such asymmetry.

\subsubsection{The Solar Neutrino Problem}

In the decade of 1930, Bethe and Critchfield proposed a mechanism by which the sun generated energy. The proposed fusion reaction, also known as the pp chain explains how protons make alpha particles in the sun. There are five reactions responsible for neutrino production at different stages of the pp chain. They are

$$
\begin{gathered}
p+p \rightarrow d+e^{+}+\nu_{e} \\
p+p+e^{-} \rightarrow d+\nu_{e} \\
{ }^{3} \mathrm{He}+p \rightarrow \alpha+e^{+}+\nu_{e} \\
{ }^{7} \mathrm{Be}+e^{-} \rightarrow{ }^{7} \mathrm{Li}+\nu_{e} \\
{ }^{8} \mathrm{~B} \rightarrow{ }^{8} \mathrm{~B} e^{*}+e^{+}+\nu_{e}
\end{gathered}
$$

The neutrinos coming out from such reactions all have a characteristic energy spectrum. The majority of neutrinos are produced from the initial pp reaction (Flux $\sim 10^{11} \mathrm{~cm}^{-2} \mathrm{~s}^{-1}$ ), however because they have energies $<1 \mathrm{MeV}$ most detectors are insensitive for their observation. For such reason, most experiments work with the neutrinos produced in the ${ }^{8} B$ reaction, which despite their less abundance $\left(\right.$ Flux $\left.\sim 10^{3} \mathrm{~cm}^{-2} \mathrm{~s}^{-1}\right)$ they reach energies up to $15 \mathrm{MeV}$.

In 1964, Raymond Davis at BNL proposed an experiment to detect neutrinos from the sun based on Bruno Pontercovo and Luis Alvarez's idea for neutrino detection via $\nu_{e}$ capture on chlorine through the reaction ${ }^{37} \mathrm{Cl}+\nu_{e} \rightarrow{ }^{37} \mathrm{Ar}+e^{-}$. The first results from the experiment [11], located in the Homestake Mine in South Dakota, reported an upper bound for solar neutrinos that was a factor of two to three times below the predictions of the solar model developed by John Bahcall $[12,13]$. The observation of $\nu_{e}$ from the sun [14] confirmed that such fusion reactions were indeed occurring in the sun, although neutrinos were not observed at the predicted rate by the solar model. This became known as the solar neutrino problem. 
In 1968, Bruno Pontercovo suggested that such deficit in solar neutrinos could be due to neutrino oscillations [15]. Electron neutrinos, $\nu_{e}$, produced by fusion in the sun would oscillate to muon neutrinos, $\nu_{\mu}$, during their propagation to the Earth. During the following 30 years, different experiments with sensitivities to various $\mathrm{MeV}$ energy ranges found rate deficits of 0.3 to 0.7 compared to the solar model.

Low energy solar neutrinos from the primary pp fusion reactions were measured by the neutrino capture reaction $\nu_{e}+{ }^{71} G a \rightarrow{ }^{71} G e+e-$ in the SAGE, GALLEX and GNO experiments. Solar neutrino flux was measured in water Cherenkov detectors by Kamiokande and Super-Kamiokande experiments in Japan; and in heavy water in the Sudbury Neutrino Observatory (SNO) in Canada. In 2001, the latter experiment provided the definitive evidence of solar neutrino oscillations and therefore the existence of massive neutrinos [16].

\section{$2.2 \quad$ Neutrino Oscillations}

In 1957, Bruno Pontercovo, proposed for the first time the idea of transitions between neutrinos and antineutrinos through a process known as oscillations into sterile states. This was analogous in some way to the oscillations observed in the neutral kaon system. Gell-Mann and Pais [17] noted that the $K^{0}$ meson could turn into its antiparticle $\bar{K}^{0}$ through a second order weak interaction, and as a result the particles observed in the laboratory were not $K^{0}$ or $\bar{K}^{0}$, but instead they were some linear combination of the two.

For oscillations to occur among different neutrino types, it is required that the neutrino flavor states, $\nu_{\alpha}$, are different from the neutrino mass eigenstates, $\nu_{i}$. Quantum mechanics implies that if two of the observed neutrino flavor states $\left(\nu_{e}, \nu_{\mu}\right.$ and $\left.\nu_{\tau}\right)$ can spontaneously convert into the other, then neither of them is an eigenfunction of the Hamiltonian and therefore the true stationary states for the system must be some orthogonal linear superpositions of the neutrino mass eigenstates $\left(\nu_{1}, \nu_{2}\right.$ and $\left.\nu_{3}\right)$. 


\subsubsection{The $V_{P M N S}$ Mixing Matrix}

The neutrino flavor and mass eigenstates are related by the unitary matrix $V$ as follows,

$$
\nu_{\alpha}=\sum V_{\alpha i}^{*} \nu_{i}
$$

$\mathrm{V}$ is denoted as $V_{P M N S}$ in reference to its authors, Pontercovo-Maki-NakagawaSakata [18]. It is defined as,

$V_{P M N S}=\left[\begin{array}{ccc}c_{13} c_{12} & c_{13} s_{12} & s_{13} e^{-i \delta} \\ -s_{12} c_{23}-c_{12} s_{23} s_{13} e^{i \delta} & c_{12} c_{23}-s_{12} s_{23} s_{13} e^{i \delta} & s_{23} c_{13} \\ s_{12} s_{23}-c_{12} c_{23} s_{13} e^{i \delta} & -c_{12} s_{23}-s_{12} c_{23} s_{13} e^{i \delta} & c_{23} c_{13}\end{array}\right]\left[\begin{array}{ccc}1 & 0 & 0 \\ 0 & e^{\frac{i \phi_{21}}{2}} & 0 \\ 0 & 0 & e^{\frac{i \phi_{31}}{2}}\end{array}\right]$

where $c_{i j}=\cos \left[\theta_{i j}\right]$ and $s_{i j}=\sin \left[\theta_{i j}\right]$. For three neutrinos, the mixing matrix $V_{M N S}$ depends on three rotation angles $\theta_{23}, \theta_{13}$ and $\theta_{12}$ (where $0 \leq \theta_{i} \leq \pi / 2$ ) and depending on the neutrino nature, on one or three CP-violating phases $\delta, \phi_{2}$ and $\phi_{3}$ (where $0 \leq \delta, \phi_{i} \leq 2 \pi$ ). $\delta$ represents the so called Dirac phase in the case where the neutrino is of Dirac nature, which implies that massive neutrinos are distinct from their antiparticles; $\phi_{2}$ and $\phi_{3}$ represent the so called Majorana phase, under which assumption neutrinos are Majorana particles, implying that they are their own antiparticles.

\subsubsection{Two Neutrino Mixing}

\subsubsection{Survival Probability}

Assuming only two neutrino flavors, the mass eigenstates are given by [19],

$$
\begin{aligned}
& \nu_{1}=\cos \theta \nu_{\mu}-\sin \theta \nu_{e} \\
& \nu_{2}=\sin \theta \nu_{\mu}-\cos \theta \nu_{e}
\end{aligned}
$$

where the $\sin \theta$ and $\cos \theta \operatorname{coefficients~provide~an~elegant~normalization~in~terms~of~the~}$ mixing angle $\theta=\left[0, \frac{\pi}{2}\right]$. 
An important quantity to take into account when working with neutrino oscillations is the survival probability. This is the probability that a neutrino produced by the weak interaction as a flavor eigenstate $\nu_{\alpha}$ gets detected as the same flavor at a distance $L=c t$ from the source. For neutrinos in vacuum, and assuming only two neutrino flavors, it is given by

$$
P\left(\nu_{\alpha} \rightarrow \nu_{\alpha}\right)=1-\sin ^{2} 2 \theta \sin ^{2}\left(\frac{\Delta m^{2} L}{4 E}\right)
$$

where $L$ is the distance in $\mathrm{km}$ between the source and the detector, $E$ is the neutrino energy in $\mathrm{GeV}, \theta$ is the angle that describes the mixing between the flavor eigenstates and the mass eigenstates $\nu_{1}$ and $\nu_{2}$, and $\Delta m^{2}=m_{2}^{2}-m_{1}^{2}$ is the mass squared difference between the mass eigenvalues.

The appearance or transition probability, in which a new flavor is observed is given by

$$
P\left(\nu_{\alpha} \rightarrow \nu_{\beta}\right)=\sin ^{2} 2 \theta \sin ^{2}\left(\frac{\Delta m^{2} L}{4 E}\right)
$$

where $\alpha \neq \beta$.

These two probabilities are of extreme importance when analyzing data from neutrino appearance and disappearance experiments. By counting the number of neutrino interactions at both detector and source fundamental parameters such as $\theta$ or $\Delta m^{2}$ can be determined.

\subsubsection{Neutrino Oscillation Experiments}

The current experimental measurements of the neutrino mass splittings are shown in table 2.1.

\begin{tabular}{|c|c|c|}
\hline & Measured value $\left(\mathrm{eV}^{2}\right)$ & Experiment \\
\hline \hline$\triangle m_{21}^{2}$ & $7.5_{-0.20}^{+0.19} \times 10^{-5}$ & KamLAND \\
\hline$\triangle m_{32}^{2}$ & $2.32_{-0.08}^{+0.12} \times 10^{-3}$ & MINOS \\
\hline$\triangle m_{31}^{2}=\triangle m_{32}^{2}+\triangle m_{21}^{2}$ & $\pm 2.4 \times 10^{-3}$ & \\
\hline
\end{tabular}

Table 2.1: Neutrino mass splitting measurements at the $1 \sigma$ confidence level [21] 
Each of the mixing angles governs the oscillations of neutrinos from different sources. The angle $\theta_{23}$ governs the oscillations of atmospheric neutrinos, $\theta_{12}$ describes solar neutrino oscillations and the angle $\theta_{13}$ has been determined by reactor neutrino experiments at distances $L \sim 1 \mathrm{~km}$. Current values of the sin of these angles are listed in table 2.2 .

\begin{tabular}{|c|c|c|}
\hline & Measured value $\left(\mathrm{eV}^{2}\right)$ & Experiment \\
\hline \hline $\sin ^{2}\left(2 \theta_{12}\right)$ & $0.857_{-0.025}^{+0.023}$ & KamLAND, SNO \\
\hline $\sin ^{2}\left(2 \theta_{23}\right)$ & $>0.95$ & Super-Kamiokande \\
\hline $\sin ^{2}\left(2 \theta_{13}\right)$ & $0.092 \pm 0.016$ & CHOOZ, Daya Bay \\
\hline
\end{tabular}

Table 2.2: Neutrino mixing angle measurements at the $1 \sigma$ confidence level[21]

\subsubsection{Mass Hierarchy}

In the case of three neutrino mixing, there are three possible mass splittings,

$$
\triangle m_{21}^{2}=m_{2}^{2}-m_{1}^{2} \quad \triangle m_{32}^{2}=m_{3}^{2}-m_{2}^{2} \quad \triangle m_{31}^{2}=m_{3}^{2}-m_{1}^{2}
$$

where only two of them are independent, and are related as

$$
\triangle m_{31}^{2}=\triangle m_{21}^{2}+\triangle m_{32}^{2}
$$

Results from the SNO experiment in 2002 and the SuperK experiment in 1998 indicate that the smallest mass splitting is $\left|\triangle m_{21}^{2}\right|$ with $m_{1}<m_{2}$. However, these oscillation results are only sensitive to the difference of squares of the neutrino masses and don't provide an absolute value of the individual masses.

Having $m_{1}<m_{2}, \triangle m_{21}^{2}$ remains positive, there are two possible mass schemes,

- Normal Hierarchy If the mass eigenstate $m_{1}$ is the lightest one and

$$
m_{1}<m_{2}<m_{3}
$$


- Inverted Hierarchy If the mass eigenstate $m_{3}$ is the lightest one and

$$
m_{3}<m_{1}<m_{2}
$$

If the lightest eigenstate is $>0.1 \mathrm{eV}$, which in the order of magnitude of neutrino masses would be considered a heavy eigenstate, there would be another possible mass scheme,

- Quasi-Degenerate Hierarchy If the mass difference between the states is negligible compared to their total mass and

$$
m_{1} \simeq m_{2} \simeq m_{3} \simeq m_{\nu}
$$

\subsection{Massive Neutrinos in the Standard Model}

Within the Standard Model field theory, neutrinos are described as zero-mass particles. It becomes necessary to extend the SM Lagrangian formulation to implement neutrino masses in the model.

If neutrinos are massless, there are 19 free parameters in the Standard Model Lagrangian: three gauge couplings, six quark masses, three quark mixing angles and a $\mathrm{CP}$ - violating phase in the quark mixing matrix, the strong $\mathrm{CP}$ phase, three chargedlepton masses and the Higgs boson self-coupling and vacuum expectation value. If neutrinos have mass, there are at least seven more parameters: three neutrino masses, the corresponding three mixing angles, and one leptoninc CP-violating phase in the leptonic mixing matrix. If neutrinos are Majorana particles, there are also two other CP-violating phases.

\subsubsection{Dirac Mass}

Neutrinos are spin 1/2 particles, therefore they are described by the Dirac equation. The Dirac Lagrangian for a free neutrino field $\psi(x)$ is given by [26]

$$
\mathcal{L}(x)=\bar{\psi}(x)\left(i \gamma^{\mu} \partial_{\mu}-m_{D}\right) \psi(x)
$$


where the field adjoint is given by $\bar{\psi}(x)=\psi^{\dagger} \gamma^{0}$ and $m_{D}$ is the Dirac mass of the particle. The mass term of the Lagrangian is therefore,

$$
\mathcal{L}_{M}(x)=-m_{D} \bar{\psi} \psi
$$

By using the chirality projection operators

$$
P_{L}=\frac{1}{2}\left(1-\gamma_{5}\right) \quad P_{R}=\frac{1}{2}\left(1+\gamma_{5}\right)
$$

and defining $\psi_{L}$ and $\psi_{R}$ as the left and right handed components of the spinors,

$$
\psi_{L}=P_{L} \psi \quad \psi_{R}=P_{R} \psi
$$

we can write the mass term of the Dirac Lagrangian as follows

$$
\mathcal{L}_{M}=-m_{D}\left(\bar{\psi}_{L} \psi_{R}+\bar{\psi}_{R} \psi_{L}\right)
$$

The helicity of particles is defined as the projection of the spin vector on the momentum direction; that is, a right handed particle has spin in the direction of motion and helicity $H=+1$, a left handed particle has spin opposite to the direction of motion and its helicity is $H=-1$.

Equation (2.24) implies that the Dirac mass originates from the coupling of a neutrino left-handed spinor and a neutrino right-handed spinor. However, in weak interaction processes in the Standard Model, only left handed neutrinos $\nu_{L}$ and right handed antineutrinos $\bar{\nu}_{R}$ are observed constraining the neutrinos to be massless. In other words, if neutrinos were not massless they would travel at velocities smaller than the speed of light and their helicity would depend on the reference system; that is, it would be possible to "convert" a left handed neutrino into a right handed one by getting into a faster-moving reference frame and "reversing" the direction of motion.

\subsubsection{Majorana Mass}

The Dirac Lagrangian can be written using the two chiral components [26]

$$
\mathcal{L}=\left(\bar{\psi}_{L}+\bar{\psi}_{R}\right)\left(i \partial_{\mu} \gamma^{\mu}-m_{D}\right)\left(\psi_{L}+\psi_{R}\right)
$$




$$
=\bar{\psi}_{L}\left(i \partial_{\mu} \gamma^{\mu} \psi_{L}-m_{D} \psi_{R}\right)+\bar{\psi}_{R}\left(i \partial_{\mu} \gamma^{\mu} \psi_{R}-m_{D} \psi_{L}\right)
$$

Using the Euler-Lagrange equations one obtains,

$$
\begin{aligned}
& i \partial_{\mu} \gamma^{\mu} \psi_{L}=m_{D} \psi_{R} \\
& i \partial_{\mu} \gamma^{\mu} \psi_{R}=m_{D} \psi_{L}
\end{aligned}
$$

These two equations are known as the Weyl equations and are coupled by the mass constant term, $m_{D}$. For massless neutrinos these two equations decouple and then the neutrino can be described by a single chiral field (left-handed or righthanded). Majorana described massive neutrinos by using only a left-handed field, where the right-handed field is a function of the left-handed one (see eq(2.26)).

If we require the Majorana condition,

$$
\psi_{R}=C \bar{\psi}_{L}^{T}=\psi_{L}^{C}
$$

eqns (2.26) and (2.27) become equivalent. Moreover, the Majorana field becomes

$$
\psi=\psi_{L}+\psi_{R}=\psi_{L}+C \bar{\psi}_{L}^{T}=\psi_{L}+\psi_{L}^{C}
$$

and the charge conjugate results,

$$
\psi^{C}=\left(\psi_{L}+\psi_{L}^{C}\right)^{C}=\psi_{L}^{C}+\psi_{L}=\psi
$$

Therefore, the Majorana field is invariant under charge conjugation, implying the equality of particle and antiparticle. This field therefore only describes neutral fermions, and the neutrino under this description is the same as the antineutrino.

Describing the field only as a function of the left-handed (or right-handed) component, it is possible to incorporate to the Lagrangian the mass terms for both neutrino chiral states. The most general form of the Lagrangian mass term can be written as, 


$$
\mathcal{L}_{M}=-\frac{1}{2}\left(\bar{\psi}_{L}^{C} \bar{\psi}_{R}\right) M\left(\begin{array}{c}
\psi_{L} \\
\psi_{R}^{C}
\end{array}\right)+h . c
$$

where $\mathrm{M}$ is the most general neutrino mass matrix in the $\left(\psi_{L}, \psi_{R}^{C}\right)$ basis of lefthanded fields, and is given by

$$
M=\left(\begin{array}{ll}
m_{L} & m_{D} \\
m_{D} & m_{R}
\end{array}\right)
$$

where $m_{L}$ and $m_{R}$ are the Majorana mass terms for $\psi_{L}$ and $\psi_{R}^{C}$ respectively, and $m_{D}$ is the Dirac mass term.

\subsubsection{Absolute Neutrino Mass}

Observations of neutrino oscillations in solar, atmospheric, reactor and accelerator experiments are a direct evidence that neutrinos have non-zero rest mass but do not provide an absolute scale for their values.

The electron neutrino mass has been measured following Perri and Fermi's idea of studying the energy spectrum of the electrons emitted in beta decay. A non zero neutrino mass would cause a truncated spectrum at its endpoint. The common beta emitter is tritium, a heavy form of hydrogen in which two neutrons accompany the single proton in the nucleus. The studied reaction is

$$
{ }^{3} H \rightarrow{ }^{3} \mathrm{He}+e^{-}+\nu_{e}
$$

Collaborations from ITEP in Moscow, from the University of Zurich, from Los Alamos National Laboratory in New Mexico and from Mainz have placed restrictive upper bounds in the $e^{-}$neutrino mass using this technique and currently restrict the $\nu_{e}$ mass to be $<2.05 \mathrm{eV}[27]$.

The muon neutrino mass is determined by observing the decay of a charged pion at rest in the laboratory in the reaction,

$$
\pi^{+} \rightarrow \mu^{+}+\nu_{\mu}
$$


The PSI collaboration places an upper limit on the $\nu_{\mu}$ mass to be $<170 \mathrm{keV}$ at a confidence level of $90 \%$ [28] in accordance to the fact of being very much lighter than its corresponding charged lepton.

The experimental difficulties become greater for the $\nu_{\tau}$ due to the big mass of its lepton partner. The measurements of the tau neutrino mass come from observing the decay of the $\tau^{-}$into a $\nu_{\tau}$ in the interactions,

$$
\tau^{-} \rightarrow 2 \pi^{-} \pi+\nu_{\tau} \quad \tau^{-} \rightarrow 3 \pi^{-} 2 \pi+\nu_{\tau}
$$

The upper limit for the $\nu_{\tau}$ mass is set at $<18.2 \mathrm{MeV}$ by the ALEPH collaboration [29]. In 2000, the existence of the $\nu_{\tau}$ was confirmed by the DONUT (Direct Observation of the NU Tau) collaboration at Fermilab [20].

\subsection{Double Beta Decay}

Double beta decay is an extremely rare nuclear decay. It has been observed in 12 isotopes with lifetimes greater than $10^{18}$ years. It can occur via two principal modes: conversion of two neutrons to protons by the emission of two electrons and two electron antineutrinos, or conversion of two protons to neutrons by emission of two electron neutrinos and absorption of two electrons (electron capture). Furthermore, a neutrinoless mode is also possible to occur and constitutes a process of extreme importance for neutrino physics. Such mode violates lepton number conservation by two units and its observation would contribute towards the study of physics beyond the Standard Model. The standard interpretation of such neutrinoless double beta decay mode implies the existence of a so called light Majorana neutrino, whose absolute mass could be determined by the half-life measurement of the decay. This decay mode has never been observed in any isotope (although there exists a very controversial claim for ${ }^{76} \mathrm{Ge}$ ) and is expected to have lifetimes greater than $10^{23}$ years.

Double beta decay is a process by which a nucleus changes into an isobar with 
the spontaneous emission of two electrons and two antineutrinos, this is

$$
(A, Z) \rightarrow(A, Z+2)+2 e^{-}+2 \bar{\nu}_{e}
$$

This process conserves lepton number and occurs between two even-even nuclei in the cases when single beta decay of the parent nucleus is forbidden. Table [2.3] presents a list of isotopes for which double beta decay has been observed with their corresponding abundance and measured half life.

\begin{tabular}{|c|c|c|c|}
\hline Isotope & nat. Abundance $(\%)$ & $T_{1 / 2}^{2 \nu \beta \beta}\left(10^{21} y\right)$ & Experiments \\
\hline \hline${ }^{48} \mathrm{Ca}$ & 0.187 & $\left(4.4_{-0.4}^{+0.5} \pm 0.4\right) \times 10^{-2}$ & NEMO-3, CANDLES \\
\hline${ }^{76} \mathrm{Ge}$ & 7.8 & $1.74 \pm 0.01_{-0.16}^{+0.18}$ & GERDA, Majorana \\
\hline${ }^{82} \mathrm{Se}$ & 9.2 & $(9.6 \pm 0.3 \pm 1.0) \times 10^{-2}$ & NEMO-3, SuperNEMO \\
\hline${ }^{96} \mathrm{Zr}$ & 17.38 & $(2.35 \pm 0.14 \pm 0.16) \times 10^{-2}$ & NEMO-3 \\
\hline${ }^{100} \mathrm{Mo}$ & 9.6 & $0.69_{-0.08}^{+0.10} \pm 0.07$ & NEMO-3, MOON, AMoRe \\
\hline${ }^{116} \mathrm{Cd}$ & 7.6 & $(2.8 \pm 0.1 \pm 0.3) \times 10^{-2}$ & NEMO-3, COBRA \\
\hline${ }^{130} \mathrm{Te}$ & 34.5 & $0.7 \pm 0.09 \pm 0.11$ & NEMO-3, CUORICINO, CUORE \\
\hline${ }^{136} \mathrm{Xe}$ & 8.9 & $2.165 \pm 0.016 \pm 0.059$ & EXO-200, KamLAND Zen, NEXT \\
\hline${ }^{150} \mathrm{Nd}$ & 5.6 & $\left(9.11_{-0.22}^{+0.25} \pm 0.63\right) \times 10^{-3}$ & NEMO-3, SNO+ \\
\hline
\end{tabular}

Table 2.3: List of isotopes for which $2 \nu \beta \beta$ has been observed (at 68\% CL) [21].

\subsubsection{Neutrinoless Double Beta Decay}

In addition to the so called standard channel of double beta decay (equation (2.36)), there exists another possible transition, the neutrinoless double beta decay, $0 \nu \beta \beta$. It is a forbidden process in the Standard Model that admits violation of lepton number conservation by two units through the non-emission of the two daughter antineutrinos, this is

$$
(A, Z) \rightarrow(A, Z+2)+2 e^{-}
$$

If we think of this decay as a two-step process as described by the Racah sequence [26], we have 


$$
\begin{aligned}
(A, Z) \rightarrow & (A, Z+1)+e^{-}+\nu_{M} \\
& (A, Z+1)+e^{-}+\nu_{M} \rightarrow(A, Z+2)+2 e^{-}
\end{aligned}
$$

One can think of two possibilities for it to occur,

- In the first scenario, an antineutrino is emitted in the first step and must be absorbed as a left-handed neutrino in the second step. This implies that helicity is not conserved (and $m_{\nu}>0$ ) and furthermore that some transformation between $\nu$ and $\bar{\nu}$ must occur.

- In the second scenario, in both the first and second steps a right handed antineutrino is emitted and they annihilate. This implies that particle annihilation can occur between two antineutrinos or that the neutrino is its own antiparticle.

The most simple mechanism that explains this process introduces the idea of a massive virtual light Majorana neutrino $\left(\nu_{M}\right)$ exchange. This is, virtual neutrinos are produced in both steps and annihilate due to their Majorana nature (neutrinos are their own antiparticle). Furthermore, these virtual neutrinos are massive, resolving the helicity conservation issue. Feynman diagrams for both decay modes are shown in figure 2.1

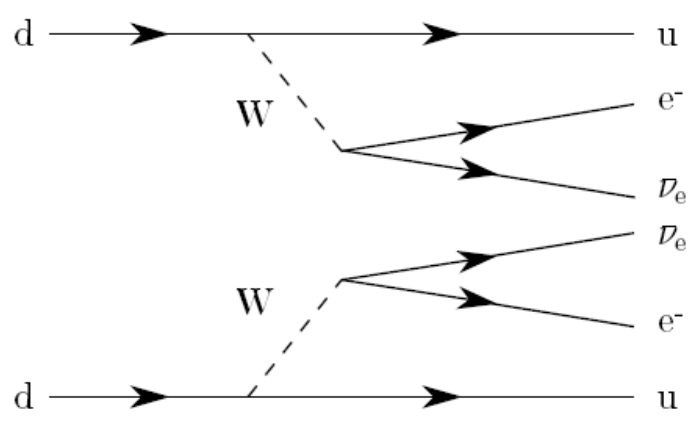

a) $2 v \beta \beta$

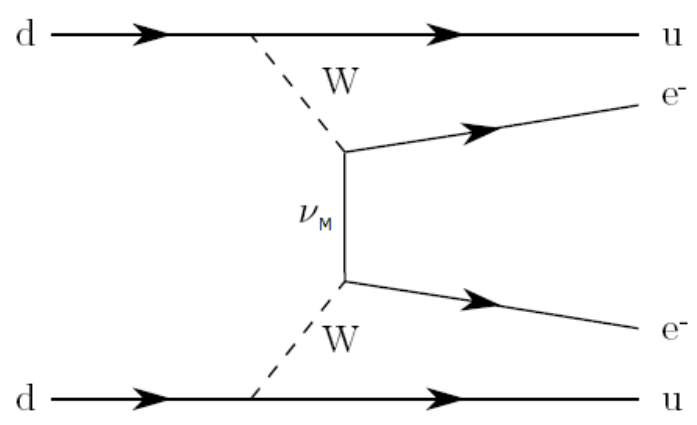

b) $0 v \beta \beta$

Figure 2.1: Feynman diagram for double beta decay modes. a) Double Beta Decay with emission of two antineutrinos (lepton number conserved). b) Neutrinoless Double Beta Decay (lepton number not conserved). 
Although most experimental efforts are currently focused on the detection of the $0 \nu \beta \beta$ decay mediated by a light Majorana neutrino exchange, this is not the only mechanism by which this process can occur. A third channel was proposed in 1980, which considers the emission together with the electrons of a Goldstone boson referred to as the Majoron $(\chi)$. Different models include the possibility of a $\chi$ carrying leptonic charge, in which case two Majorons would be emitted [30, 31, 32].

The observation of the neutrinoless double beta decay process is of great importance for neutrino physics beyond the Standard Model. It would address questions such as CP violation in the lepton sector and mass hierarchy. Furthermore, it would constitute a definitive evidence of the Majorana nature of the neutrinos and would establish an absolute scale of its mass.

\subsubsection{Decay Rates}

\subsubsection{Double Beta Decay Rate}

The decay rate for the $2 \nu \beta \beta$ process derived from the Fermi golden rule for a second order weak decay is given by [33]

$$
\frac{1}{T_{1 / 2}^{2 \nu \beta \beta}}=G^{2 \nu}(Q, Z)\left|M^{2 \nu}\right|^{2}
$$

where $T_{1 / 2}^{2 \nu \beta \beta}$ is the process half life, $M^{2 \nu}$ is the matrix element and $G^{2 \nu}$ is the phase space factor which depends on both the $Q$ value and the atomic number $Z$. The available energy that the particles have for the $2 \nu \beta \beta$ case is

$$
Q=m(A, Z)-m(A, Z+2)-2 m_{e}
$$

and the phase space factor is proportional to $G^{2 \nu} \propto Q^{7}$.

\subsubsection{Neutrinoless Double Beta Decay Rate}

The decay rate for the $0 \nu \beta \beta$ process derived from the Fermi golden rule for a second order weak decay is given by [33] 


$$
\frac{1}{T_{1 / 2}^{0 \nu \beta \beta}}=G^{0 \nu}(Q, Z)\left|M^{0 \nu}\right|^{2}\left(\frac{m_{\beta \beta}}{m_{e}}\right)^{2}
$$

where $T_{1 / 2}^{0 \nu \beta \beta}$ is the process half life, $M^{0 \nu}$ is the matrix element and $G^{0 \nu}$ is the phase space factor which depends on both the $Q$ value and the atomic number $Z$. The $Q$ value for the $0 \nu \beta \beta$ case is

$$
Q=E_{e_{1}}+E_{e_{2}}-2 m_{e}
$$

and the phase space factor is proportional to $G^{0 \nu} \propto Q^{5}$.

For the $0 \nu \beta \beta$ process a fundamental quantity appears, divided by the electron mass, $m_{e}$. This is a complex quantity known as the effective Majorana mass and defined as

$$
\left\langle m_{\beta \beta}\right\rangle=\sum_{k=1}^{3} V_{e k}^{2} m_{k}
$$

where $V$ is the PMNS matrix defined in section 2.2.1. Experiments on both double beta decay modes measure the half life of the decays. Phase space factors and nuclear matrix elements are computable, with the latter depending on the nuclear model.

The effective Majorana mass is related to the parameters of the PMNS matrix from the definition as follows

$$
\left|m_{\beta \beta}\right|=\left|m_{1} U_{e 1}^{2}+m_{2} U_{e 2}^{2}+m_{3} U_{e 3}^{2}\right|=\left|\left(m_{1} c_{12}^{2}+m_{2} s_{12}^{2}+e^{-i \phi_{21}}\right) c_{13}^{2}+m_{3} s_{13}^{2} e^{i\left(\phi_{31}-2 \delta\right)}\right|
$$

The three neutrino masses can be expressed in terms of the two measured values for the mass splittings, $\triangle m_{j k}^{2}$. Then, $\left|m_{\beta \beta}\right|$ is a function of the lightest neutrino mass, the Majorana and Dirac CP violation phases and of the type of neutrino hierarchy.

For the normal hierarchy, we have [21]

$$
\left|m_{\beta \beta}\right| \simeq\left|\sqrt{\triangle m_{21}^{2}} s_{12}^{2} c_{13}^{2}+\sqrt{\triangle m_{31}^{2}} s_{13}^{2} s_{13}^{2} e^{i\left(\phi_{31}-\phi_{21}-2 \delta\right)}\right|
$$


In the case of inverted hierarchy,

$$
\left|m_{\beta \beta}\right| \simeq \sqrt{\triangle m_{23}^{2}+m_{3}^{2}}\left(1-\sin ^{2}\left(2 \theta_{12}\right) \sin ^{2}\left(\frac{\phi_{21}}{2}\right)\right)^{1 / 2}
$$

Figure 2.2 shows the predicted $0 \nu \beta \beta$ effective Majorana mass as a function of the lightest neutrino mass. The quasi-degenerate spectrum corresponds to the region where both cases overlap.

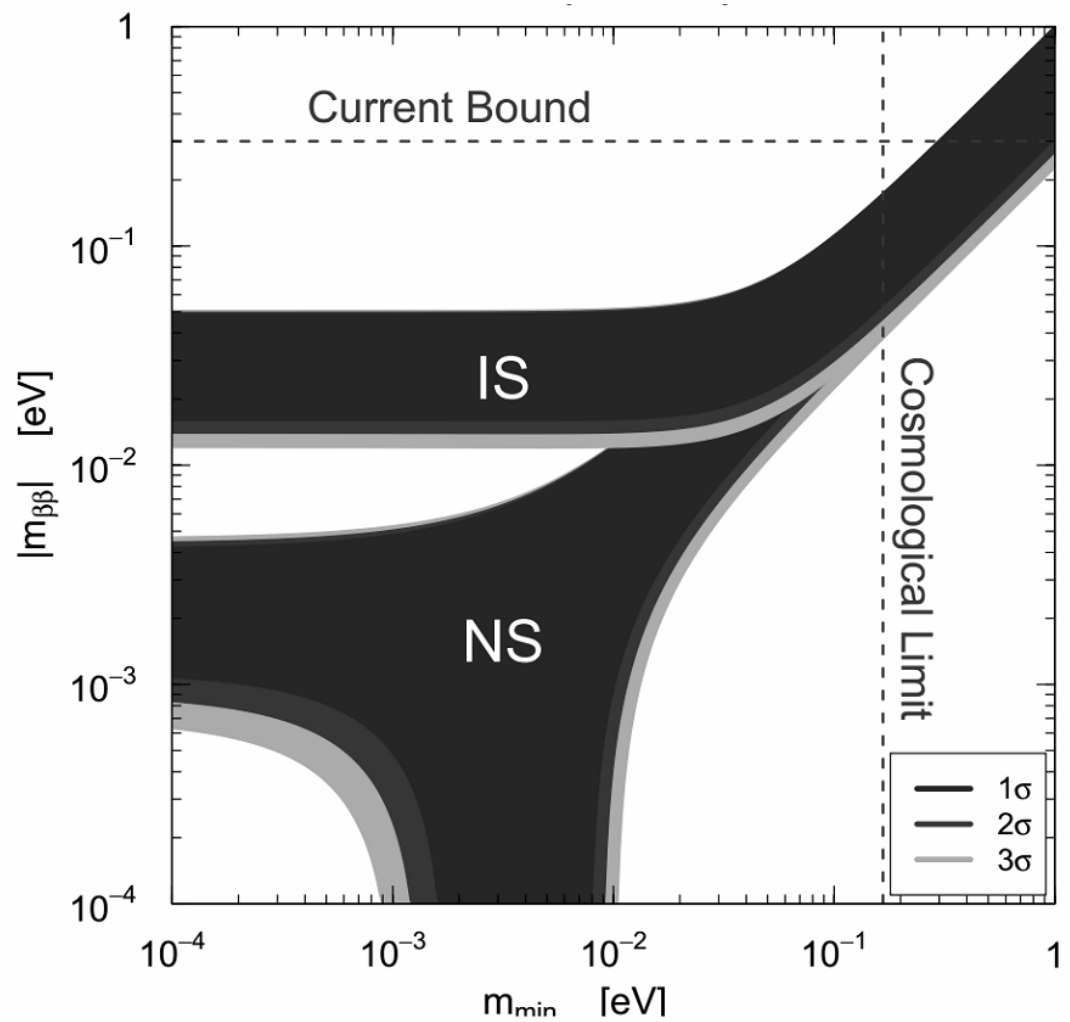

Figure 2.2: Effective Majorana mass as a function of the lightest neutrino mass in the normal and inverted hierarchies. Modified from [33]

Experiments on the search for neutrinoless double beta decay measure the half life of the decay. The sensitivity to perform such measurement is given by

$$
T_{1 / 2}^{0 \nu \beta \beta} \propto a \epsilon \sqrt{\frac{M t}{B(\Delta E)}}
$$

where a is the isotope enrichment, $\epsilon$ is the detector efficiency, $M$ is the mass, 
$t$ is the exposure time, $\Delta E$ is the energy resolution at the $\mathrm{Q}$ value and $B$ is the background rate at the energy region of interest.

\subsection{3 $0 \nu \beta \beta$ Decay Experiments}

Neutrinoless double beta decay observation require the operation of large detectors using sources of enriched isotopes, the least possible amount of radioactive backgrounds and excellent energy resolutions. In the $0 \nu \beta \beta$ process, the two electrons in the final state share all the transition energy $Q$, since the recoil of the daughter nucleus is negligible. The electron energy spectrum for this case would present a peak in the transition energy instead of a continuous energy spectrum like in the $2 \nu \beta \beta$ case. A diagram of the spectra of the sum of the electron kinetic energies $K_{e}(Q$ is the endpoint) for the $2 \nu \beta \beta$ and $0 \nu \beta \beta$ decays is shown in figure 2.3 . Therefore, in order to discriminate between the two decay modes, a high resolution measurement of the electron energy is required.

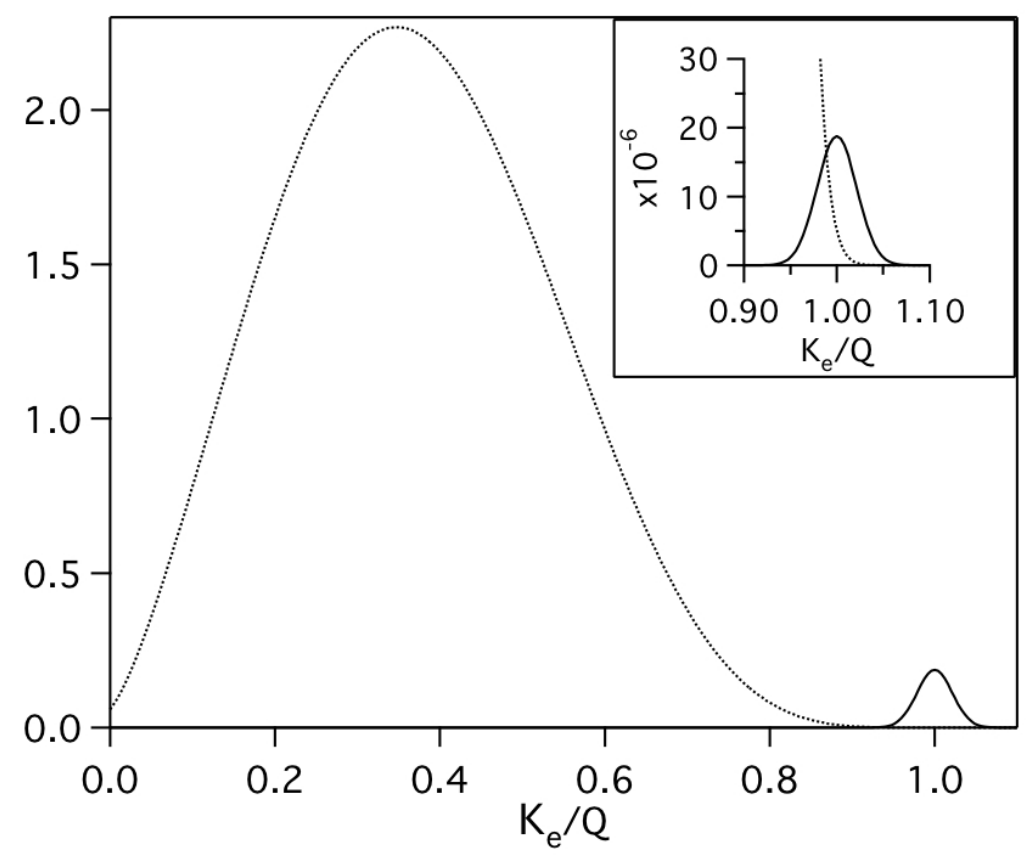

Figure 2.3: Spectra of the sum of the electron kinetic energies $K_{e}$ (endpoint $Q$ ) for the $2 \nu \beta \beta$ (dotted curve normalized to 1 ) and $0 \nu \beta \beta$ (solid curve normalized to $10^{-6}$ ). Taken from [67]. 
There are currently a number of experiments in various stages of research, development and operation searching for $0 \nu \beta \beta$ in different isotopes. EXO-200 $\left({ }^{136} X e\right)$, KamLAND-Zen $\left({ }^{136} \mathrm{Xe}\right)$ and GERDA $\left({ }^{76} \mathrm{Ge}\right)$ are currently taking data. CUORE $\left({ }^{130} \mathrm{Te}\right)$, Majorana $\left({ }^{76} \mathrm{Ge}\right), \mathrm{SNO}+\left({ }^{150} \mathrm{Nd}\right)$ are currently in construction. SuperNEMO $\left({ }^{82} \mathrm{Se}\right)$, NEXT $\left({ }^{136} \mathrm{Xe}\right)$, COBRA $\left({ }^{116} \mathrm{Cd}\right)$, MOON $\left({ }^{100} \mathrm{Mo}\right)$ and CANDLES $\left({ }^{48} \mathrm{Ca}\right)$ are in research phase.

Table 2.4 presents the most updated results of the lower bounds on the $T_{1 / 2}^{0 \nu \beta \beta}$ and corresponding upper bounds on the effective neutrino mass $m_{\beta \beta}$ from experiments on the search of neutrinoless double beta decay. There has been one positive claim for the observation of neutrinoless double beta decay on ${ }^{76} \mathrm{Ge}$ by Klapdor-Kleingrothaus et al. [8] reporting a half life of $T_{1 / 2}^{0 \nu \beta \beta}=1.2 \times 10^{25} \mathrm{y}$ at the $95 \%$ confidence level. Extrapolating such value for the case of ${ }^{136} \mathrm{Xe}$, it corresponds to a half life between $2 \times 10^{24}$ to $2 \times 10^{25}$ years for different matrix elements. Note from 2.4 that EXO-200 and Kamland-Zen measurements are consistent, ruling out for most matrix elements the positive observation of ${ }^{76} \mathrm{Ge}$ neutrinoless double beta decay claimed by KlapdorKleingrothaus.

\begin{tabular}{|c|c|c|c|c|}
\hline Isotope & CL & $T_{1 / 2}^{0 \nu \beta \beta}\left(10^{21} y\right)$ & $\left\langle m_{\beta \beta}\right\rangle(e V)$ & Claim \\
\hline${ }^{48} \mathrm{Ca}$ & 90 & $>13$ & $<3.5-22$ & $\mathrm{CaF}_{2}$ scintillator \\
\hline${ }^{76} \mathrm{Ge}$ & 90 & $>21000$ & $0.32 \pm 0.03$ & GERDA, Klapdor- Kleingrothaus \\
\hline${ }^{82} \mathrm{Se}$ & 90 & $>100$ & $<0.89-2.43$ & NEMO-3 \\
\hline${ }^{96} \mathrm{Zr}$ & 90 & $>9.2$ & $<7.2-19.5$ & NEMO-3 \\
\hline${ }^{100} \mathrm{Mo}$ & 90 & $>1100$ & $<0.45-0.93$ & NEMO-3 \\
\hline${ }^{116} \mathrm{Cd}$ & 90 & $>16$ & $<1.5-1.7$ & NEMO-3 \\
\hline${ }^{130} \mathrm{Te}$ & 90 & $>940$ & $<0.19-0.68$ & CUORICINO \\
\hline${ }^{136} X e$ & 90 & $\begin{array}{l}>16000 \\
>19000\end{array}$ & $\begin{array}{l}<0.14-0.38 \\
<0.12-0.25\end{array}$ & $\begin{array}{c}E X O-200 \\
\text { KamLAND-Zen }\end{array}$ \\
\hline${ }^{150} \mathrm{Nd}$ & 90 & $>18.0$ & $<4.0-6.8$ & NEMO-3 \\
\hline
\end{tabular}

Table 2.4: Sensitivities to $T_{1 / 2}^{0 \nu \beta \beta}$ and $\left\langle m_{\beta \beta}\right\rangle$ (for the range in nuclear matrix element calculations) reached by experiments searching for $0 \nu \beta \beta[21]$.

There are three current operating experiments in the search for neutrinoless double beta decay. GERDA is located at the Gran Sasso Laboratory in Italy and started physics data taking in November 2011. It consists of an array of high purity Ge de- 
tectors immersed in high purity liquid argon, enclosed by a water shield. The Ge detectors constitute a total mass of $17.66 \mathrm{~kg}$ of germanium enriched to $86 \%$ in ${ }^{76} \mathrm{Ge}$ [22]. KamLAND-Zen is located at the Kamioka mine in Japan and started operation in September 2011. The setup consists of a $\sim 3 \mathrm{~m}$ radius inner balloon filled with 13 tonnes of liquid scintillator loaded with $330 \mathrm{~kg}$ of Xe gas enriched to $91 \%$ in ${ }^{136} \mathrm{Xe}$, surrounded by a sphere of liquid scintillator submerged inside a $9 \mathrm{~m}$ radius stainless-steel sphere containing 3000 tonnes of oil. Scintillation light is detected by PMTs mounted on the stainless steel tank wall [23]. EXO-200 resides at the Waste Isolation Pilot Plant in Carlsbad, New Mexico and started low background data taking in May 2011. It consists of a liquid time projection chamber filled with $200 \mathrm{~kg}$ of Xenon enriched to $80.6 \%$ in ${ }^{136} \mathrm{Xe}$. The detector measures energy from ionization and scintillation signals and constitutes a prototype for a 1-10 tonne scale experiment [38].

Within the next few years two other experiments will come to operation and are currently in a construction phase. CUORE will have $988 \mathrm{TeO}_{2}$ crystals operated as bolometers providing a total of $203 \mathrm{~kg}$ of tellerium enriched in the isotope ${ }^{130} \mathrm{Te}$. The bolometers operate at temperatures between $8-10 \mathrm{mK}$, where the heat capacity is small. The energy deposited in the array is measured from the temperature rise in the detector. CUORE will operate at Gran Sasso Laboratory and will start data taking in 2014 [24]. A MAJORANA prototype is being constructed at the Sanford Underground Research Facility (SURF) at the old Homestake gold mine in Lead, South Dakota. The setup consists of $40 \mathrm{~kg}$ of Ge p-type detectors enriched to $86 \%$ in ${ }^{76} \mathrm{Ge}$, enclosed within two separate copper cryostats contained within a shield of copper, lead and plastic. It will be commissioned by the end of 2013 and will join forces with GERDA to build an improved detector based on the results obtained from this prototype and the second phase of Gerda [25].

In order to probe the allowed region for the inverted neutrino mass hierarchy, experiments need sensitivities to half lives on the order of $>10^{27}$ years, corresponding to effective neutrino masses of $\sim 50 \mathrm{meV}$, depending on the nuclear matrix elements. Next phases of experiments such as nEXO, KamLAND-Zen, CUORE, Majorana and Gerda Phase II are being designed to reach such sensitivities. 


\section{Chapter 3}

\section{The Enriched Xenon Observatory}

As previously discussed, the observation of $0 \nu \beta \beta$ decay will help answer fundamental questions about the nature of the neutrino (Dirac or Majorana), about the mechanism by which the neutrino mass is generated according to theories beyond the standard model, and about the $\nu$ absolute mass. Experiments all over the world are involved in this search that will complement the currently known properties of the neutrinos established by beta decay measurements, cosmological constraints and neutrino oscillation data.

The advantages and disadvantages of the current experiments in operation and under development reside in whether they can achieve good sensitivities for the detection of neutrinoless double beta decay. Experiments such as EXO-200, KamLAND-Zen and $\mathrm{SNO}+$, despite not having excellent energy resolutions, take advantage of the isotope mass to build tonne scale detectors that would improve the detection sensitivity. In the case of the first two, their greatest advantage is the possibility to perform a measurement in a background free environment by the identification of the daughter product of the decay $\left({ }^{136} \mathrm{Ba}\right)$. Mass scaling of the detector for the case of CUORE, GERDA or MAJORANA is not as feasible as for other cases. Their major strength resides in the excellent energy resolution that their detector technology (Ge detectors and bolometers) could achieve. Precision tracking in the detector as planned for NEXT and SuperNEMO would be an ideal approach to achieve very high detection efficiencies and good energy resolution. 
Building a detector for the search of such a rare event requires a series of considerations that exploit the strengths of the isotope to study and the available technology. Section 3.1 presents some experimental factors to be taken into account in the detector design for the observation of $0 \nu \beta \beta$. The two prototypes (liquid and gas) built by the EXO experiment to perform the energy measurements of the decay are described in Section 3.2. And finally, the principles for barium identification using laser induced fluorescence and the barium tagging concept for a gaseous xenon TPC are outlined in Section 3.3.

\subsection{Experimental Criteria}

Neutrinoless double beta decay can be identified by its distinguishing sum energy peak at its $\mathrm{Q}$ value. To reach the best sensitivity to $\left\langle m_{\beta \beta}\right\rangle$, it is necessary to build a detector for which the count rate of the decay is maximized by reducing the amount of background events. Background suppression around the region of interest plays one of the most important and challenging aspects of the detector design, construction and performance.

For experiments where source and shielding materials have long lived isotopes, cosmogenic activities constitute a significant background. Fabricating, storing and placing equipment in deep underground locations with appropriate shielding from muons and neutrons help reduce this effect.

Another important background comes from natural activity of isotopes in the surroundings of the detector. It is necessary that both source and detector are very low in impurities and that both are shielded from the environment with a radio pure material. This will minimize the amount of high energy gamma rays coming from the ${ }^{238} \mathrm{U}$ and ${ }^{232} \mathrm{Th}$ chains occurring in the rock and detector materials. Two of the most relevant products of these decay chains are ${ }^{214} \mathrm{Bi}$ and ${ }^{208} \mathrm{Tl}$. A photon of $2614.5 \mathrm{keV}$ (100\% intensity) is emitted in the ${ }^{208} \mathrm{Tl}$ decay along with the $\beta$ particle and other photons; ${ }^{214} \mathrm{Bi}$ has two $\beta$ decays with endpoints at $2663 \mathrm{keV}$ (1.7\% intensity) and $3272 \mathrm{keV}$ (18.2\% intensity), and a $\gamma$ ray of $2447.8 \mathrm{keV}$ (1.5\% intensity). All these decay channels can lead to secondary electrons via Compton and photoelectric 
interactions.

Collection of ionization (and scintillation) signals and segmentation of the detector help in the achievement of the best energy and position resolution. This is taken into account to improve the signal to background ratio around the $\mathrm{Q}$ value, avoiding the $2 \nu \beta \beta$ signal to extend to the region of interest becoming a background itself.

To optimize the design of a $0 \nu \beta \beta$ experiment, according to equation (2.47), large amounts of source mass are required. To reach the $50 \mathrm{meV}$ region for the absolute mass scale, approximately a tonne of the enriched isotope of choice is required. Since background effects increase with the volume, it is ideal to minimize the detector size, and furthermore, employ the studied isotope both as source and detection medium.

Any radioactive isotope with a $\mathrm{Q}$ value greater than the neutrinoless double beta decay endpoint may become a potential background. In the selection of the isotope to study, it is optimal to choose a $\beta \beta$ candidate with the largest possible $\mathrm{Q}$ value, as the number of radioactive isotopes decreases with increasing Q. Although having a high $\mathrm{Q}$ value implies having higher decay rates for $0 \nu \beta \beta$, it also gives rise to other complications related to the decay rate of the $2 \nu \beta \beta$ process. As observed from equations (2.39) and (2.41), the decay rate for the $2 \nu \beta \beta$ decay depends on $Q^{7}$, while the decay rate for the $0 \nu \beta \beta$ decay is proportional to $Q^{5}$. In other words, as the $\mathrm{Q}$ value increases the rate of the two neutrino double beta decay could overtake the neutrinoless double beta decay becoming a potential and irreducible background for the measurement of the latter.

One of the isotopes of more interest is ${ }^{76} G e$, for which a claim for the observation of $0 \nu \beta \beta$ has been made by by Klapdor- Kleingrothaus [31]. Experiments like GERDA and Majorana are in the process of verifying this claim. ${ }^{136} \mathrm{Xe}$ is an interesting isotope to study. It has a $\mathrm{Q}$ value equal to $2457.83 \pm 0.37 \mathrm{keV}$ in a region relatively free from naturally occurring radioactive backgrounds. Its natural abundance is $9 \%$ and isotopic enrichment can be easily performed by centrifugation. Furthermore, it can be re-purified into the detector, it doesn't have long lived isotopes, it is self shielding, it represents a good ionization detecting medium and responds to the passage of 
particles by a $<100$ ns scintillation light. Therefore, ${ }^{136} \mathrm{Xe}$ can be used as the detection medium in either a noble liquid or gas time projection chamber (TPC). Another advantage of using ${ }^{136} \mathrm{Xe}$ is that its double beta decay produces a ${ }^{136} \mathrm{Ba}$ nucleus that can be identified using high resolution spectroscopy. EXO-200 and KamLAND-Zen experiments are currently studying $0 \nu \beta \beta$ in ${ }^{136} \mathrm{Xe}$. Their consistent measurements have ruled out for most matrix elements the positive observation of ${ }^{76} \mathrm{Ge}$ neutrinoless double beta decay claimed by Klapdor- Kleingrothaus.

\subsection{EXO Experiment}

There are two phases under study by the EXO collaboration for the observation of neutrinoless double beta decay in ${ }^{136} \mathrm{Xe}$. The first one is EXO-200, a $200 \mathrm{~kg}$ liquid Xe time projection chamber currently operating and taking physics data at the Waste Isolation Pilot Plant (WIPP) in Carlsbad, NM; the second one is XEP (Xenon Electroluminescence Prototype), a $1 \mathrm{~m}^{3}$ gas TPC to be operated at pressures between 1 and 10 bar currently in a research and development stage at Carleton University in Ottawa, ON. For both phases, techniques for the tagging of the ${ }^{136} \mathrm{Ba}$ daughter of the decay are being studied by the collaboration. Both phases will be described in the following subsections as well as the Barium tagging concept for the gaseous phase currently under study by collaborators at different institutions.

\subsubsection{EXO Liquid Xenon Prototype (EXO-200)}

One of the main advantages of using liquid Xe is the fact that the detector can be very compact. At atmospheric pressure and room temperature, $200 \mathrm{~kg}$ of xenon occupy $0.07 \mathrm{~m}^{3}$ in its liquid phase and $38 \mathrm{~m}^{3}$ in its gas phase. Moreover, there is no need to pressurize the xenon vessel and electron diffusion is almost negligible. The disadvantages are that a liquid phase detector requires cryostat cooling and has limited track reconstruction. EXO-200 uses liquid xenon as both source and detector in a homogeneous TPC, taking advantage of its self-shielding properties and the possibility of purification before and during its use. It was built as a prototype for a future larger detector in the multi tonne scale. Figure 3.1 shows the schematics of the EXO-200 TPC. 


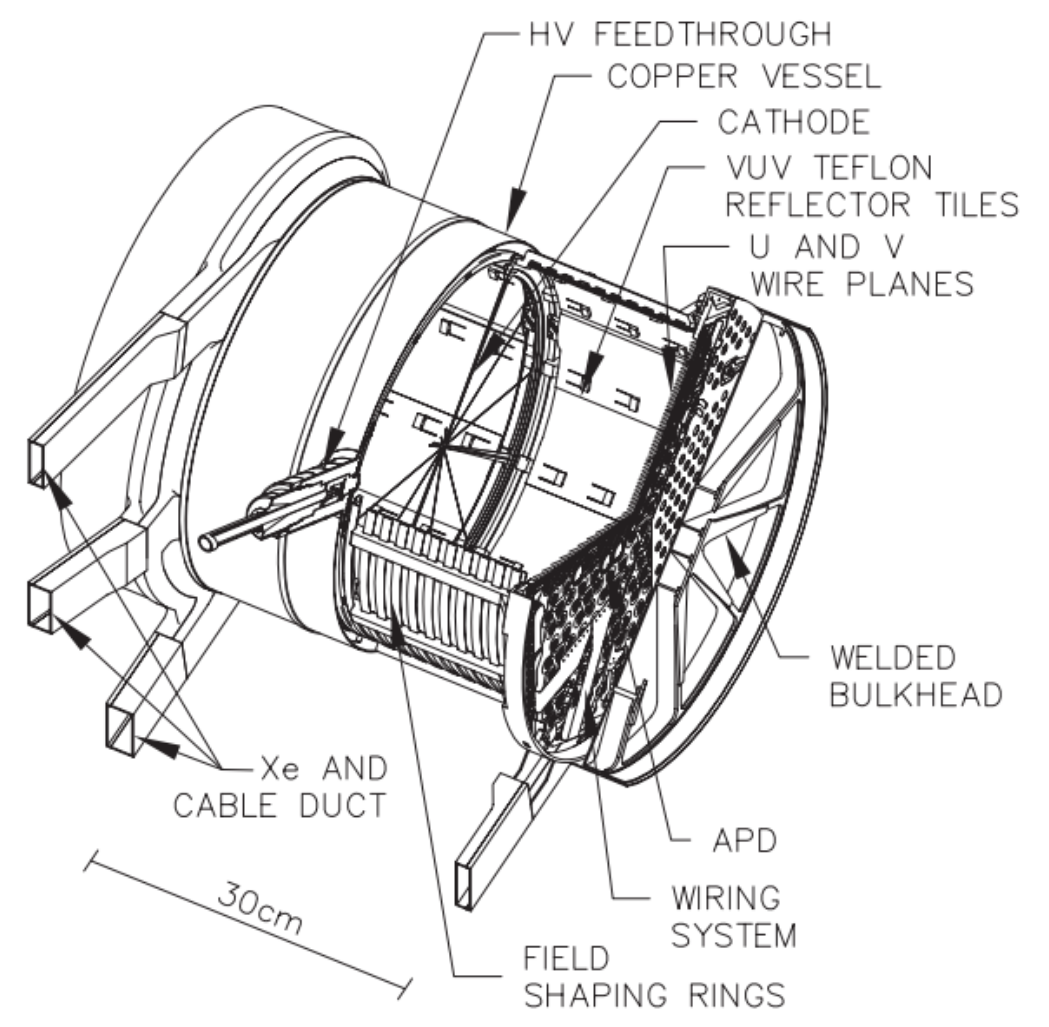

Figure 3.1: Schematics of EXO 200 TPC. Adapted from [38]

The detector consists of a cylindrical double time projection chamber (TPC) of $\sim 40 \mathrm{~cm}$ length, separated with a cathode grid held at a negative potential. It is filled with liquid xenon enriched to $80.6 \%$ in the isotope ${ }^{136} \mathrm{Xe}$ and operates at a temperature of $167 \mathrm{~K}$ and a pressure of $147 \mathrm{kPa}$. The signal readout is performed by crossed wire planes ( $\mathrm{U}$ and $\mathrm{V}$ ) held at ground potential and avalanche photodiodes at each side of the detector. Reflective PTFE Teflon tiles are placed around the field cage to improve light collection efficiency. The charge collection (U) and shielding (V) wire planes are biased to ensure full electron transparency. The $\mathrm{U}$ and $\mathrm{V}$ wire planes are spaced $6 \mathrm{~mm}$ from each other and are oriented $60^{\circ}$ from each other. Of the 200 $\mathrm{kg}$ of enriched xenon available, $175 \mathrm{~kg}$ is in liquid form with $110 \mathrm{~kg}$ available in the active volume of the detector; the remaining mass of Xe is in gaseous form around the handling system. A full description of the detector design and construction can be found in [38]. 
Energy deposited in the liquid xenon from physics events produces both an ionization and a scintillation signal. The detector records the former by drifting the secondary electrons in the uniform electric field towards the crossed wire planes, and the charge induced and collected by them provides the energy of the ionizing particles. The latter, a $178 \mathrm{~nm}$ ultraviolet (VUV) scintillation light generated by the excitation of Xe atoms by the primary electrons [39], is picked up by large area avalanche photodiodes (LAAPD). The arrival time difference between the scintillation light and the first ionization collection determines the electron drift time and provides the $\mathrm{z}$ coordinate of the event. The $\mathrm{U}$ and $\mathrm{V}$ wires determine the respective radial and angular coordinates allowing a three dimensional reconstruction of the event location.

Calibrations with radioactive gamma ray sources are frequently performed by placing sources inside the detector. This is done by inserting the source of choice $\left({ }^{137} \mathrm{Cs}\right.$, ${ }^{60} \mathrm{Co}$, and ${ }^{228} \mathrm{Th}$ ) in a copper guide tube that goes around the outside of the LXe vessel in the HFE-7000 volume, which is the coolant responsible of the thermal uniformity of the system. The sources can be placed at different determined locations around the detector.

Barium tagging is not included in the EXO-200 prototype. Collaborators are investigating possible techniques for the barium extraction and identification using the liquid phase. Some of the concepts include: in-situ tagging by bringing lasers into the TPC and collecting fluorescence light; capture and extraction of the $\mathrm{Ba}^{++}$ ion from the xenon vessel to a radio frequency linear Paul trap for observation of fluorescence in vacuum; and freezing of the $\mathrm{Ba}^{++}$ion in a solid xenon probe and detecting fluorescence while it is in the TPC. All these concepts are currently in a research and development stage. 


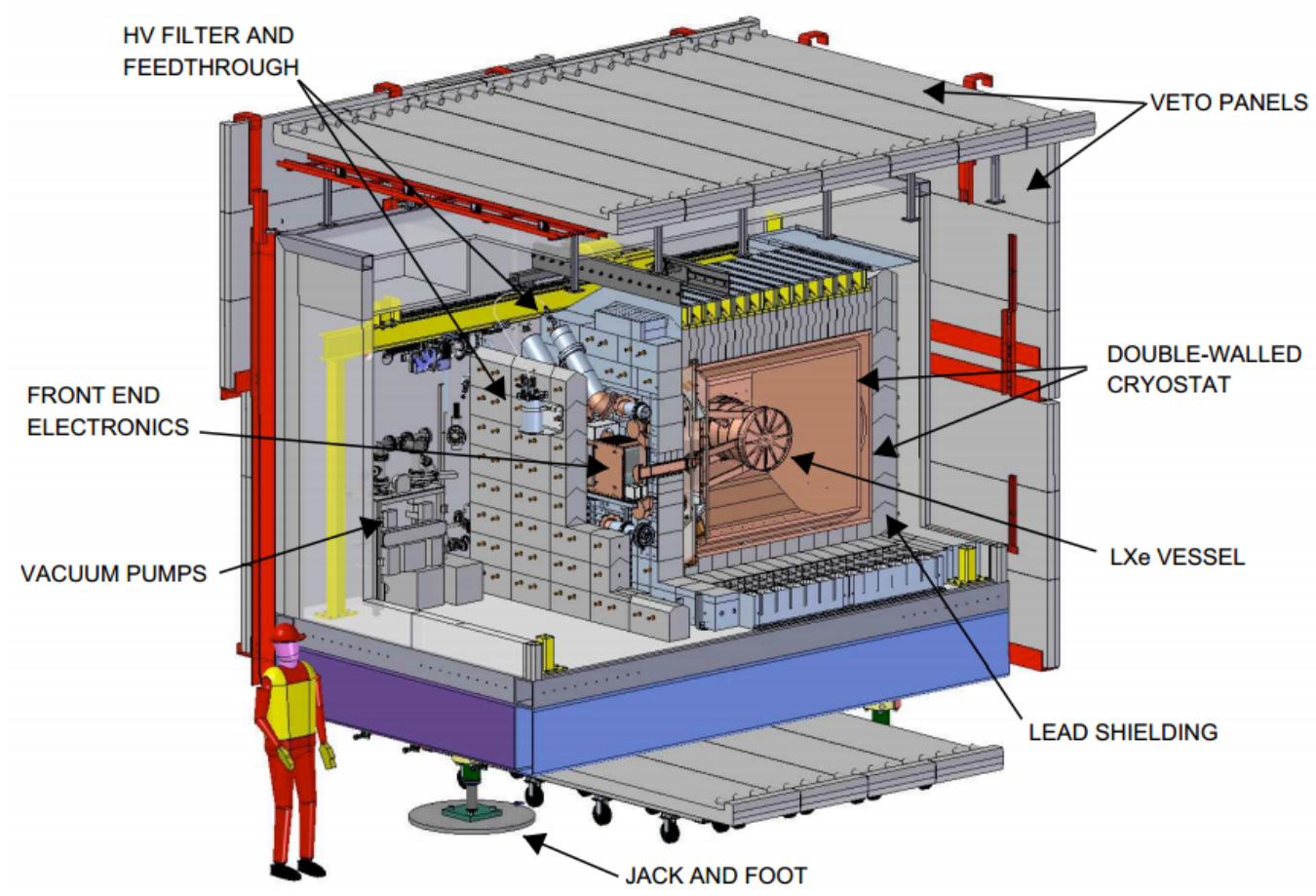

Figure 3.2: Schematics of the EXO 200 setup. Adapted from [38]

Figure 3.2 shows the schematics of the entire detector complex. The TPC sits inside a double-walled copper cryostat, which is filled with HFE7000 fluid and surrounded by a lead wall for radioactive shielding. There are two primary vacuum systems for the setup, one handles the cryostat vessel and the other the insulation of the refrigeration system. Small regions around the flanges, feedthrough plates and refrigeration line junctions are also pumped. Copper feedthroughs are welded onto the cryostat for electrical connections to the TPC and for the supply and return lines of the xenon and the HFE .

The whole experiment is housed inside a class 1000 clean room, surrounded by cosmic ray veto counters, operating at a depth of $1585 \mathrm{~m}$ water equivalent [37] at the Waste Isolation Pilot Plant (WIPP) in Carlsbad, NM, designed to achieve a sensitivity of the neutrino mass on the order of $\sim 100 \mathrm{meV}$. It started low-background data taking in May 2011 and in November 2011 reported the first observation of $2 \nu \beta \beta$ in ${ }^{136} \mathrm{Xe}$ [34], this value was confirmed by the KamLAND-Zen collaboration in 2012 
and the most recent reported values are $T_{1 / 2}^{2 \nu \beta \beta}=2.165 \pm 0.016 \pm 0.059 \times 10^{21} y[35]$ and $T_{1 / 2}^{2 \nu \beta \beta}=2.38 \pm 0.02 \pm 0.14 \times 10^{21} y$ [36] respectively. EXO-200 is the prototype for a multi tonne detector currently in a design stage, which will reach Majorana mass sensitivity in the inverted hierarchy region below $10 \mathrm{meV}$.

\subsubsection{EXO Gaseous Xenon Prototype}

A gaseous xenon TPC appears very attractive for a variety of reasons: it would achieve better energy resolution than a liquid TPC [41], it can be operated at room temperature, it offers the possibility of electron tracking and it would facilitate the extraction of barium ions from the vessel. From the background rejection point of view, a gas TPC is the ideal setup for the observation of neutrinoless double beta decay. However, this phase requires a larger volume detector, extensive shielding, and a low background pressurized vessel. A high pressure gaseous xenon TPC has been operated before by the Gotthard experiment [42] and is intended to be used by the NEXT experiment collaboration for the neutrinoless double beta decay observation of ${ }^{136} \mathrm{Xe}$. NEXT is planned to use $>100 \mathrm{~kg}$ of Xe enriched to $90 \%$ in ${ }^{136} \mathrm{Xe}$. The detector will consist of a gas TPC operating at pressures $\sim 15$ bar. It will detect scintillation light and will perform electron tracking for reconstruction of events. Its commissioning will start in 2014 at the Laboratorio Subterraneo de Canfranc in Spain (LSC) [43].

The tracking capabilities of the TPC provide a reliable way to measure and discriminate background events in the detector as every physics process will have a distinctive topological signature. For instance, a double beta event will produce an ionization track of $\sim 30 \mathrm{~cm}$ at 10 bar with larger depositions in both ends of the TPC corresponding to the two stopping electrons. A single electron, on the other hand, will only produce one such deposition.

Collaborators at Carleton University, in Ottawa, Canada are currently constructing and developing a $1 \mathrm{~m}^{3}$ gaseous xenon TPC to operate at pressures between 1 and 10 bar. This prototype measures both scintillation and ionization signals, as well as electroluminescence to amplify the ionization signal and improve energy res- 
olution. A schematic drawing of the so called Xenon Electroluminescence Prototype (XEP) is shown in figure 3.3.

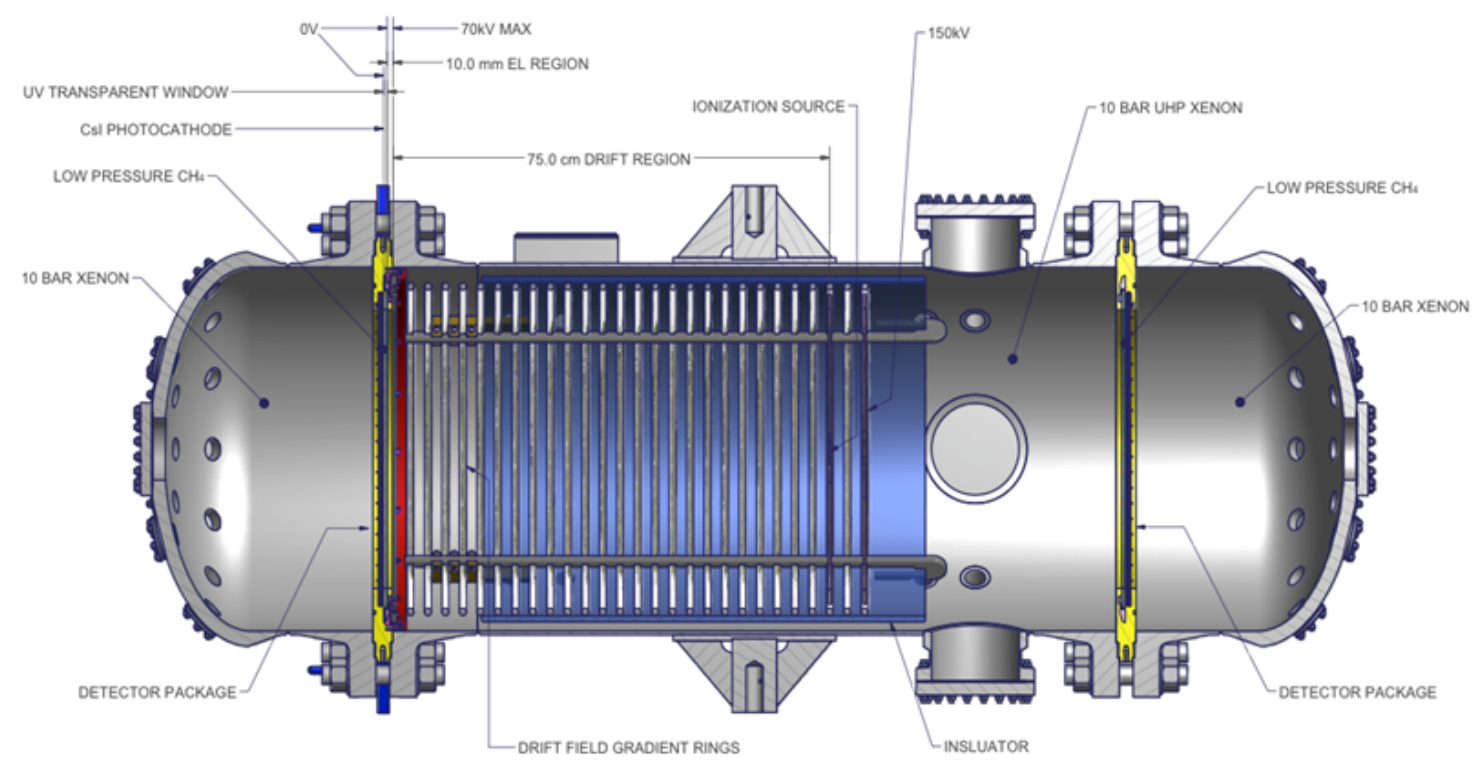

Figure 3.3: Schematic of gaseous xenon prototype detector. Adapted from [40]

The detector consists of 27 electrode rings that compose a $75 \mathrm{~cm}$ drift region that can reach up to $1000 \mathrm{~V} / \mathrm{cm}$. The electroluminescence region at one side of the vessel is $1 \mathrm{~cm}$ wide and reaches up to $70000 \mathrm{~V} / \mathrm{cm}$. The light collection is performed by two Caesium iodide photodetectors located at each side of the drift region. A reflective Teflon cylinder surrounds the field shaping rings increasing the light collection efficiency.

When a physics event occurs, it generates an ionization signal inside the detector. Electroluminescence light from the secondary electrons is measured and these are drifted into the high electric field region. Electrons gain energy and excite the xenon causing it to radiate ultraviolet light which is then picked up by the two cesium iodide photodetectors. One of them records the spatial distribution of the secondary electrons, while the other (at the other extreme of the TPC) records a smooth light distribution. This provides a primary track reconstruction and an energy measurement of the ionizing event. 
XEP is planned to operate with $10 \mathrm{~kg}$ of non-enriched pure xenon at pressures between 1 and 10 bar. The detector construction did not follow extreme low background specifications and it is being operated at surface level. Therefore, it won't be suitable for a measurement of the $0 \nu \beta \beta$ or $2 \nu \beta \beta$ rate of ${ }^{136} X e$. The main purpose of the detector is to test the electroluminescence concept in xenon and establish the conditions needed to operate and extract energy information from it. Once in operation it will be useful to proof the barium tagging concept described in the next section.

\subsection{Barium Tagging in a Gaseous Xe TPC}

In order to scale up a detector to operate with large masses of the isotope of interest it is important to consider the scaling of the backgrounds and the fact that the measurement can be completely dominated by them. Kinematic reconstruction of $0 \nu \beta \beta$ events in coincidence with the detection of $\mathrm{Ba}$ ions would constitute a backgroundfree technique for the measurement of $0 \nu \beta \beta$ in ${ }^{136} \mathrm{Xe}$. Direct tagging of the final state Ba ion using optical spectroscopy was first suggested by M.K.Moe in 1991 [45] and developed into a conceptual design for a gas xenon detector by M. Danilov et al in 2000 [46]. This section will describe the basics for laser detection of single atoms and how it would be possible to achieve using a gaseous xenon TPC. Such barium tagging concept is currently under development by EXO collaborators at Stanford and Carleton University.

\subsubsection{Single Atom Spectroscopy}

The atomic level structure of ${ }^{136} \mathrm{Ba}^{+}$(figure 3.4) is similar to that of an alkali atom. It has a single valence electron in the $s$ shell and can be treated as a single electron atom within the theory of quantum mechanics. Single $\mathrm{Ba}^{+}$ions were first observed using a RF quadrupole trap and laser cooling in 1978 by Dehmelt and collaborators[47].

For ${ }^{136} \mathrm{Ba}^{+}$ions there is a strong allowed transition at $\sim 493 \mathrm{~nm}$ between the ground state $6^{2} S_{1 / 2}$ and the first excited state $6^{2} P_{1 / 2}$ and a weaker transition between the 
$6^{2} S_{1 / 2}$ state and the metastable state $5^{4} D_{3 / 2}$ separated by $\sim 650 \mathrm{~nm}$. The first transition has a spontaneous lifetime of $7.74 \pm 0.4$ ns and when saturated radiates $\sim 6 \times 10^{7}$ photons/s. The branching ratio of the decay into the $\mathrm{D}$ and S states is $26.5 \pm 2 \%$ and $73.5 \pm 2 \%$ respectively [51].

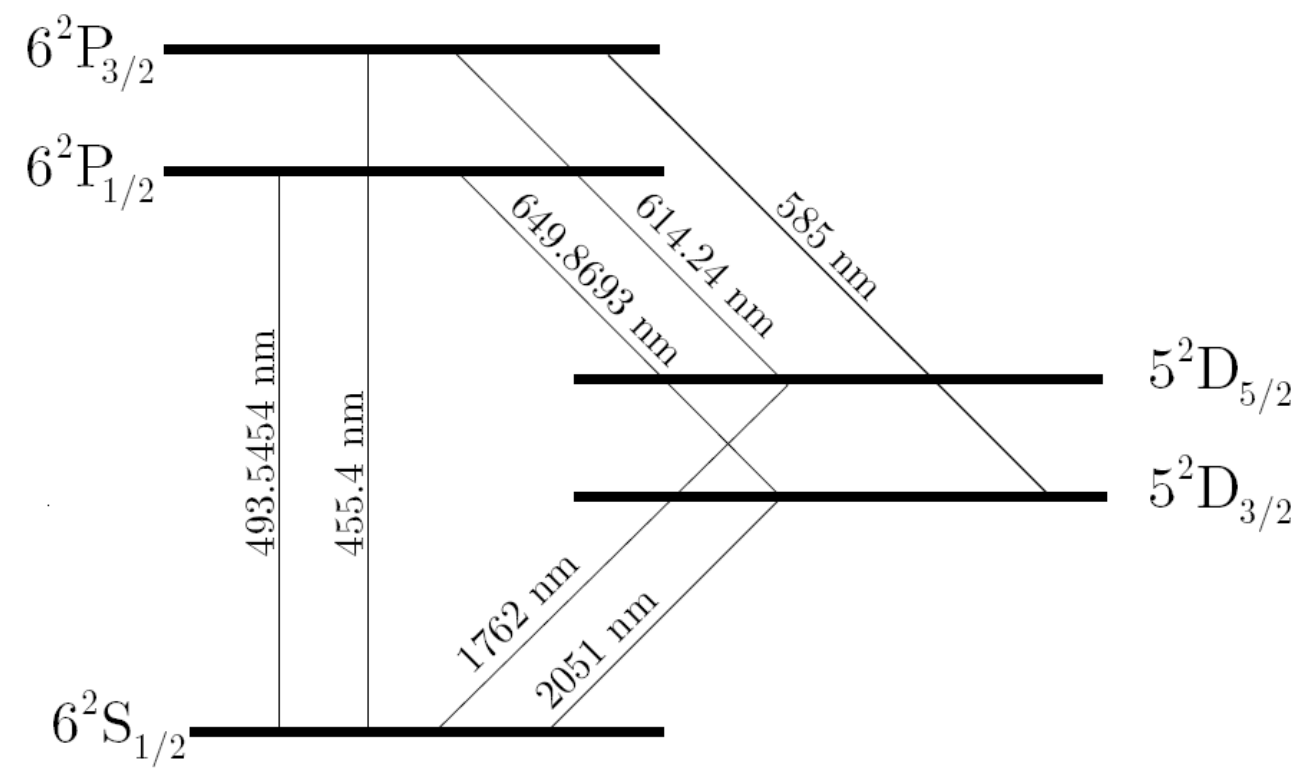

Figure 3.4: Atomic level scheme for $\mathrm{Ba}^{+}$ions. Wavelength values for transitions taken from [48]. Adapted from [49].

Because the atomic level structure is a unique print of the ion to study, laser induced fluorescence (LIF) is a very reliable technique to perform the identification of ${ }^{136} \mathrm{Ba}^{+}$ions. The process consists in exciting the ground state to the first excited state $\left(6^{2} S_{1 / 2}-6^{2} P_{1 / 2}\right)$ of the barium ion by using a laser tuned at $493 \mathrm{~nm}$. When it decays back to the ground state after $7.74 \pm 0.4 \mathrm{~ns}$, it would produce a fluorescence photon that can be detected. When the excited state decays into the metastable state $\left(6^{2} S_{1 / 2}-5^{4} D_{3 / 2}\right)$, as it is expected $\sim 26.5 \%$ of the time, it is necessary to re excite the ion from the $5^{4} D_{3 / 2}$ state to the $6^{2} P_{1 / 2}$ state by using a laser tuned at 650 $\mathrm{nm}$. To maximize the number of photons produced by this process, both lasers must run continuously. 


\subsubsection{General Concept}

Despite the fact that LIF is a relatively easy technique to develop, there are a series of complications when applying the concept for barium tagging for a gaseous Xe detector. The daughter product of the $\beta \beta$ decay of xenon is a doubly charged $\mathrm{Ba}$ ion, $\mathrm{Ba}^{++}$. The ground state to first excited state excitation wavelengths for $\mathrm{Ba}^{++}$are in the vacuum ultraviolet, which makes its detection not feasible, as suitable lasers for excitation do not exist. In order to identify such ion via laser induced fluorescence, it is necessary to neutralize it to either a singly charged state, $B a^{+}$, or a neutral state, $B a$. The change in ionization state does not occur by collisions with the Xe or by neutralization by the trail of electrons produced in the $\beta \beta$ decay. The ionization potential of $\mathrm{Xe}(12.127 \mathrm{eV})$ is greater than such of $B a^{+}(10.001 \mathrm{eV})$ or $B a(5.2 \mathrm{eV})$ and the fraction of neutralized ions by the electron trail is negligible. Therefore, the conversion between $\mathrm{Ba}^{++}$to either $\mathrm{Ba}^{+}$or $\mathrm{Ba}$ must be achieved by charge exchange collisions with a low ionization potential gas.

If the barium ion is in high pressure gas, the optical transitions will be pressure broadened and to achieve a fluorescence rate at the level used in traps it is necessary to increase the laser power by a factor of 1000 , causing backgrounds due to the scattered photons and making the process much more complicated. It is desired then, to extract and trap the neutralized barium ion into a vacuum chamber where it can be identified using known laser induced fluorescence techniques.

The general concept consists of a TPC operating at 10 bar with enriched xenon in the isotope ${ }^{136} \mathrm{Xe}$. Scintillation and ionization signals would be recorded through an electroluminescence process to determine the energy and position of events occurring in the detector in a similar way to the described one in the previous section for the gaseous Xe prototype. When an event occurs in the energy region of interest (around the $\mathrm{Q}$ value at $2458 \mathrm{keV}$ ), the electric field in the TPC would be modified to drift the ions towards an exit port where they would be extracted into a vacuum region. The ion would be pushed out from the detector by the xenon gas flow and would be separated from the gas using either an ion guide system (as used in mass spectrometry) or an RF funnel as proposed by Varentsov in 2004 [50]. Once the barium ion is separated from the xenon gas it would be analyzed and filtered with a 
quadrupole mass spectrometer, $\mathrm{Ba}^{++}$would be converted to $\mathrm{Ba}^{+}$and then trapped in a linear RFQ ion trap for further identification using laser induced fluorescence.

\subsubsection{Current Status}

Several efforts have been made among the EXO collaboration to prove the barium tagging concept in gaseous xenon, although a full working system has not been accomplished yet. In 2007, individual Ba ions were trapped and observed by resonance fluorescence with a high signal-to-noise ratio in a gas-filled linear ion trap designed by the EXO collaboration $[52,53]$. The barium source used for this purpose was chemically produced by heating a barium dispenser loaded with $B a A l_{4}-N i$, and depositing $\mathrm{Ba}$ on a Ta foil [53].

In 2011, $\mathrm{Ba}^{++}$conversion to $\mathrm{Ba}^{+}$was achieved using a triple quadrupole mass spectrometer, where doubly charged barium ions in the collision cell were contained using a RF sextupole and neutralized to singly charged ions by the interaction with triethylamine (TEA), a low ionization potential gas. Although during the process there was no evidence for the formation of any other species or molecules, it was found however that such gas was highly contaminant and it would remain in the system for long periods of time. The barium source used for this purpose was barium acetate dissolved in a mix of water and methanol and injected in the system using an electrospray ion source operated at atmospheric pressure [54].

An ion beam extraction system is currently being developed by collaborators at Stanford based on the RF funnel proposal by V.L. Varentsov [50]. The RF ion funnel device consists of a stack of 301 electrodes placed besides a converging-diverging nozzle at the exit plane, where Ba ions from a Gd driven ion source are injected into the funnel via a supersonic Xe gas jet. Pumping the funnel region evacuates the Xe gas from the beam line and at the same time the applied RF field cones the Ba ions inside the funnel for transport into a vacuum region. Details of the setup are described in [55].

A mass spectrometer with a nanoelectrospray source is currently operating at Carleton, producing $\mathrm{Ba}^{++}$and $\mathrm{Ba}^{+}$ions at pressures between 1 and 15 psig and being 
extracted into vacuum using insulated ceramic nozzles with conducting surfaces offering higher efficiencies and lower gas flows. The system, which concept and operation is described in this work, consists of a nanoelectrospray ion source, a sextupole ion guide (SPIG), a quadrupole filter (commercial unit from EXTREL) and a channeltron ion detector. This setup once combined with the ion extraction system, the collision cell for charge conversion of the barium ion, the linear trap and corresponding tuned lasers will constitute a full working barium tagging system for a gaseous Xe detector for the EXO experiment. 


\section{Chapter 4}

\section{Mass Spectrometry Theory}

Neutrinoless double beta decay of ${ }^{136} \mathrm{Xe}$ offers the possibility of its detection in a background free environment by performing the identification of the barium daughter of the reaction using laser induced fluorescence. Since a $\mathrm{Ba}$ ion is $\sim 27,000$ times heavier than an electron its drift velocity in xenon is considerably slower. This fact would allow sufficient time to reconstruct the electron tracks of the neutrinoless beta decay event and locate the $\mathrm{Ba}^{++}$in the $\mathrm{x}-\mathrm{y}$ plane of the detector, while the $\mathrm{z}$ direction of the ion would be determined by the drift time. The barium ion would then be extracted from the xenon chamber to a lower pressure region and then to vacuum where a set of multipole ion guides would transport the ion away from the jet allowing for mass identification, selection and ion counting for further trapping and laser tagging.

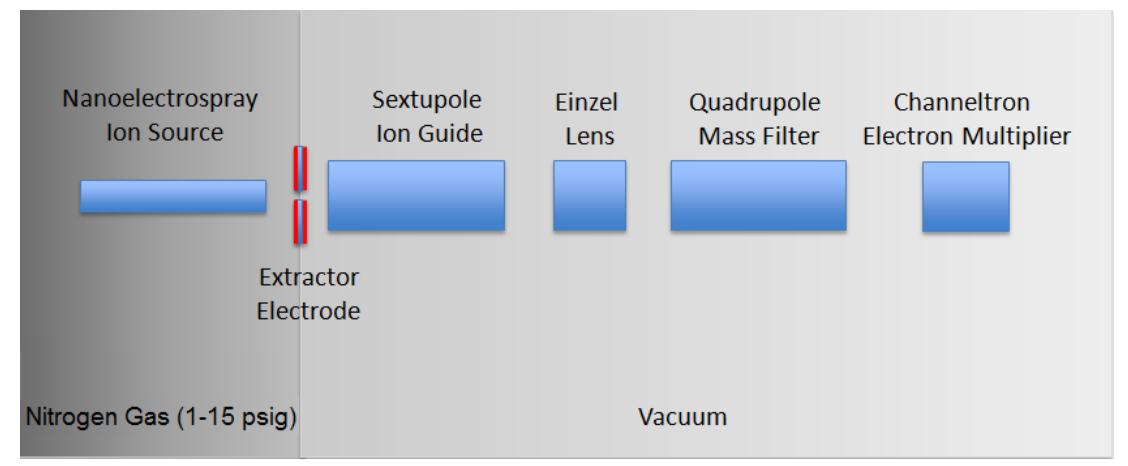

Figure 4.1: Diagram of the barium ion extraction and counting system.

The configuration of the barium ion extraction and counting system developed for 
this work is showed in figure 4.1. Barium ions are extracted from a barium acetate solution using a nanoelectrospray ion source which resides in a chamber full of nitrogen at pressures between 1 and 15 psig. Ions are drifted through an insulated orifice in a gold coated ceramic nozzle which plays the role of an extractor electrode and offers high transmission efficiencies and lower gas flows. An intermediate pressure region holds a sextupole ion guide (SPIG) where ions are confined and the remaining uncharged gas is pumped out of the system; ions are focused by an Einzel lens into a quadrupole mass filter where they are mass identified, selected and counted with a channeltron electon multiplier detector. This concept constitutes a mass spectrometry system that approaches some of the main problems in the extraction of barium ions from xenon: this includes reduction of gas flow into the system, ion mass filtering since the ion and gas masses are the same, and rapid ion extraction into vacuum preventing losses due to ion heating.

This chapter will outline the theory behind the operation of the elements that constitute the counting system for the barium extraction test facility developed for this work. Section 4.1 will present the principles and characteristics of nanoelectrospray ionization, the Taylor cone theory for spray formation and the type of electrodes used for ion extraction. Section 4.2 will cover the theory for the operation of sextupole and quadrupole ion guides, the purpose of the Einzel lens and will describe the operation of the Channeltron Electron Multiplier.

\subsection{Electrospray Ionization}

Electrospray ionization is a process that allows the extraction of ions from a solution into a gas phase free of solvent by applying an electric field to the tip of an emitter containing a conductive sample. This technique has applications in various fields such as engineering (for spray painting), mass spectrometry (for mass spectral analysis), and biochemistry (for creation of tissue cells by electrospraying biomolecules) among others.

Electrodispersion of fluids in air was first investigated by Zeleny [65]. He observed experimentally that a cone-jet was formed at the exit of a capillary needle when 
an electric field was applied to a dilute solution of hydrochloric acid contained in it. Many years later, Taylor [66] showed theoretically that conical fluid interfaces with a semi-vertical angle of $49.3^{\circ}$ were static solutions of the electrohydrodynamic equations, providing an explanation of the conical shape of the jet obtained when a liquid was subject to an electric field. This theory assumes that the surface of the cone is an equipotential surface and is based on the static equilibrium between the surface tension force and the electrostatic force on the surface of the fluid submitted to a high electric field. Properties of Taylor cone jets in air and vacuum, including stability, diameter of the jet, angle of spray, charge and distribution of the droplets, flow rate, current, applied voltage and flow rate are an ongoing topic of study.

A diagram of the electrospray ionization mechanism is shown in fig 4.2. An emitter (eg. syringe, needle) is filled with a conductive solution. Typical solvents used are mixtures of water with volatile organic compounds such as methanol or acetonitrile, and acetic acid is usually added to the solution to increase the conductivity of the sample and decrease the initial droplet size facilitating the ionization process. A large $(>1000 \mathrm{~V})$ applied potential difference applied between the emitter and an extractor electrode induces the formation of a fluid cone shaped jet at the tip of the emitter. The solvent starts to evaporate from the charged droplets until they reach their Rayleigh limit (the maximum amount of charge a liquid droplet can carry). The droplets then undergo Coulomb fission, where a fragmentation sequence occurs due to the fact that electrostatic repulsion of alike charges become stronger than the surface tension holding the droplets together. Inert gases such as nitrogen and carbon dioxide are often introduced to help decrease the surface tension between the droplets. The produced charged particles range in the orders of $\mu m$ to $\mathrm{nm}$ depending on the fluid and emitter properties. The charge of the particles depends on the potential difference applied. 


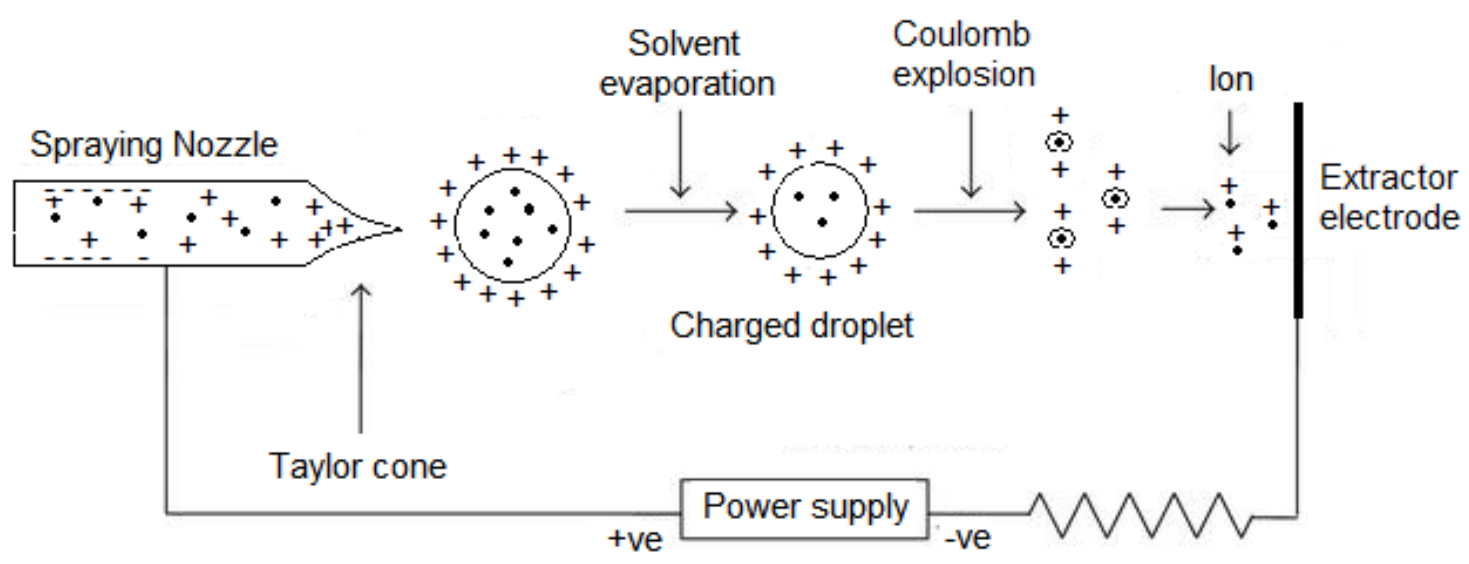

Figure 4.2: Diagram of the electrospray ionization mechanism. Adapted from [64].

Although electrospray ionization is a very powerful method and is widely used in various analytic investigations, there are some cases where it has been found that this technique has limited ionization efficiency (e.g hydrophilic compounds) with repercussions in analyte sensitivity and detection. The concept has been improved to decrease the amounts of sample used for analysis and increase sensitivity by lowering the flow rates to the nano scale. Such technique is known as nanoelectrospray ionization reaching sample flow rates of $<100 \mathrm{~nL} /$ min by using emitters with micron sized tip diameters.

\subsubsection{Nanoelectrospray Ionization}

Nanoelectrospray ionization is recognized as the most efficient technique, in terms of sample consumption, of producing ions from a liquid sample for direct analysis using mass spectrometry. Essentially, nanoelectrospray is a form of electrospray that is explained under the mechanism by which droplets follow multiple fragmentations by Coulomb fission when a DC electric potential is applied to the solution. The emitters used for nanoelectrospray are typically metal coated glass or quartz capillaries with tip diameters in the order of the $\mu m$. Such capillaries (needles) have sample flow rates between 1-100 $\mathrm{nL} / \mathrm{min}$ depending on the diameter of the tip, the voltage applied and the pressure at which they are operated. 
The advantages of nanoelectrospray ionization with respect to electrospray ionization are based on the fact that having a lower solution flow rate, reduced sizes of the initial charged droplets are obtained [72, 73]. Having smaller droplets at the initial stage require fewer fission fragmentations and less solvent evaporation is required to extract the ions into the gas phase. Therefore, a larger amount of ions are available for mass spectral analysis and the concentration of contaminants is reduced.

Such advantages have been observed in various experimental scenarios. For instance, purely aqueous solutions have been sprayed without the need of adding organic dopants [69], carbohydrates have been detected with high sensitivities without the need of applying chemical derivatization and ionization has been achieved without the use of solvent pumps or inlet valves [70]. Due to the high flow rates in the range of $1 \mu l$ to $1 \mathrm{ml}$ per minute, electrospray ionization is very sensitive to the presence of contaminants and salts in the analyte solution. The rapid evaporation causes formation of deposits at the exit of the emitter causing substantial suppression of the mass spectrum signals [68]. Nanoelectrospray has been shown to reduce such interference effect from salts due to its low flow rates, providing better sensitivities and the possibility of longer measurements for the analysis of samples [70].

Despite the number of improvements achieved using nanoelectrospray ionization, this technique also has some disadvantages in terms of signal intensity reproducibility. For a given solution, the obtained mass signals present variations upon the use of different nano-ESI needles. This problem is caused by the different shapes and diameters of the tip emitters and by the handling procedure of the nanoelectrospray needles. The emitter aperture size determines the liquid flow rate and therefore the size of the droplets in the fragmentation process. This fact restricts the previously mentioned advantages to very specific emitter parameters and conditions that can be complicated to reproduce.

\subsubsection{Extractor Electrode}

For the extractor electrode, skimmers are commonly used in mass spectrometry systems. They are metal discs with a small aperture hole of $\sim 1 \mathrm{~mm}$ in the center. Their 
main purpose is to sample the center of the ion beam coming from the electrospray emitter and extract it towards a vacuum region for further identification using a quadrupole mass analyzer.

The main disadvantage of such extractors is the size of their aperture holes. The flow rate $Q$ of a gas passing through an orifice plate is given in the literature by [71]

$$
Q \propto A \sqrt{2 \frac{Z R T}{M}\left(\frac{k}{k-1}\right)\left[\left(\frac{P_{2}}{P_{1}}\right)^{2 / k}-\left(\frac{P_{2}}{P_{1}}\right)^{(k+1) / k}\right]}
$$

where $A$ is the cross sectional area of the orifice hole in $m^{2}, Z$ is the gas compressibility factor at a given temperature $\mathrm{T}$ and pressure $P_{1}, M$ is the gas molar mass in $\mathrm{kg} / \mathrm{mol}, \mathrm{R}$ is the universal gas law constant $R=8.3145 \mathrm{~J} / \mathrm{molK}, k$ is the specific heat ratio between the upstream and downstream side of the orifice which are at pressures $P_{1}$ and $P_{2}$ in $\mathrm{Pa}$ respectively.

To reduce the gas flow rate passing through the extractor into the vacuum system housing the quadrupole analyzer, an alternative approach consists of coupling the nanoelectrospray source to smaller aperture sizes of the order of the $\mu m$. This configuration requires higher electric fields to guide the ions efficiently. For the purpose of this work, an insulated ceramic nozzle with conducting surfaces was fabricated to test ion extraction efficiencies and achieve lower gas flows into the system.

\subsubsection{Taylor Cone Theory}

In 1964, Taylor [66] developed a theory to explain the characteristic conical shape of the formed jet in the electrospray ionization mechanism. This section will describe the principal aspects of such theory for a steady cone jet mode as described by Taylor in a static case in which no fluid dispersion occurs. In this case, the electric field would be orthogonal to the surface, which is assumed to be conductive, and the main forces acting on it are the surface tension and the electrostatic force. 


\subsubsection{The Taylor Cone}

In an electrospray system, when a potential difference is applied between the emitter and the extractor electrode the electrostatic force tends to pull the liquid out of the emitter attracting it towards the extractor electrode plate. The surface tension on the other hand tends to retract the fluid on the opposite direction minimizing its surface area. For the static case in which no liquid is sprayed, Figure 4.3 shows a schematic of the liquid Taylor cone.

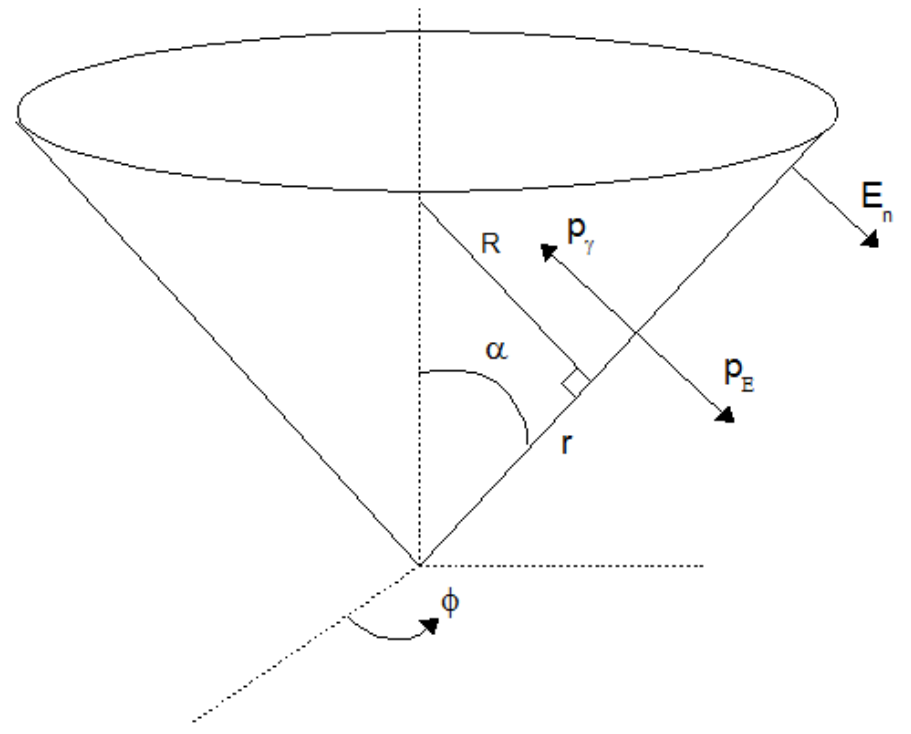

Figure 4.3: Diagram of the liquid Taylor Cone. $\alpha$ is the cone angle, $p_{\gamma}$ and $p_{E}$ are the surface tension and electrostatic pressures respectively, $E_{n}$ is the electric field on the cone surface, and $R$ is the radius of curvature. Adapted from [74]

If the fluid surface is treated as a perfect conductor, the electrostatic pressure $p_{E}$ on it is given by

$$
p_{E}=\frac{\varepsilon_{0}}{2} E_{n}^{2}
$$

where $\varepsilon_{0}$ is the permittivity of free space and $E_{n}$ is the electric field normal to the fluid surface. 
The surface tension pressure $p_{\gamma}$ of the liquid would be given by

$$
p_{\gamma}=\gamma\left(\frac{1}{R}\right)=\gamma\left(\frac{1}{r \tan \alpha}\right)
$$

where $\gamma$ is the surface tension of the fluid, $R$ is the radius of curvature $\left(\kappa=\frac{1}{R}\right.$ is the curvature of the surface) which can be expressed from figure 4.3 in terms of the cone angle $\alpha$, as $R=r \tan \alpha$.

Assuming there are no other forces acting on the surface, the pressures due to the electrostatic force and due to the surface tension must be equal in magnitude at all points of the cone surface, that is $p_{E}=p_{\gamma}$ and from equations (4.2) and (4.3) we obtain the normal electric field

$$
E_{n}=\sqrt{\frac{2 \gamma}{\varepsilon_{0}}\left(\frac{1}{r \tan \alpha}\right)}
$$

from where the electric potential results

$$
\Phi(r, \alpha)=\phi_{0} r^{\frac{1}{2}} P_{1 / 2}(\cos \alpha)
$$

where $P_{n}$ is the Legendre Polynomial of degree $n$.

For the surface to be equipotential and independent of $r$, the solution must satisfy that $P_{1 / 2}(\cos \alpha)=0$ with $\phi=0$, that is, the liquid cone semi-angle $\alpha$ must be equal to $49.3^{\circ}$. This result was first resolved and tested experimentally using liquid metals in different emitter shapes by Taylor in 1964 [66]. The limitations of this model rely on the fact that a perfectly conducting liquid is assumed when in reality the solutions used in analytical chemistry electrospray are generally of low conductivity. However, Taylor's description provides an explanation of why a cone shape is formed and is still valid for most cases. In the dynamic case, there is a voltage drop over the liquid cone and the cone angle becomes smaller than $49.3^{\circ}[75]$. 


\subsubsection{Cone Threshold Voltage}

The Taylor cone is formed at the tip of the emitter when the applied electric field is high enough for the process to be stable. The threshold voltage $U_{T}$ is defined as the potential difference between the emitter and extractor electrode at which the cone jet electrospray is constant. Before such voltage is reached, the spray consists of intermittent cone shapes that collapse and prevent the formation of a steady cone jet.

Wilm and Mann [74] present a mathematical description of the dependence of the threshold voltage on the distance between the tip of the emitter and the extractor electrode assuming an elliptically shaped fluid surface to approximate the shape of the Taylor cone. Their results are presented below.

For an electric potential containing the Taylor cone potential and a perturbation term, the result for the electrostatic pressure on the edge of the elliptic fluid cone is given by

$$
p_{\gamma}-p_{E}=\frac{1}{r_{a}\left(2 \gamma-\frac{2.68 \varepsilon_{0} U_{a}^{2}}{r_{1}}\right)}
$$

where $p_{\gamma}$ and $p_{E}$ are the surface tension and electrostatic pressures respectively, $\gamma$ is the surface tension of the fluid, $\varepsilon_{0}$ is the permittivity of free space, $U_{a}$ is the potential difference applied between the emitter and the extractor electrode, $r_{1}$ is the distance from the tip of the cone to the extractor electrode and $r_{a}$ is the radius of curvature of the circle formed at the tip of the cone.

When $U_{a}$ reaches the threshold, the liquid is pulled out of the emitter forming half an ellipsoid at the tip of the emitter. At this point, the surface tension and electrostatic pressures must be equal to each other in order to allow the change of shape to a pointed cone. The dependence of the threshold voltage on the distance $r_{1}$ is given by

$$
U_{T}=0.863\left(\frac{\gamma r_{1}}{\varepsilon_{0}}\right)^{\frac{1}{2}}
$$

For some electrospray fluids, due to the dependence of $U_{T}$ on the surface tension as $\gamma^{1 / 2}$, electrical breakdown occurs before the threshold voltage is reached. Such discharge can be prevented by operating the ion source in a gas at either high or low 
pressures to scavenge (remove) electrons from the tip of the emitter.

Equation (4.7) is limited to situations that satisfy the assumed geometry, where one has an elliptic fluid cone and a flat extractor electrode. Any change in the emitter tip radius affects the cone half angle, the fluid shape at the tip of the needle and thus the produced electrospray cone.

It has been observed experimentally that the threshold voltage for which the cone jet structure is constant and stable is obtained as the applied voltage to the emitter is increased. However, when the voltage is decreased from operating in steady cone jet mode to a point where the cone becomes unstable (also known as extinction voltage) it has been found that such extinction voltage is significantly lower then the threshold voltage. Such hysteresis is not well understood, although it is attributed to the variation with voltage of the cone surface shape $[76,77,78,79]$.

\subsubsection{Jet Formation}

When the flow of charges in the liquid is overtaken by the flow of charges towards the cone surface, a non equipotential surface is obtained and a jet is formed. The jet breaks up into charged droplets and the subsequent fragmentation into ions can occur in a number of different ways depending on the conditions at which the electrospray is operated.

Hartman et al [80] investigated the mechanism by which the cone jet breaks up using a high-resolution camera. In their paper, they present a model to predict droplet size and velocity of the jet as a function of the normal electric field and surface tension pressures and found that the jet break up process depends on the viscosity, surface charge and acceleration of the jet. Hartman et al suggested that as the ratio between the electric field stress and the surface tension stress $\left(R=\frac{p_{E}}{p_{\gamma}}\right)$ increases the jet break up mode changes, they identified three main modes of spray as shown in figure 4.4. 

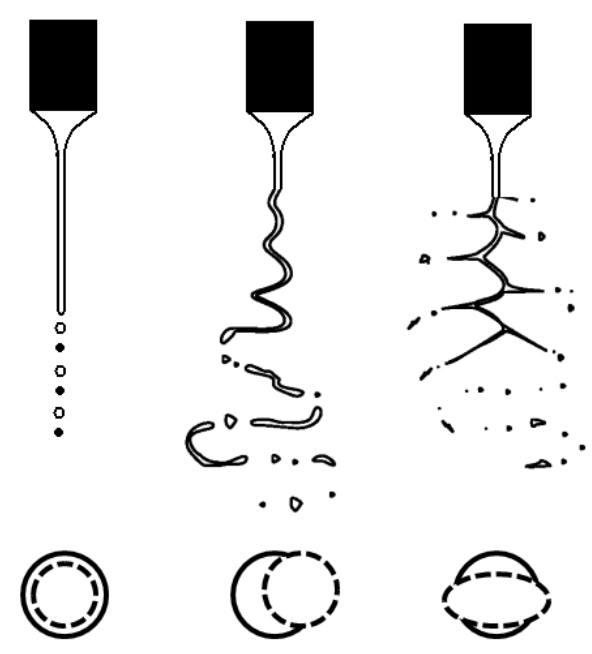

Figure 4.4: Types of jet break up as described by Hartman et al. From left to right, a symmetric, a lateral and a ramified jet break up. Adapted from [80].

From figure 4.4 three main modes of break up are observed previous to droplet formation, fragmentation and ion extraction. The first case results from the stable cone jet formation where the electric field stress to surface tension stress ratio $\mathrm{R}$ is unity. For low ratios, i.e for $p_{E}<p_{\gamma}$ instabilities in Rayleigh droplet formation result in a lateral break up; and for high ratio values, i.e $p_{\gamma}<p_{E}$ as the surface tension is not enough to counteract the electric field pressure, ramification of the jet occurs resulting in a non symmetric jet.

Any variations in the initial shape of the Taylor cone and on the downstream jet causes a non-uniform division of charged droplets of different shapes. Classically, the maximum charge a spherical droplet, of radius $r_{\text {droplet }}$, can have is known as the Rayleigh limit and is given by

$$
q_{\text {max }}=8 \pi\left(\varepsilon_{0} \gamma\right)^{1 / 2} r_{\text {droplet }}^{3 / 2}
$$

In the presence of volatile liquids and gas nebulisers repeated non-uniform droplet divisions occur until the point where ions are extracted from them. This constitutes the process by which electrospray ionization is performed. 


\subsubsection{Emission Radius}

Another interesting derivation presented by Wilm and Mann [74] involves the relation between the emission diameter of the droplets produced at the tip of the Taylor cone and the flow rate. The radius of the droplets at the tip of the Taylor cone is given by $[74]$

$$
r_{e}=\left(\frac{\rho}{3 \pi^{2} \gamma \tan \left(\frac{\pi}{2}-\alpha\right)\left[\left(\frac{U_{a}}{U_{T}}\right)^{2}-1\right]}\right)^{1 / 3}\left(\frac{d V}{d t}\right)^{2 / 3}
$$

where $\rho$ is the liquid density, $\gamma$ is the liquid surface tension, $\alpha$ is the cone angle, $U_{a}$ is the applied potential difference between the emitter and the extractor electrode, $U_{T}$ is the threshold voltage and $\frac{d V}{d t}$ is the flow rate.

This relation has important implications for the ion extraction efficiency because the size of the initial droplets determines the fragmentation efficiency along the jet. The flow rate has been found to depend on the applied voltage and current of the produced droplets and a number of studies are pursued towards the understanding of such observation.

\subsection{Multipole Ion Guides}

Radio frequency $(\mathrm{RF})$ ion guides have been used throughout the years for ion transport along optical ion paths. They have become widely used in commercial instruments to analyze biological and geological samples using techniques such as atmospheric pressure electrospray ionization mass spectrometry, liquid chromatography (LC) and tandem mass spectrometry (MS/MS). RF guides have been adapted for use as ion traps in a number of applications such as: ion containment for charge state separation techniques, ion-photon, ion-molecule and ion-ion interactions.

Multipole ion guides typically employ linear RF electrode geometries that follow a cylindrical symmetry. The time-varying electric potential of an ideal two-dimensional multipole configuration of order $n$ with infinite extension along the z-direction is 
given in cylindrical coordinates by [56]

$$
\Phi(r, \phi, t)=V_{0} \cos (n \phi)\left(\frac{r}{r_{0}}\right)^{n} \sin (\Omega t)
$$

where $V_{0}$ is the RF amplitude, $r_{o}$ is the inscribed radius of the electrodes and $\Omega$ is the angular frequency. This potential is created by an arrangement of $2 n$ cylindrical electrodes of radius $r_{e}$ placed on a cylinder of inscribed radius $r_{0}$ for different multipole orders $n$ as depicted in figure 4.5.
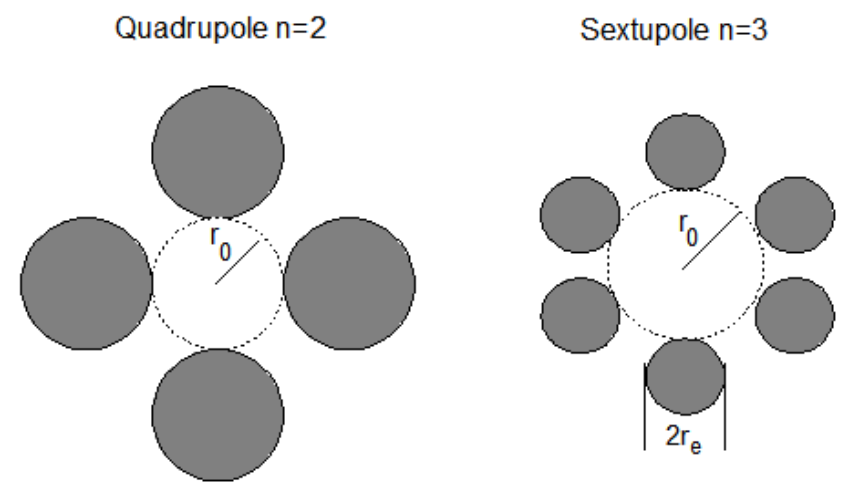

Octupole $n=4$

Figure 4.5: Cross section diagram of linear radio frequency multipole ion guides of order $\mathrm{n}=2,3$ and 4 .

To achieve a potential that optimally approximates the curvature of the ideal multipole potential to first and second orders, the diameter of the rods has to fulfill the relations $r_{e}=(1.146) r_{0}[61], r_{e}=(0.5375) r_{0}[61], r_{e}=(0.355) r_{0}$ [62], respectively for quadrupole, sextupole and octupole ion guides.

The principle of ion confinement due to RF fields in a multipole ion guide is described by the so called pseudopotential well model. For the ideal two-dimensional multipole field of equation (4.10) ion trajectories are determined approximately by an effective mechanical potential, $\varphi(r)$, given by [56]

$$
\varphi(r)=\frac{n^{2} e V_{0}^{2}}{4 m \Omega^{2} r_{0}^{2}}\left(\frac{r}{r_{0}}\right)^{2 n-2}
$$

where $2 n$ is the number of poles, $e$ is the electron charge, $V_{0}$ is the RF voltage (zero 
to peak) applied to one pair of rods, $m$ is the ion mass, $\Omega=2 \pi f$ is the RF angular frequency, $f$ is the frequency in $\mathrm{Hz}$ of the RF signal and $r_{0}$ is the inscribed radius of the multipole. Figure 4.6 compares the well profiles for quadrupole, sextupole, and octupole ion guides of the same interior radius.

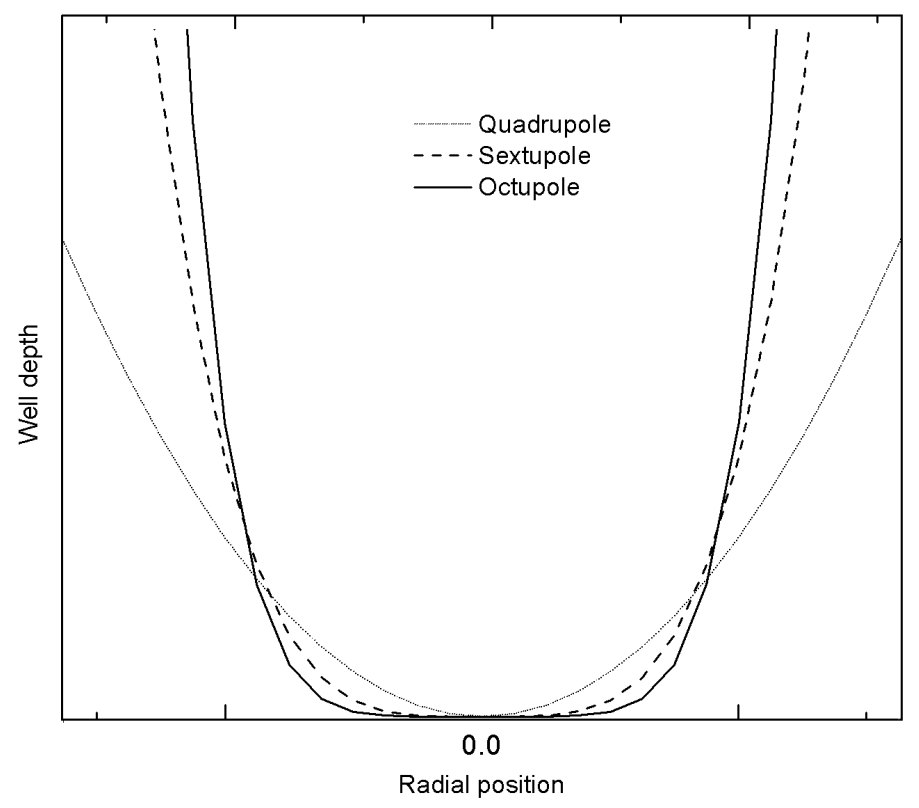

Figure 4.6: Pseudopotential well model for a quadrupole, sextupole and octupole with arbitrary $r_{0}, \Omega$ and $V_{0}$ values.

According to figure 4.6, the pseudopotential well model predicts that higher order multipoles provide stronger ion confinement (higher walls), preventing loss of ions with high radial energies and providing stable conditions for a wider mass range of ions at a given RF voltage. Due to the flatter profiles of the pseudopotential well for sextupoles and octupoles, it is expected that they produce wider ion beams at the exit. However, the narrow beam that is produced at a quadrupole exit is advantageous for ion transmission through small apertures and for ion confinement in small radial regions, which for example can provide better interactions with laser beams. 

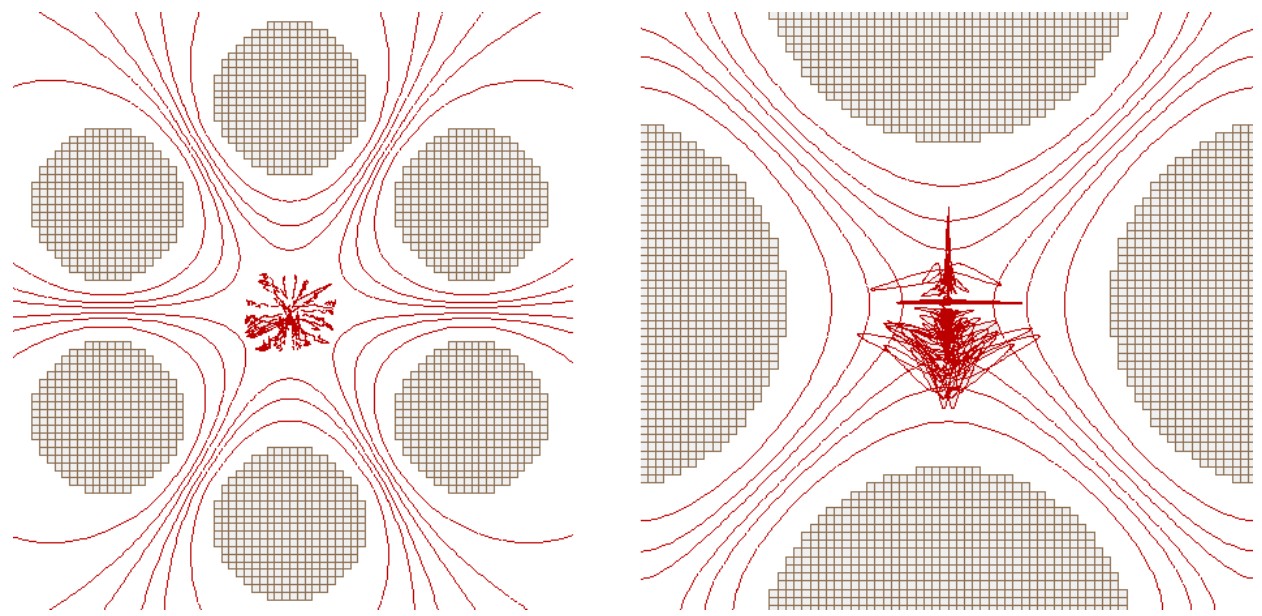

Figure 4.7: Simulated stable ion trajectories for ions traveling within a sextupole and quadrupole field.

A simulated example of stable ion trajectories for a sextupole and quadrupole are shown in figure 4.7. The applied RF voltage and frequency determine the electric field that cause ion confinement within the axis of the multipole.

\subsubsection{Ion Motion in a 2D Multipole Field}

The motion of an ion in a two dimensional multipole field is determined by Newton's Law,

$$
\vec{F}=m \frac{d \vec{v}}{d t}
$$

where $m$ is the ion mass, $\vec{v}$ is the ion velocity and $\vec{F}$ is the force that a charged particle feels within the multipole. $\vec{F}$ is defined as the magnitude of the electric field multiplied by the charge of the particle,

$$
\vec{F}=-e \nabla \Phi(x, y, t)
$$

where $e$ is the magnitude of the electronic charge and $\Phi$ is the multipole potential.

In the absence of collisions, ions move freely along the central axis of the multi- 
pole and the motion in the $x$ and $y$ directions is determined by

$$
\begin{aligned}
& m \frac{d^{2} x}{d t^{2}}=-e \frac{\partial \Phi(x, y, t)}{\partial x} \\
& m \frac{d^{2} y}{d t^{2}}=-e \frac{\partial \Phi(x, y, t)}{\partial x}
\end{aligned}
$$

\subsubsection{Sextupole Ion Guide (SPIG)}

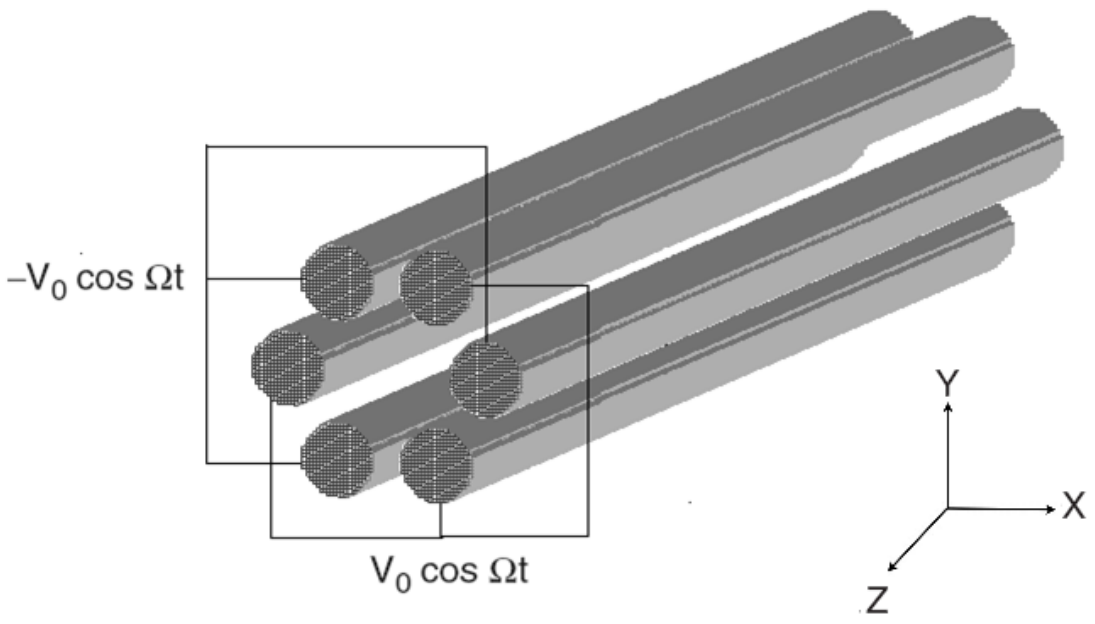

Figure 4.8: Diagram of a sextupole. Cylindrical rods in an hexagonal array.

A sextupole is an hexagonal array of rods operated in an RF only mode commonly used for either efficient ion transport between two stages of an optical ion path, or for production of secondary ions by induced dissociation due to collisions with a buffer gas. For the first case such device is commonly known as a Sextupole Ion Guide (SPIG), and for the latter case it is known as an Hexapole Collision Cell.

Because the potential well created by the RF potential associated with a SPIG does not have such a sharp minimum as that of a quadrupole and because the potential walls are higher and steeper, sextupoles are ideal devices to transport high current beams and to couple high pressure ion sources to quadrupole mass analyzers. In con- 
trast to the quadrupole, for a sextupole the $\mathrm{x}$ and $\mathrm{y}$ directions of motion are strongly coupled to each other and the frequencies of ion oscillation and the stability of the trajectories depend mainly on the initial conditions of the ions. Because there is no applied DC voltage on the sextupole electrodes, SPIGs are not capable of performing ion mass discrimination.

\subsubsection{Einzel Lens}

An Einzel lens consists of an array of cylindrical electrodes arranged in series used for ion beam focusing purposes. For the case of the setup developed for this work, the Einzel lens is used to couple the sextupole ion guide to the quadrupole mass analyzer by focusing the ions coming into it. This is achieved by manipulating the electric field along the ion path. A great advantage of this ion optical tool is the fact that it focuses the ion beam without changing the energy of the ions, and thus the ion velocity. A diagram of the Einzel lens configuration for a positive ion beam is shown in figure (4.9)

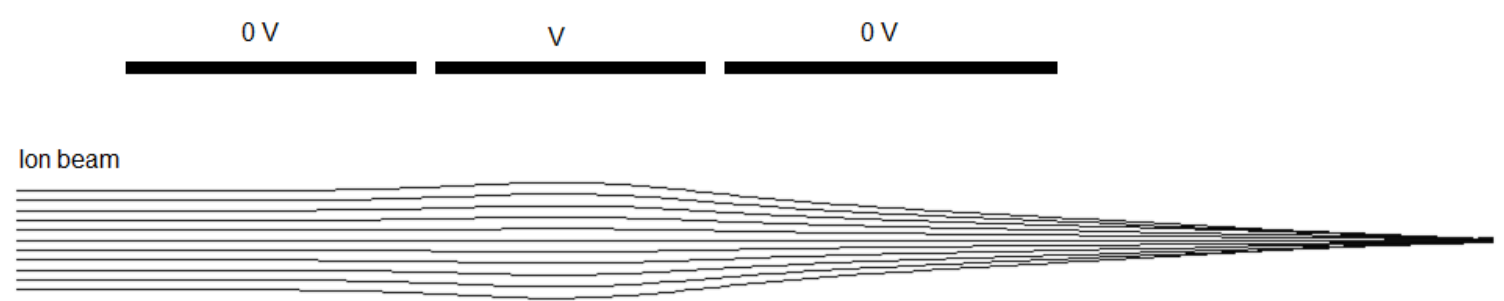

Figure 4.9: Diagram of Einzel lens and corresponding ion path. The voltage polarity of the middle electrode depends on the polarity of the ion beam.

\subsubsection{Quadrupole Mass Filter}

A quadrupole mass filter is an assembly of four parallel metal rods as shown in figure 4.10. An equal but opposite DC voltage $(U)$ superimposed with a radio frequency $\left(V_{0}\right) \mathrm{AC}$ voltage is applied to the diagonally placed pair of rods so that electrode pairs are out of phase with each other. The resulting electrical field causes ions 
entering the quadrupole to travel forward in the $\mathrm{z}$ direction with oscillatory motion in the $\mathrm{x}-\mathrm{y}$ plane. The amplitude of oscillation constitutes a unique property of the mass to charge ratio of the traveling ions and can be controlled by changing the DC and RF voltages simultaneously. This way, only those ions with stable trajectories at the operating DC and RF will travel the length of the filter and reach the detector.

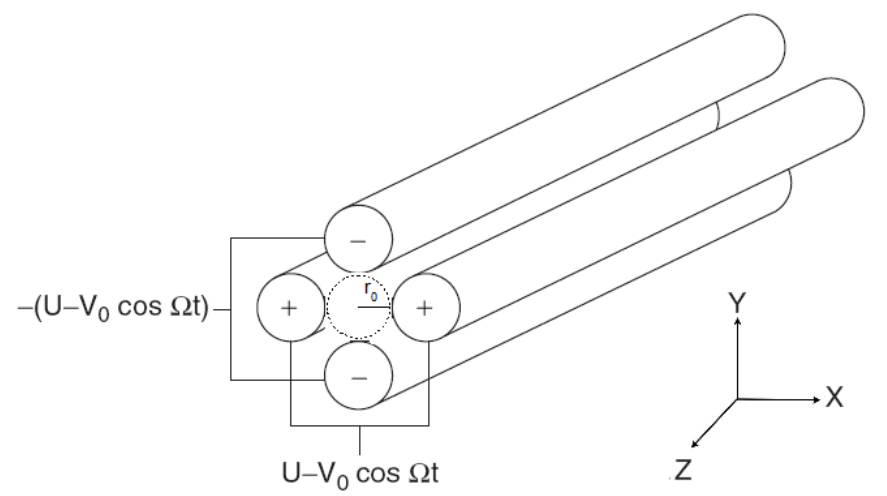

Figure 4.10: Diagram of a quadrupole mass filter. Ions would oscillate in the $\mathrm{x}-\mathrm{y}$ plane and travel in the $\mathrm{z}$ direction.

The field in the $\mathrm{x}-\mathrm{z}$ plane of a quadrupole acts as a high mass pass filter. Lighter ions oscillate in phase with the RF potential and the amplitude of oscillation increases until the ion is lost either by contact with the rods or by ejection from the array. Heavier ions remain near the center of the quadrupole and are emitted through the exit aperture towards the detection device. The $\mathrm{y}-\mathrm{z}$ plane on the other hand acts as a low mass pass filter. Both heavy and light ions are attracted toward the negative DC potential of the rods, but light ions are refocused toward the center by the RF potential where they maintain stable trajectories.

The 2D quadrupole electric potential $\Phi$ is given by [58]

$$
\Phi(x, y, t)=\frac{\left(x^{2}-y^{2}\right)}{r_{0}^{2}} \Psi(t)
$$

where $r_{0}$ is the radius of the inscribed circle tangential to the inner surface of the rods, and $\Psi(t)$ is the potential applied to the electrodes. The minus sign between the $\mathrm{x}$ and the $\mathrm{y}$ directions is due to the opposite polarity of the horizontal and vertical 
rods.

If a potential $\Psi(t)= \pm\left(U-V_{0} \cos (\Omega t)\right)$ is applied to alternate electrodes of a quadrupole, where $U$ is the $\mathrm{DC}$ voltage, $V_{0}$ is the RF voltage and $\Omega=2 \pi f$ is the angular frequency with $f$ the frequency in $\mathrm{Hz}$, the $2 \mathrm{D}$ quadrupole potential is given by

$$
\Phi(x, y, t)=\frac{\left(x^{2}-y^{2}\right)}{r_{0}^{2}}\left(U-V_{0} \cos (\Omega t)\right)
$$

and equations (4.14) and (4.15) give the equation of motion in $\mathrm{x}$ and $\mathrm{y}$ directions respectively,

$$
\begin{aligned}
& m \frac{d^{2} x}{d t^{2}}=\frac{-2 e x}{r_{0}^{2}}\left(U-V_{0} \cos (\Omega t)\right) \\
& m \frac{d^{2} y}{d t^{2}}=\frac{+2 e y}{r_{0}^{2}}\left(U-V_{0} \cos (\Omega t)\right)
\end{aligned}
$$

Writing the equations of motion in terms of the parameters [59],

$$
a_{x}=-a_{y}=\frac{8 e U}{m \Omega^{2} r_{0}^{2}}
$$

and

$$
q_{x}=-q_{y}=\frac{4 e V_{0}}{m \Omega^{2} r_{0}^{2}}
$$

and defining the variable $\xi=\frac{\Omega t}{2}$, we obtain the so called Mathieu equations

$$
\begin{aligned}
& \frac{d^{2} x}{d \xi^{2}}+\left(a_{x}-2 q_{x} \cos 2 \xi\right) x=0 \\
& \frac{d^{2} y}{d \xi^{2}}+\left(a_{y}-2 q_{y} \cos 2 \xi\right) y=0
\end{aligned}
$$

The solutions to the Mathieu equations can be interpreted in terms of ion trajectory stability in the $\mathrm{x}$ and $\mathrm{y}$ directions, of ion confinement within the quadrupole field and of characteristic fundamental frequencies of ion motion in the $\mathrm{x}$ and $\mathrm{y}$ directions. Stable, bound solutions for the Mathieu equation in the $\mathrm{x}$ direction are obtained when the $a, q$ parameters lie in the region bounded by the characteristic 
curves $a_{0}$ and $b_{1}$ given by [60]

$$
a_{0}=-\frac{q^{2}}{2}+\frac{7 q^{4}}{128}-\frac{29 q^{6}}{2304}+\ldots
$$

and

$$
b_{1}=1-q-\frac{q^{2}}{8}+\frac{q^{3}}{64}+\frac{q^{4}}{1536}+\ldots
$$

The stability diagram for a linear quadrupole is shown in figure 4.11. Such graphical representation is useful to determine the voltage combinations that would lead to stable trajectories of ions of a specific mass to charge ratio taking into account the fact that both the size of the quadrupole rods and the frequency of the RF voltage are usually held constant in a particular quadrupole setup.

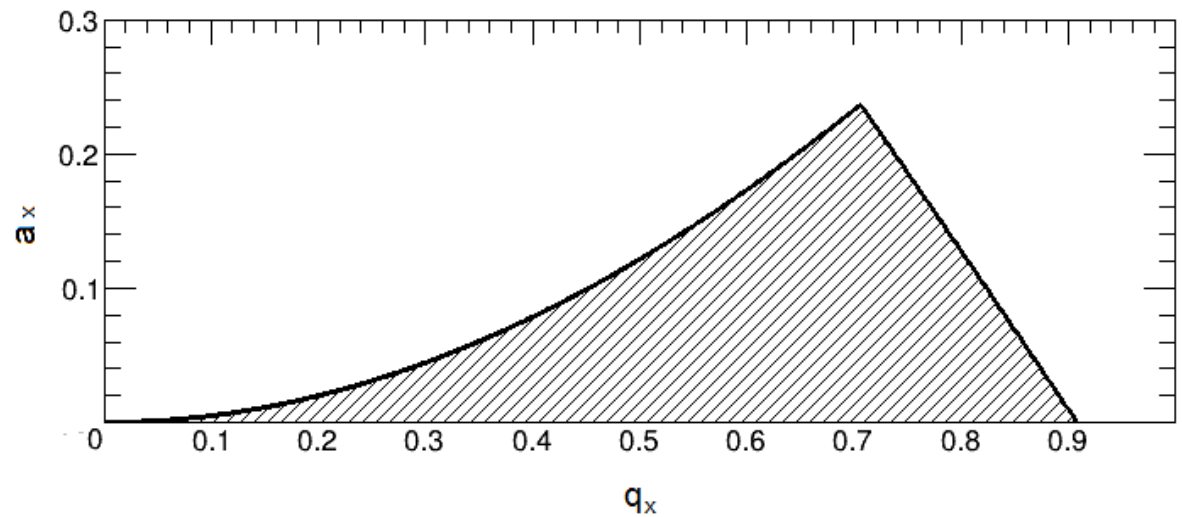

Figure 4.11: Stability region for a linear quadrupole with $r_{0}=8.23 \mathrm{~mm}$ at $f=1.2$ MHz and $m=136$ amu.

Quadrupole analyzers can be operated in two main modes: RF and DC mode or RF-only mode. When a quadrupole rod array is operated in a RF-only mode, that is $U=0$ in equation (4.20), all $a_{x}$ and $q_{x}$ coordinates lie on the $q_{x}$ axis in figure 4.11. In such case, all ions above a fixed mass to charge ratio are transmitted through the quadrupole device. When the device is operated in RF and DC mode, only ions within a small range of mass to charge ratio will maintain stable trajectories and will reach the detector allowing to perform mass selection of the incoming ions. The magnitudes of the DC and RF voltages must be varied by keeping a constant ratio between them so that the two filters (low mass and high mass) overlap allowing only 
a small range of mass to charge ratios to pass through the system and be detected.

\subsubsection{Channeltron Electron Multiplier}

A particle multiplier is an amplifying instrument that generates a pulse of charge at its output (anode) when an ion, electron or photon reaches its input dynode. The number of output charge pulses per second corresponds to the input number of ions per second, offering the possibility of performing ion counting with such device. The most common particle multipliers used in mass spectrometry include multi-stage CuBe types, continuous glass types and Conversion Dynode Electron Multipliers.

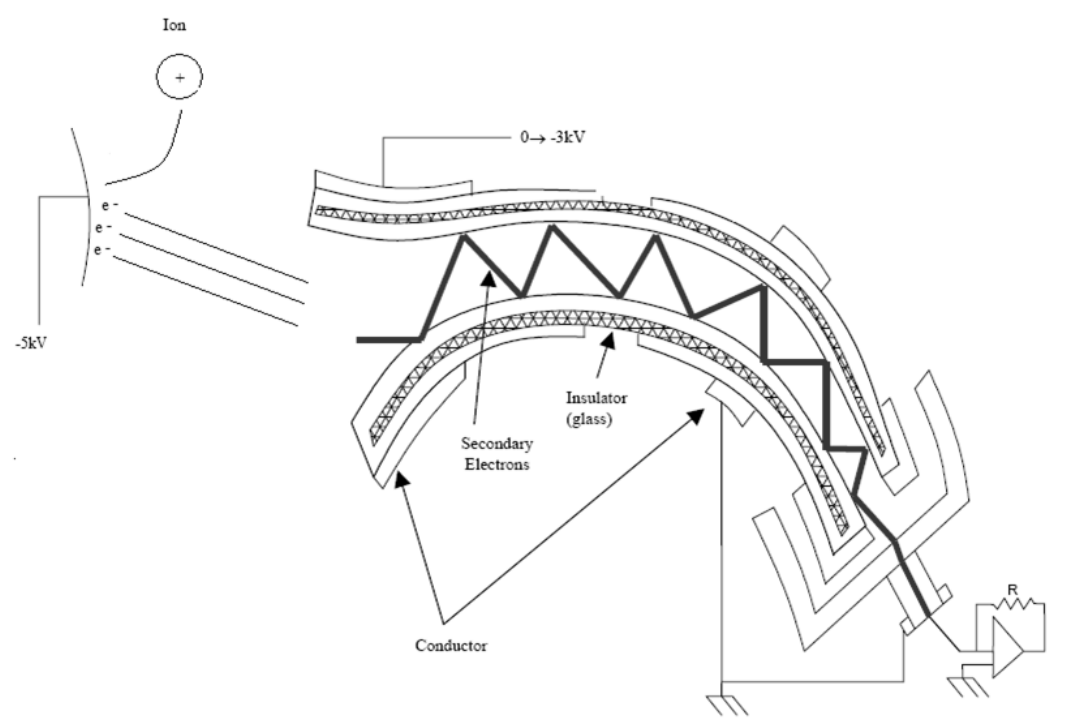

Figure 4.12: Diagram of the multiplier-dynode configuration for positive ion counting. Adapted from [81].

Figure (4.12) shows the internal configuration of a channel multiplier (CM) for positive ion detection. When a positive ion strikes the dynode (at $\sim 5 \mathrm{kV}$ ), it produces secondary electrons that are attracted towards the funnel shaped entrance of the multiplier (at $\sim 2 \mathrm{kV}$ ). The electric field along the path induces an avalanche effect when the electrons hit the surfaces of the device drifting them towards the anode end of the instrument where the signal is collected and further amplified. The polarity of the multiplier voltage determines the polarity of ions that will be detected; for negative ion detection a positive input bias is required and vice versa. 
The curved shape of the multiplier tubes is meant to prevent ionic feedbacks that could cause false output signals. When coupled to quadrupole mass analyzers, CMs are mounted off axis with respect to the analyzer exit aperture to avoid noise signals caused by discharge photons produced within the chamber as they would travel in a straight line through the detector housing. 


\section{Chapter 5}

\section{Ion Counting and Extraction System}

Although it is clear that the identification of the daughter product of the neutrinoless double beta decay of ${ }^{136} \mathrm{Xe}$ offers great advantages in terms on background reduction for the observation of such process, no proven facility in either gaseous or liquid xenon has been completely built yet. EXO-200 doesn't count with a barium identification system but it is expected that for the next phase of the experiment, either in liquid or gas xenon, such a scheme will be included. Since a measurement of the decay in a large scale detector will be highly dominated by backgrounds, the importance of having a barium tagging facility to reach neutrino mass sensitivities in the $50 \mathrm{meV}$ scale is recognized among the collaboration as a big priority and several efforts are being made to accomplish it within the time scale for the construction of the next phase detector.

In order to achieve a working barium tagging concept in a gaseous xenon TPC, it is necessary to perform a series of tests on each individual component of the system to understand its performance and limitations. As described in Chapter 2, the preferred technique to identify the barium ion product of the decay is laser induced fluorescence. The difficulties to apply such technique in situ, such as the charge conversion of the barium ion and the high vessel pressure, motivate the idea of extracting the ion into vacuum for charge conversion, mass filtering, trapping and further identification using LIF. 
The core of this work is the development of one of the components of the barium tagging facility for a gaseous xenon detector. It is a mass spectrometry system that consists of ion extraction, mass identification and counting devices to be coupled in the future to an ion trap that will hold the ions for LIF identification. The barium ion source incorporates a nanoelectrospray emitter operated in nitrogen at pressures between 1 and 15 psig (1 bar) with electrically insulated ceramic nozzles of different geometries for ion extraction into vacuum with minimum gas flows. Ions are confined in an ion optical path that includes a sextupole ion guide and an Einzel lens for transport towards a commercial quadrupole mass analyzer and channel electron multiplier for ion mass selection and counting.

Section 5.1 will describe the experimental setup for this system. The SIMION simulated expected performances under different pressure, voltage and geometry configurations of the ion source and extraction array are presented in Section 5.2. Finally, the experimental results of the operational system are presented in Section 5.3.

\subsection{Experimental Setup}

A diagram of the mass spectrometry system used for production, extraction and identification of barium ions is shown in figure 5.1. The system is mounted in two 8 " flange cross-shaped vacuum chambers, each one attached at the bottom port to a turbomolecular pump backed up by a scroll pump. Pressure inside the chamber is monitored by ion gauges mounted in the top ports. The ion source resides in a $2.75^{\prime \prime}$ ConFlat $(\mathrm{CF})$ chamber connected to a roughing pump with available feedtroughs for voltage and gas connections. It slides into the first chamber where the SPIG resides. The HV and RF feedtroughs for the skimmer plates and sextupole rods of the SPIG array are located in the back side port of the first chamber. The quadrupole analyzer is mounted on a $6^{\prime \prime}$ flange with the corresponding high voltage and RF feedtroughs. It is introduced in the right end of the second chamber and coupled to it using a flange reducer. The space between the two cross-shaped vacuum vessels contains the support for the SPIG array defining two pressure regions communicated by a 0.30 $\mathrm{cm}$ aperture at the end of the SPIG. The pumps used in the system are the same 
for both chambers, they are Edwards nEXT400D turbos backed by XDS-10 scroll pumps.

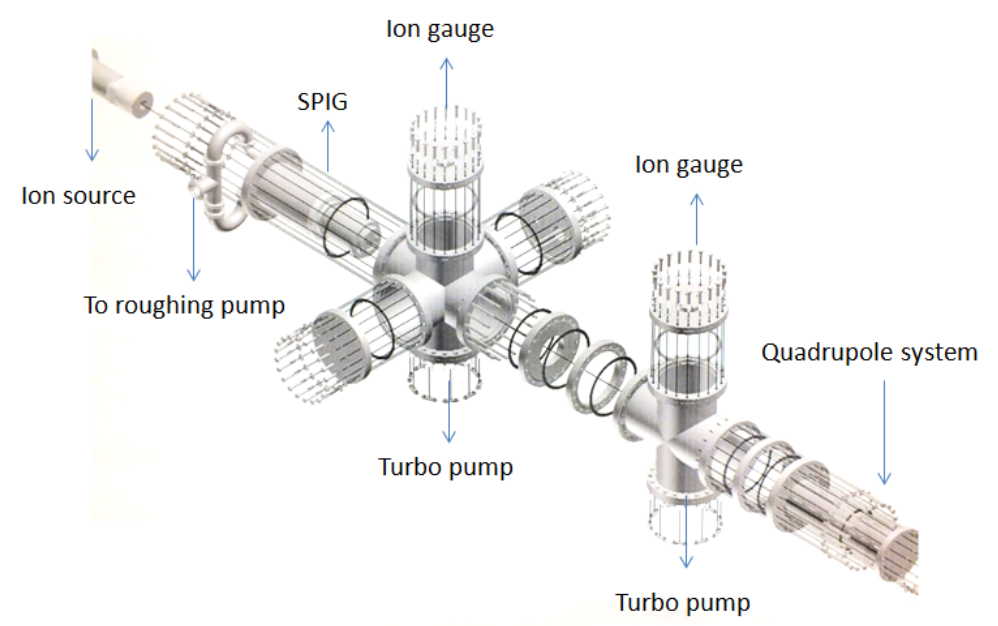

Figure 5.1: Schematics of the mass spectrometry system.

Ions are extracted from a barium acetate solution prepared in water and methanol using a nanoelectrospray source operated at relatively high pressures (between 0 and 15 psig). An electric field directs the ions through a $30 \mu m$ aperture towards an intermediate vacuum chamber where the SPIG resides. Ions are contained within the rods of the SPIG while gas flows away of the ion path under the influence of the pumps. The ion beam travels along the SPIG and exits into an Einzel lens that focuses the beam inside the quadrupole analyzer where the mass to charge ratio of the particle is determined and ions are counted.

\subsubsection{The Ion Source}

In order to test the barium tagging concept in gaseous xenon, a source of singly or doubly charged barium ions in high pressure gas is needed. Nanoelectrospray ionization was the technique chosen for a low flow, clean and efficient production of ions using minimum amounts of sample. An ion source using this concept was designed for the ion counting and extraction system for this work. 


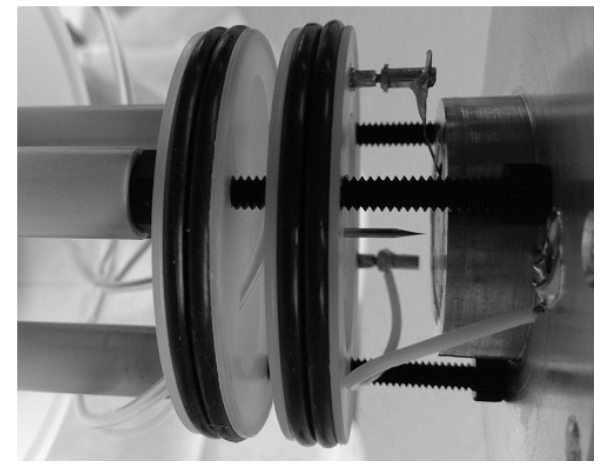

Figure 5.2: Close up view of the nanoelectrospray ion source mount.

Figure 5.2 shows a picture of the ion source setup. It consists of a teflon array holding a center-placed copper tube where the nanoelectrospray needle resides. The nebulising gas (nitrogen) is flowed to the tip of the emitter through a pair of stainless steel tubes to increase droplet fragmentation at a flow rate of $2 \mathrm{ml} / \mathrm{min}$. The emitter is placed at a fixed distance of $2 \mathrm{~mm}$ from the extractor electrode. Mechanical supports at both ends of the array prevent displacements of the source that could affect the geometry. The array resides in a 2.75 " canister that can be operated between 0 and $15 \mathrm{psig}$, and that holds the high voltage connections for the needle and extractor electrode. The source is removable for easy handling when nanoelectrospray emitter replacement is needed in between runs.

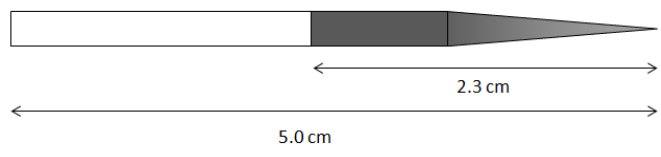

Figure 5.3: Diagram of glass nanoelectrospray emitter. Shaded area corresponds to the conductive coating.

The nanoelectrospray emitters used were $4 \mu \mathrm{m}$ tip borosilicate glass needles from New Objective, Inc. (Woburn, MA) with flow ranges between 40 and $100 \mathrm{nl} / \mathrm{min}$. The needles are $5 \mathrm{~cm}$ long with a conductive coating at the tip for electrical contact (see figure 5.3). Needles with tip openings of $1 \mu \mathrm{m}$ were initially tested but their use was discarded due to recurrent tip plugging affecting the spray modes and thus the operation of the system. The samples used were (1) Barium Acetate $\left(\mathrm{Ba}\left(\mathrm{C}_{2} \mathrm{H}_{3} \mathrm{O}_{2}\right)_{2}\right)$ at $10 \mathrm{mM}$ concentration in $20 \%$ methanol, (2) Cesium Iodide at $15 \mathrm{mM}$ in $20 \%$ 
methanol, $2 \%$ acetic acid, and (3) Rubidium Iodide at $50 \mathrm{mM}$ in $20 \%$ methanol. The last two were used mainly for calibration purposes due to their characteristic and easy identifiable mass peaks that cover most of the instrument's full mass range. The needles were loaded with 1-3 $\mu \mathrm{L}$ of sample using GELoader tips from Eppendorf.

The emitter electrode was operated at voltages between 0 and $2500 \mathrm{~V}$, with common threshold voltages (potential difference between emitter and extractor electrodes for which spray was induced) between 900 and $1300 \mathrm{~V}$. The electric current from the needle was monitored with a custom made nanoammeter coupled to the needle voltage circuit. The lifetime of the emitters when operating in stable conditions in cone-jet mode is $\sim 20 \mathrm{~h}$.

\subsubsection{Extractor Electrode}

In order to reduce gas flows into the system, small apertures on the extractor electrode are desired. The gas flow rate depends on the radius of the orifice as shown in equation (4.1). For nitrogen in the ion source vessel, with a pressure drop of 1 bar through an orifice of $15 \mu \mathrm{m}$, the expected flow rate is on the order of $\sim 20 \mathrm{ml} / \mathrm{min}$. This value scales up as the number of apertures increases. Extractor electrodes with different aperture distributions were manufactured and tested.

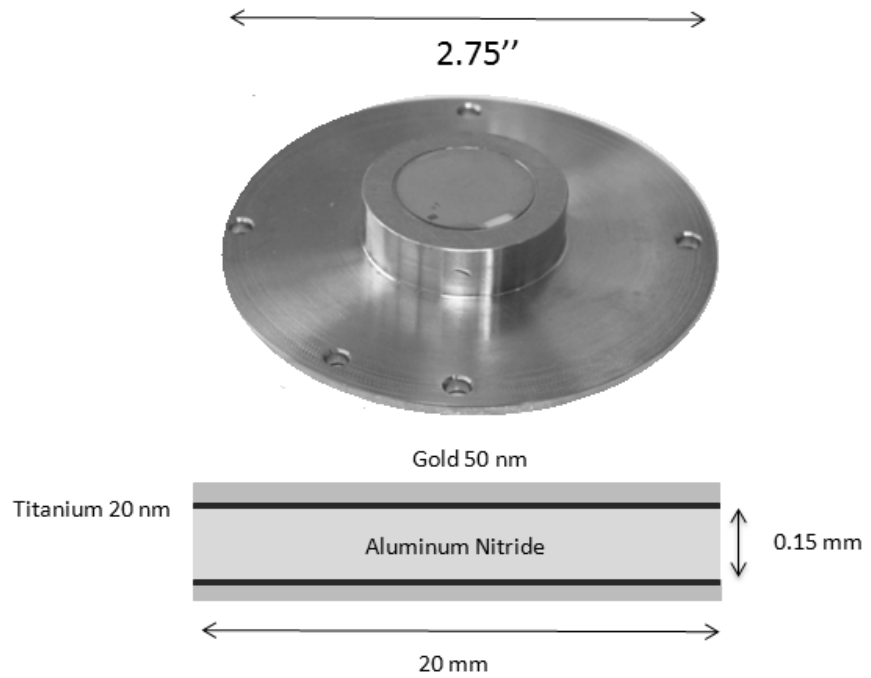

Figure 5.4: Picture of the extractor electrode with mounted ceramic nozzle. 
The extractor electrode consists of an aluminum nitride wafer of thickness 0.15 $\mathrm{mm}$ and diameter $20 \mathrm{~mm}$ purchased from Valley Design, Corp. (Shirley, MA). A 20 $\mathrm{nm}$ layer of gold was evaporated onto both sides of the disc and apertures on the order of $\sim 15 \mu \mathrm{m}$ radius were laser drilled by Oxford Laser Inc (Shirley, MA). The different patterns are shown in figure 5.5. The surfaces are electrically isolated and the disc is mounted in a stainless steel plate that resides at the end of the 2.75" canister. The effects of the geometry and the applied voltage across the electrodes were tested to optimize ion extraction into the system.
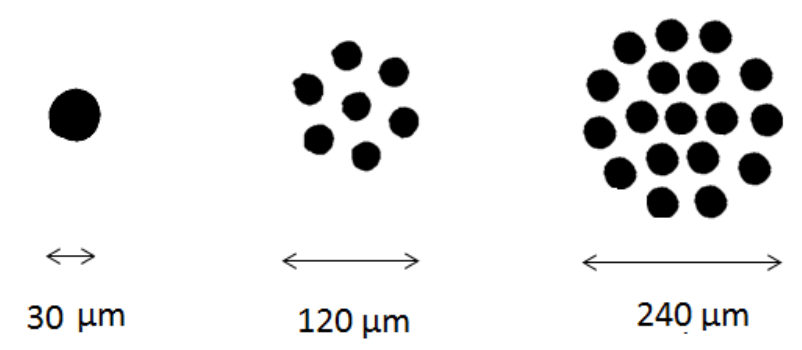

Figure 5.5: Diagram of extractor electrode aperture geometries.

The applied voltages to the ceramic were in the range of $0-200 \mathrm{~V}$. Current was monitored with a picoammeter coupled to the circuit. Voltages greater than $200 \mathrm{~V}$ applied on the extractor electrode were observed to cause gold etching on the surface. Salt depositions causing clogging of the apertures and around the spray zone were commonly observed to affect ion extraction efficiency. Due to needle-to-needle geometry variations, alignment issues were frequent; such problems were corrected by carefully choosing the needles with the most symmetric tips and by rotation of the needle on its axis until a signal was observed.

\subsubsection{SPIG}

The sextupole ion guide used for ion transport while residual gas was pumped out of the system is a device that belonged to a Waters Quattro II quadrupole-hexapolequadrupole mass spectrometer. The array is housed in a mount of rods that support two skimmer plates for ion focusing and kinetic energy control. A picture of the SPIG is shown in figure 5.6. 


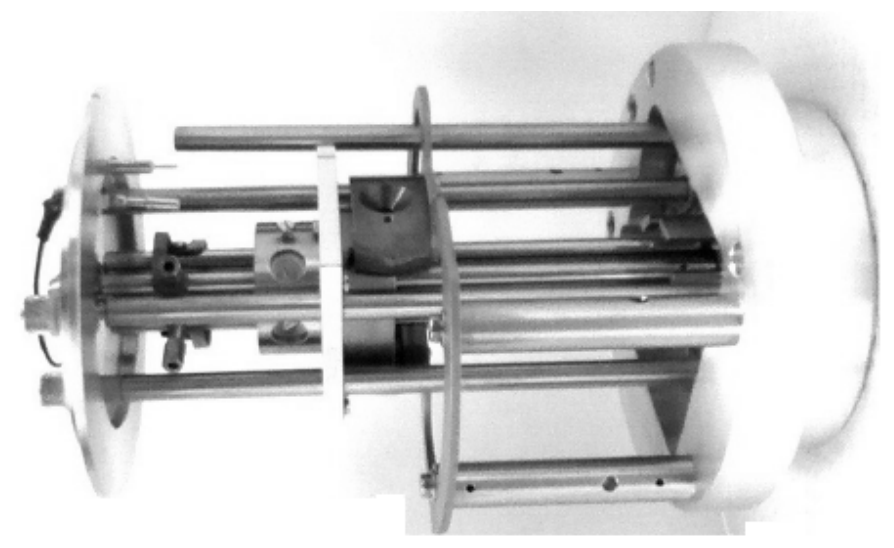

Figure 5.6: Picture of SPIG setup.

The SPIG is $14.37 \mathrm{~cm}$ long, with rods diameter of $0.50 \mathrm{~cm}$ with an inscribed radius of $r_{0}=0.475 \mathrm{~cm}$. The first skimmer aperture is $0.60 \mathrm{~cm}$ wide and the second one is $0.15 \mathrm{~cm}$. The exit aperture of the SPIG has $0.30 \mathrm{~cm}$ of diameter. The device operates at $1.6 \mathrm{MHz}$ with an RF voltage of $30 \mathrm{~V}$ peak-to-peak, no DC voltage is applied. The skimmer also plays the role of an ion optical lens, the first plate was operated between 0 and $200 \mathrm{~V}$ while the second one was held at voltages between 0 and $30 \mathrm{~V}$. The effect of the applied voltage to the skimmer was studied. The change in the RF voltage amplitude was observed to have no significant effect on the ion transport efficiency.

\subsubsection{Quadrupole Mass Analyzer}

The mass analysis and ion counting was performed using a quadrupole mass filter purchased from Extrel CMS, LLC (Pittsburgh, PA). The model is the MAX-500HT. It is coupled to an Einzel lens at its entrance for ion focusing into the filter. The rods are $19 \mathrm{~mm}$ in diameter and it operates at a frequency of $1.2 \mathrm{MHz}$. The analyzer scan mass range is between 1 and 500 mass units and it has expected transmission efficiency of $\sim 65 \%$. The detector is a low noise Channel Electron Multiplier with conversion dynode with $<90$ cps noise in positive ion counting mode. 


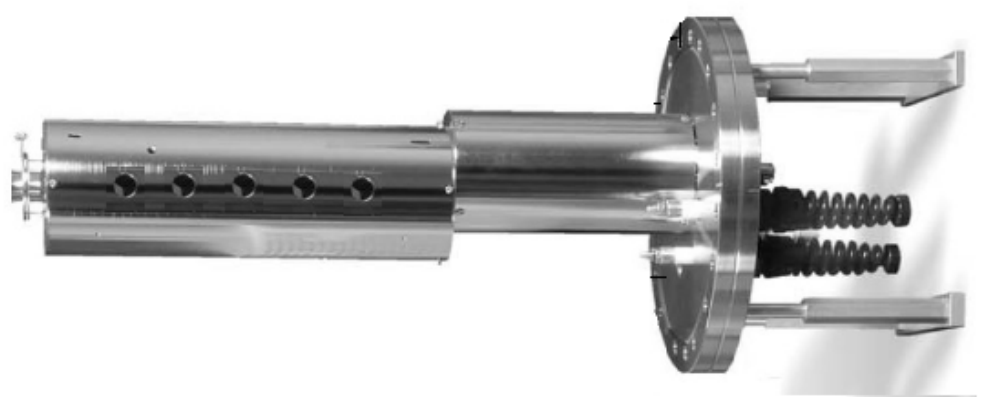

Figure 5.7: Quadrupole Mass Analyzer and CEM housing. The mount is a 6" CF.

The setup of the quadupole mass analyzer is shown in figure 5.7, the CEM is mounted off axis of the quadrupole to avoid background noise signals caused by discharge photons produced within the chamber. The data acquisition and analysis software used is the Merlin Automation Data System. All electronics, high voltage supplies and RF Oscillator connections are located at the end of the housing and supplied by the CS MS command system and QCi Quadrupole control modules also purchased from Extrel. The unit operates at pressures $<1 \times 10^{-5}$ mbar. The voltage specifications are listed in table 5.1.

\subsubsection{Summary of Operational Parameters for the System}

The voltage specifications for the electrodes in the system are listed in table 5.1. Potentials regarding the ion source and the SPIG configuration were floated to tune the system for maximum ion intensity. The RF and $\mathrm{DC}$ voltages for the quadrupole were automatically tuned by the software when a mass range was selected to scan, providing the best configuration for a stable trajectory of the selected ions within the analyzer. The multiplier and dynode electrodes were operated at the recommended values from the manufacturer. 


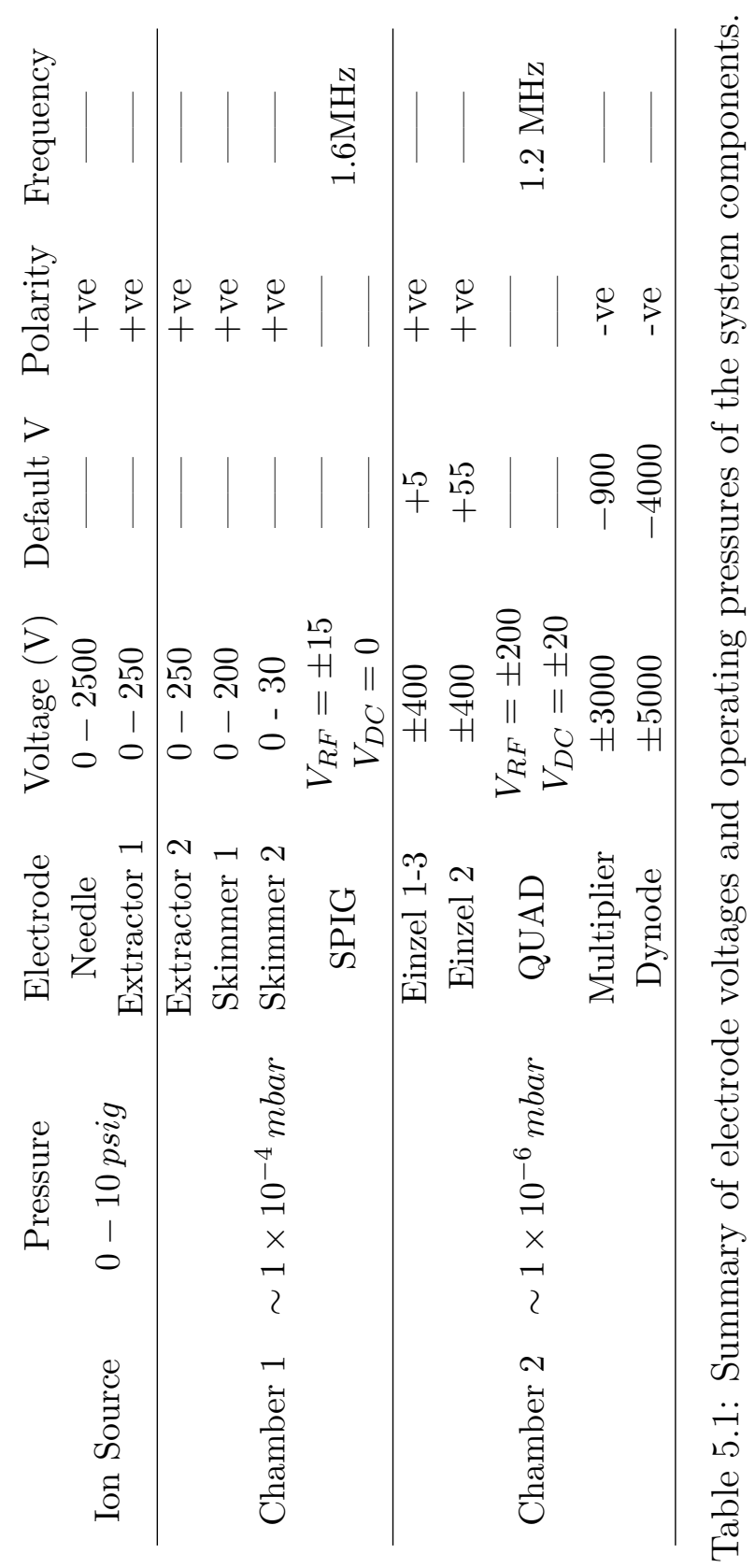




\subsection{SIMION Calculations}

Monte Carlo simulations using the SIMION 3D simulation software package were performed to acquire better understanding of the performance and properties of the system. They were used to calculate approximations of the ion transport efficiencies, to determine parameters such as electrode voltages to optimize ion transmission and to simulate the ion kinetic energies in the electrical field gradient of the ion path.

The SIMION framework uses the Runge-Kutta method to model ion trajectories under the influence of an electric field in a determined geometry and buffer gas pressure conditions. The whole ion extraction and counting system developed for this work was simulated in this framework with the voltage, frequency, and DC and RF amplitude parameters outlined in table 5.1. A schematic of the simulation for the whole system is shown in figure 5.8. Geometry parameters used are presented in table 5.2 .

The main limitation of this simulation was the inability to model the proper geometries of the nanoelectrospray emitter and the extractor electrode due to framework size constraints. SIMION's potential array memory limit is 50 million grid points requiring at least $400 \mathrm{MB}$ of $\mathrm{RAM}$ for the array. This is equivalent to a grid spacing limit of $0.10 \mathrm{~mm}$ in contrast to the emitter and extractor electrode aperture geometries in the $\mu \mathrm{m}$ scale. Skimmer electrode surface contours were approximated which also limited the accuracy of the calculated potentials at such instances of the ion path. 


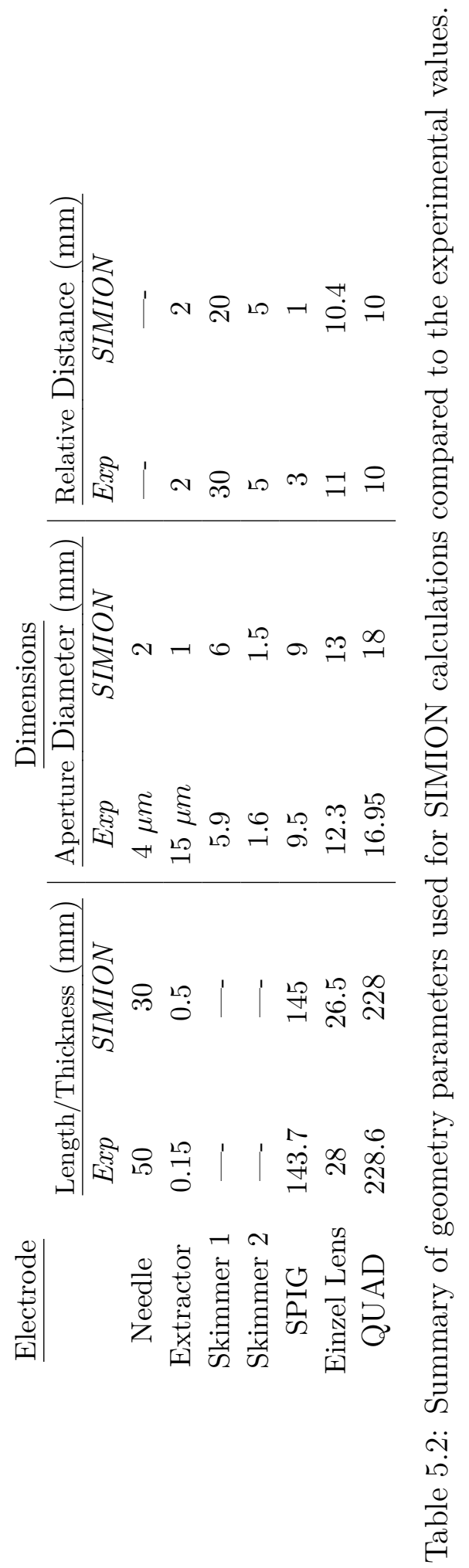




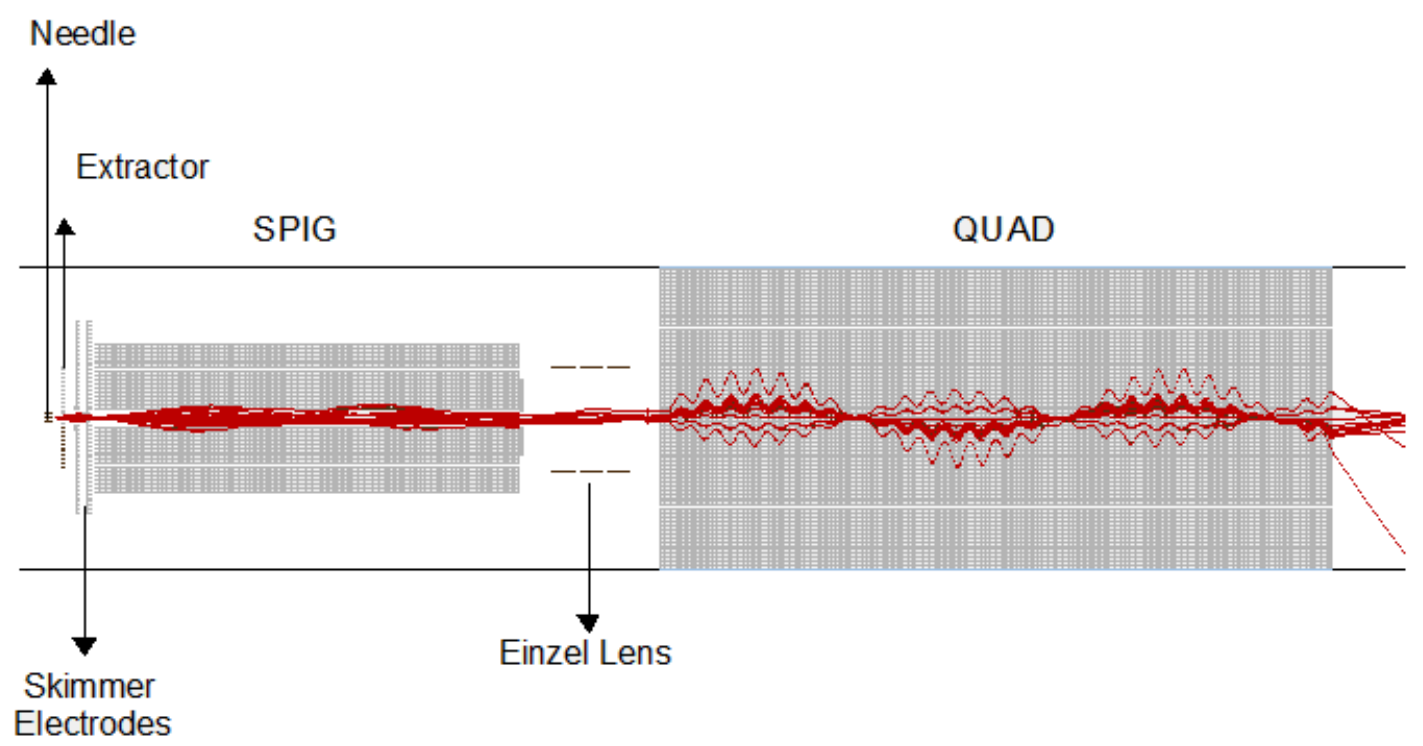

Figure 5.8: Schematic of the ion extraction and counting system simulation.

The effect of the nitrogen at pressures between 0 and 15 psig in the ion source was simulated using a modified version of the hard-sphere collision model of SIMION. This method treats the buffer gas as hard spheres that collide with the ions at different angles. The number of collisions depends on the input pressure, temperature and collision cross section parameters. The gas pressure and flow velocity were considered to be one dimensional in the direction of the ion path in an attempt to emulate the direction of the gas jet. The regions corresponding to the SPIG and quadrupole housings were simulated without the presence of a background gas.

Barium atoms of 136 amu at corresponding singly and doubly charged states were chosen as the test particles for positive ion transport calculations. A source of 1000 ions was randomly generated in a cone direction distribution with different semi vertical angles between 30 and $55^{\circ}$ to approach the Taylor cone mode formation. 


\subsubsection{Simulated Ion Extraction Efficiency}

The transmission efficiency across the extractor electrode was calculated with respect to variations in the ion emission angle and the ion source pressure. The model predicts that for higher emission angles the transmission efficiencies decrease, and as the pressure in the ion source increases, the transmission increases. This behaviour is explained by the fact that the ion motion gets constrained by the collisions with the buffer gas allowing them to be more efficiently guided by the electric field through the extractor aperture. Figure 5.9 shows the calculated transmission efficiencies of the extractor electrode with a $100 \mathrm{~V}$ potential drop across for different pressures at various ion emission angles. Such potential drop is presented as it corresponds to operational experimental settings at which ion intensities were maximized.

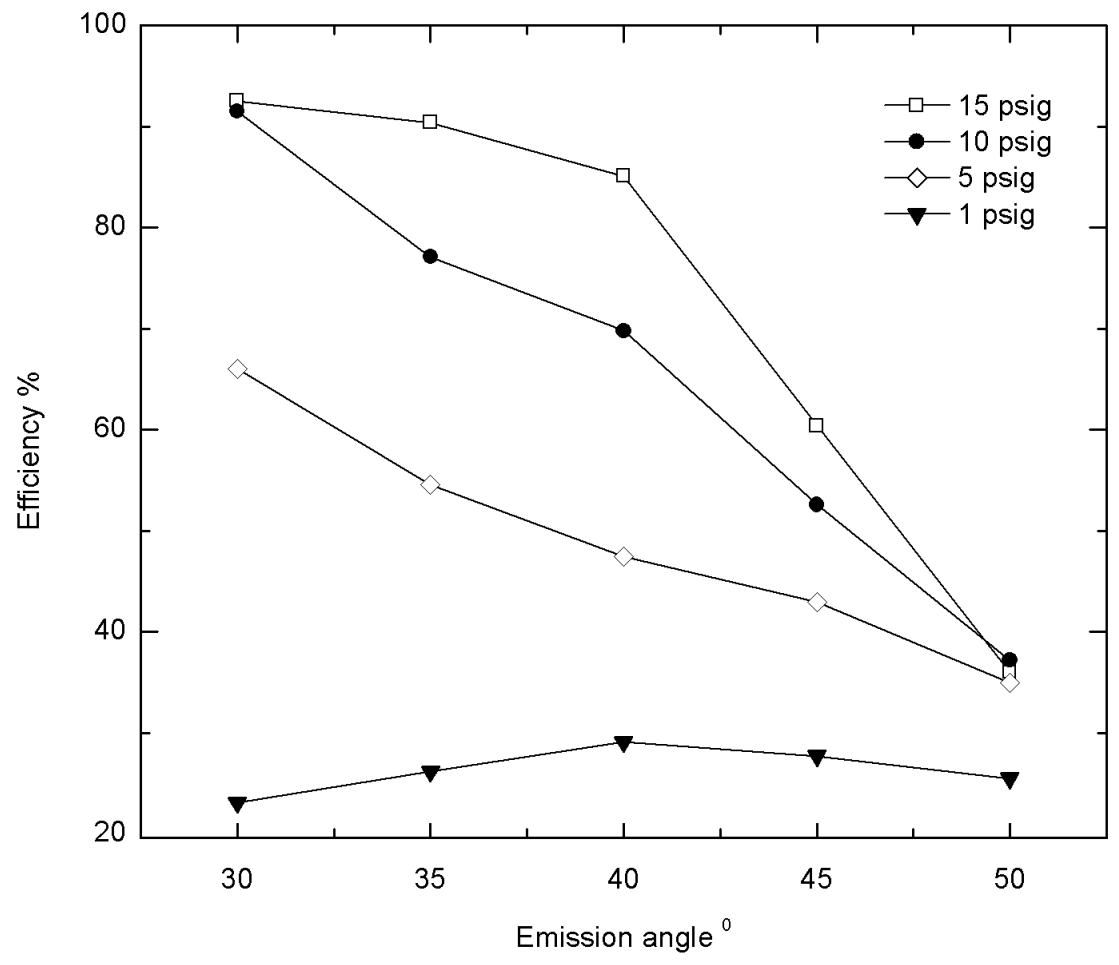

Figure 5.9: Simulated transmission efficiencies for barium ions of 136 amu at different emission angles and operating pressures with $100 \mathrm{~V}$ potential drop across the extractor electrode. 
From the ion trajectory simulations, low ion source pressures cause unstable trajectories for light particles when they collide with the buffer gas as the linear momentum transfer deviates them from the confinement region. At high ion source pressures, the ion-gas collision chances increase and thus light ions are more likely to maintain a narrow trajectory yielding better transmittance across the aperture. Figure 5.9 confirms such behaviour and predicts that as the initial ion spread angle increases, ion losses are more prompt because the electric field is not strong enough to guide them through the aperture.

Considering that the aperture dimensions are almost three orders of magnitude higher than the experimental case, much lower efficiencies in the built setup are expected. Moreover, solvent evaporation, possible molecule formation and other ion interactions in the solution are not taken into consideration.

\subsubsection{Ion Kinetic Energies}

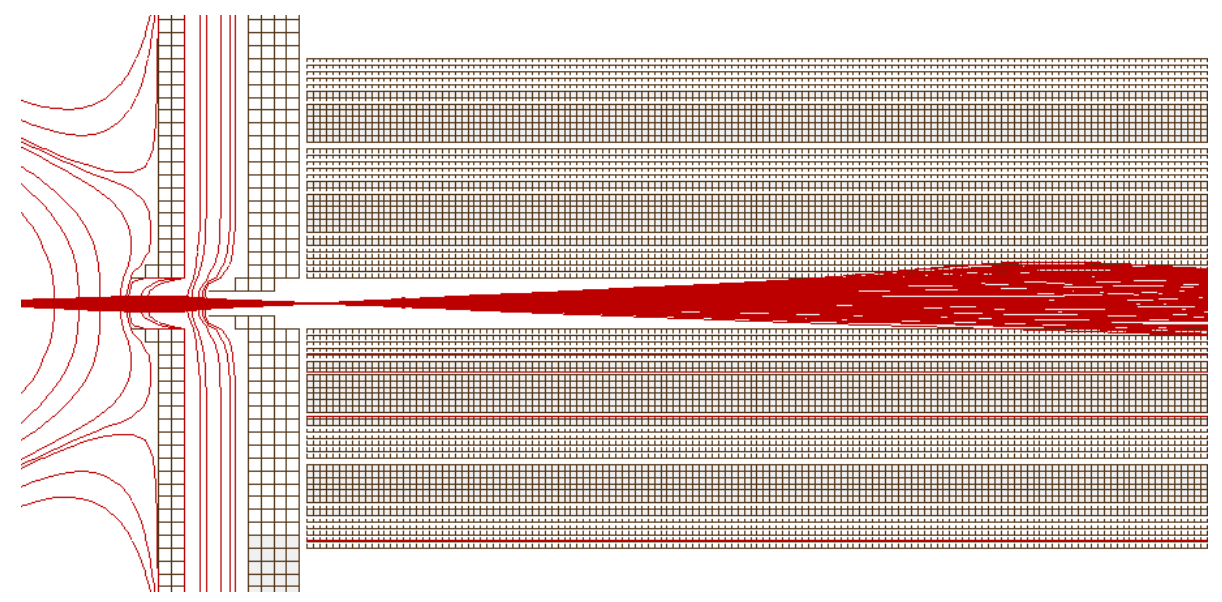

Figure 5.10: Simulation results for ion trajectories across the skimmer plate at the entrance of the SPIG. Electric field lines in the region between the electrodes are shown. Ions are singly charged, with mass of $136 \mathrm{amu}$ and the potential drop within the skimmer is $60 \mathrm{~V}$.

Skimmer electrode plates are commonly used in mass spectrometry to couple the exit of the extractor electrodes to the entrance of sextupole guides. Their main purpose in this system is to contribute to the removal of the residual gas coming from the ion 
source and to help in the ion focusing at the entrance of the SPIG. Ion trajectory simulation results are shown in figure 5.10. It can be observed that the beam profile is changed across the conical shaped skimmer plates providing an ion focusing for better transmittance within the SPIG.

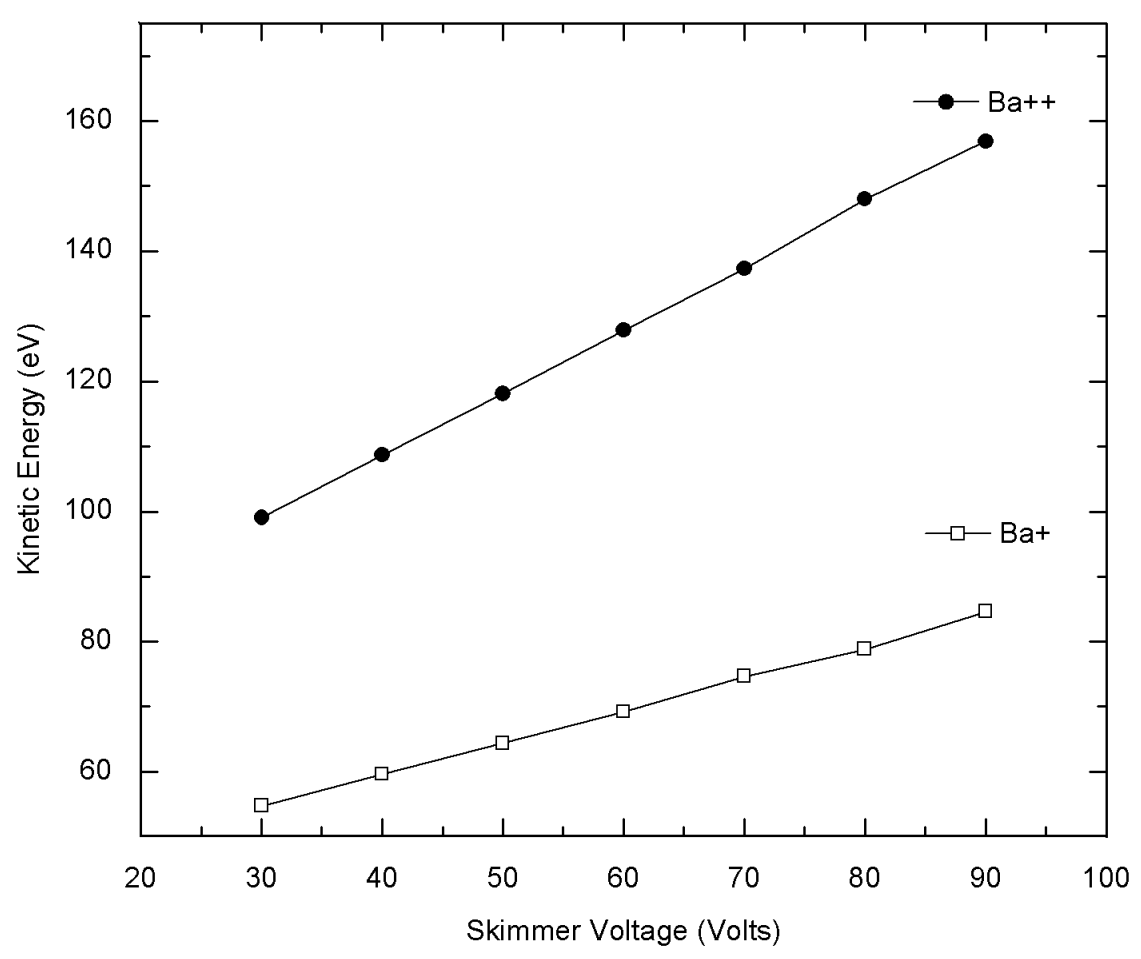

Figure 5.11: Calculated ion kinetic energies with respect to skimmer voltages for $\mathrm{Ba}^{+}$and $\mathrm{Ba}^{++}$ions. Uncertainties on kinetic energies are in the order of $\sim 10 \mathrm{eV}$ from the statistical variation of the energy spread.

Ion kinetic energies depend on the ion mass and the velocity of transport. Results from the simulation shown in figure 5.11 evidence the effect of the skimmer applied voltage on the ion energies. Ions get accelerated through the optical path at higher skimmer voltages. Low incident beam energy spreads can then be achieved by applying lower potentials on the cone electrodes. Ion stability and transmittance within the sextupole guide under different kinetic energy schemes didn't present any 
variation in this particular model.

\subsubsection{Einzel Lens Distance Optimization}

Another important tool for ion focusing in the system is the Einzel lens configuration. As described previously in section 4.2.1.1, these arrays of cylindrical electrodes do not affect the energy of the incoming ions and are of great advantage for SPIG Quadrupole couplings. Ion trajectories for the lens were calculated and are presented in figure 5.12.

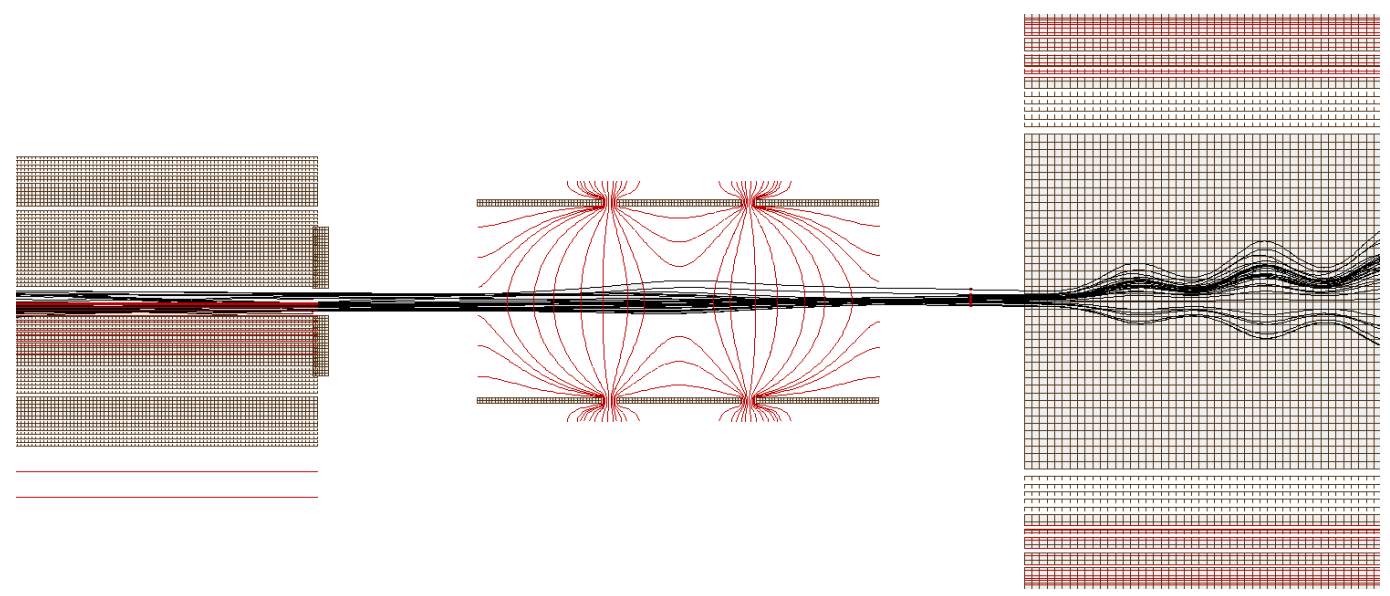

Figure 5.12: Simulation results for ion trajectories across the Einzel lens that focuses the ions into the quadrupole analyzer. Field lines in the array are drawn. Flying ions are singly charged particles of $\mathrm{m}=136 \mathrm{amu}$ with kinetic energies of $\sim 60 \mathrm{eV}$. SPIG - Einzel distance is $10 \mathrm{~mm}$.

In the Einzel lens configuration ions get electrostatically accelerated and subsequently decelerated causing unaltered exit-entrance ion kinetic energies. The beam shape of the ions can be modified by changing the applied electrode voltages and the provided focusing depends on the array geometry. As part of the design of the ion counting system, SIMION calculations were used to determine the relative position of the Einzel lens and the SPIG exit to provide the best achievable transport efficiency of the ions based on the fixed geometry of the Einzel lens-quadrupole configuration provided by the manufacturer. Results for different electrode voltages are shown in figure 5.13 . 


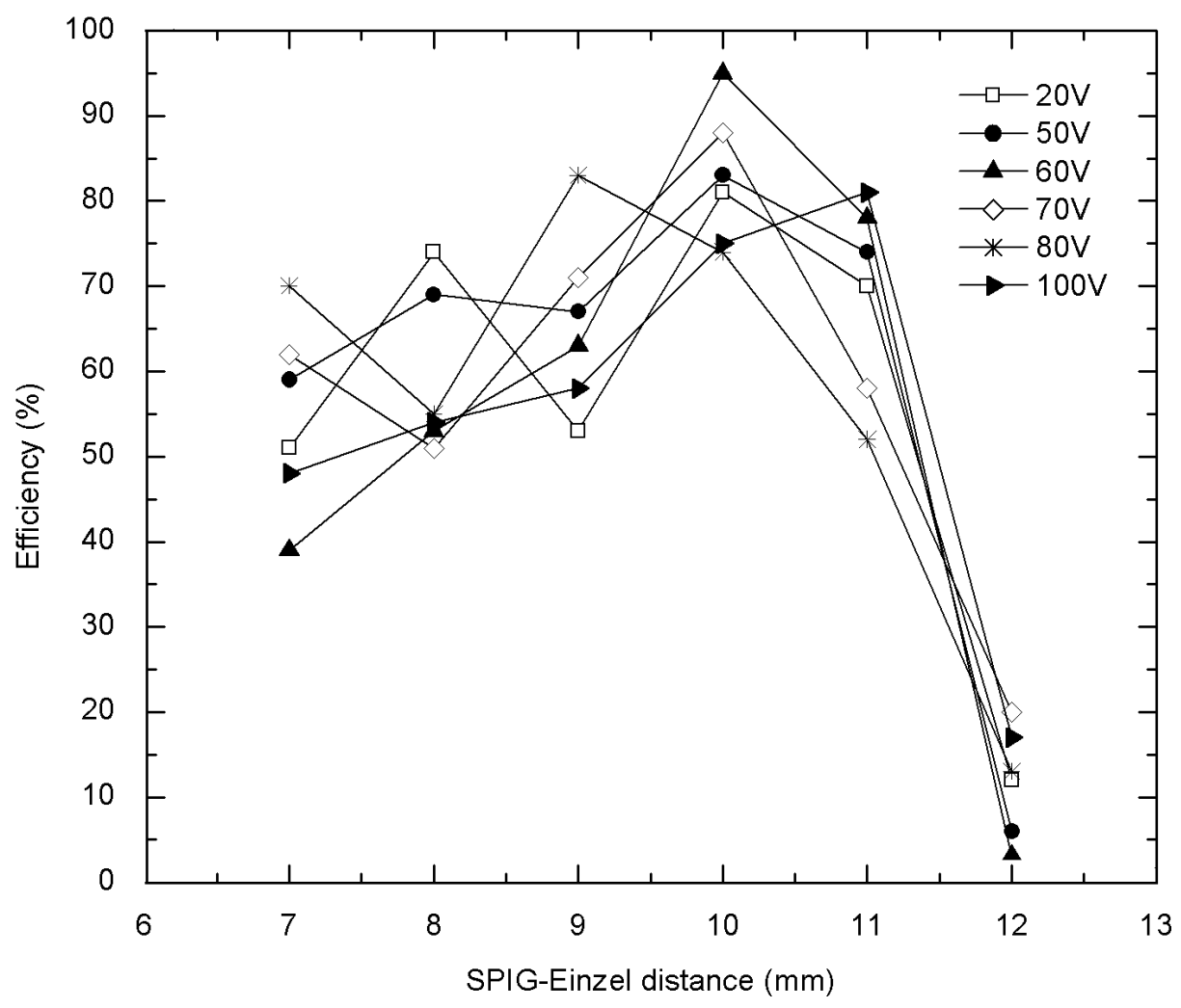

Figure 5.13: Einzel lens parameter optimization for efficient ion transport between the SPIG exit and Quadrupole entrance.

In the model used, efficiencies greater than $70 \%$ were obtained for a SPIG-Einzel Lens distance of $10 \mathrm{~mm}$. The focal point at voltages between $50 \mathrm{~V}$ and $60 \mathrm{~V}$ was located at the entrance of the quadrupole preventing ion losses at that stage due to ion profile geometrical constraints. Because the focusing voltage depends linearly on the beam energy, Einzel lenses with high entrance beam energies require higher operating voltages to achieve comparable efficiencies. 


\subsection{Experimental Results}

In order to test the feasibility of the proposed barium ion extraction and counting system for the barium tagging facility for the Enriched Xenon Observatory, the described setup was assembled and commissioned for continuous operation. A series of experiments was carried out to optimize the system and characterize its components. The accomplished results are described below.

\subsubsection{Spraying Characteristics}

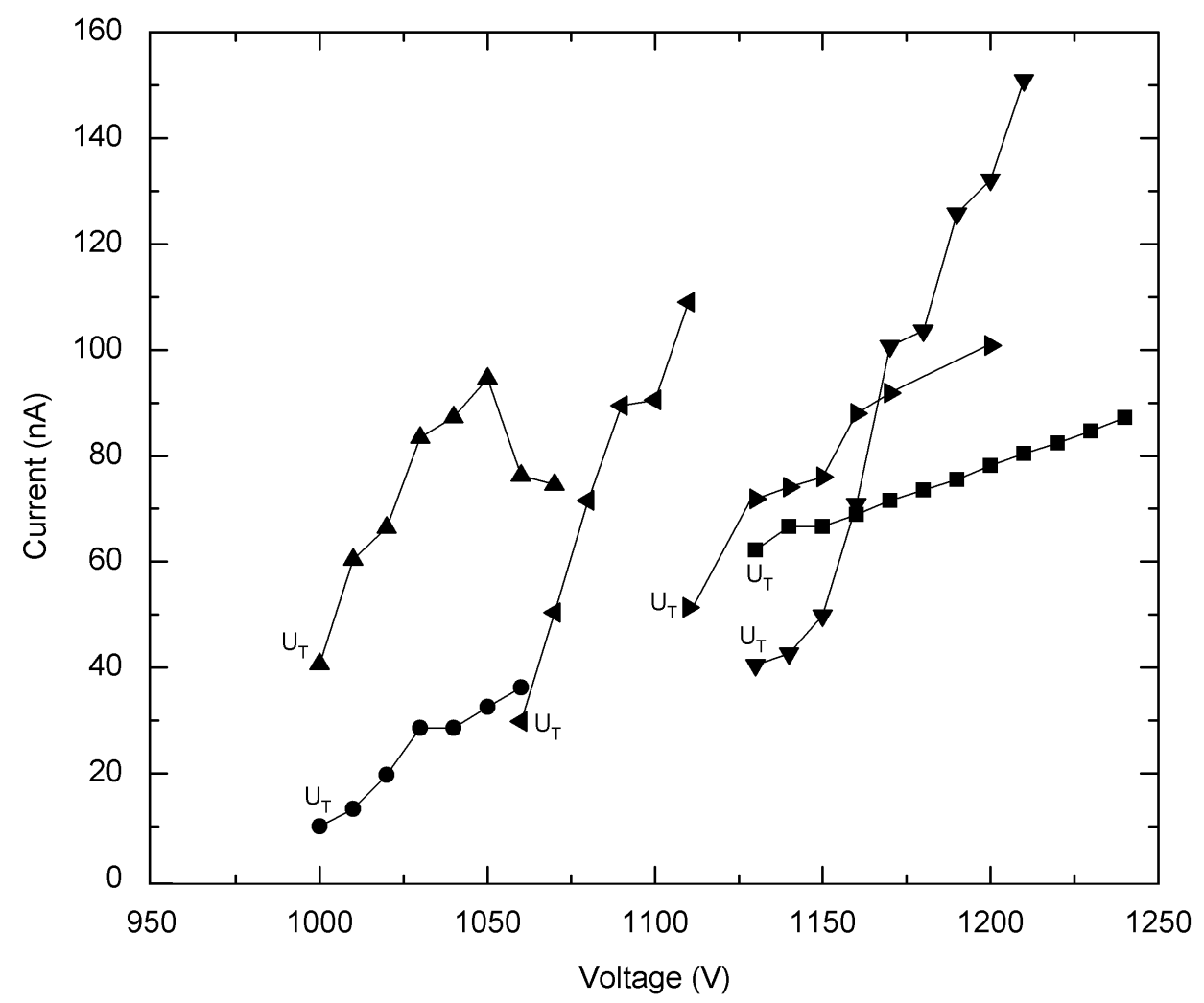

Figure 5.14: Variation in voltage and spray current for a set of needles. Each set of connected points represents a different needle. The threshold voltage, $U_{T}$, is indicated for every case. The corresponding experimental uncertainties for current and voltage are $\pm 5 \mathrm{nA}$ and $\pm 2 \mathrm{~V}$. 
The nanoelectrospray emitters used were characterized according to their currentvoltage relation, from which spray modes were identified for the determination of the stable cone-jet mode operation. Such region of stability, as well as the corresponding threshold spray voltage, varied from needle to needle. These changes can be observed in figure 5.14, which shows the applied voltages and corresponding spray currents for a random set of needles. It can be observed that the threshold voltage at which the emitter starts spraying varies from needle to needle. Such characteristics were found to be very sensitive to the initial geometry of the needle tip. Variations in the initial geometry are attributed to both manufacturing and handling processes, as well as salt deposits on the needle tip during operation.

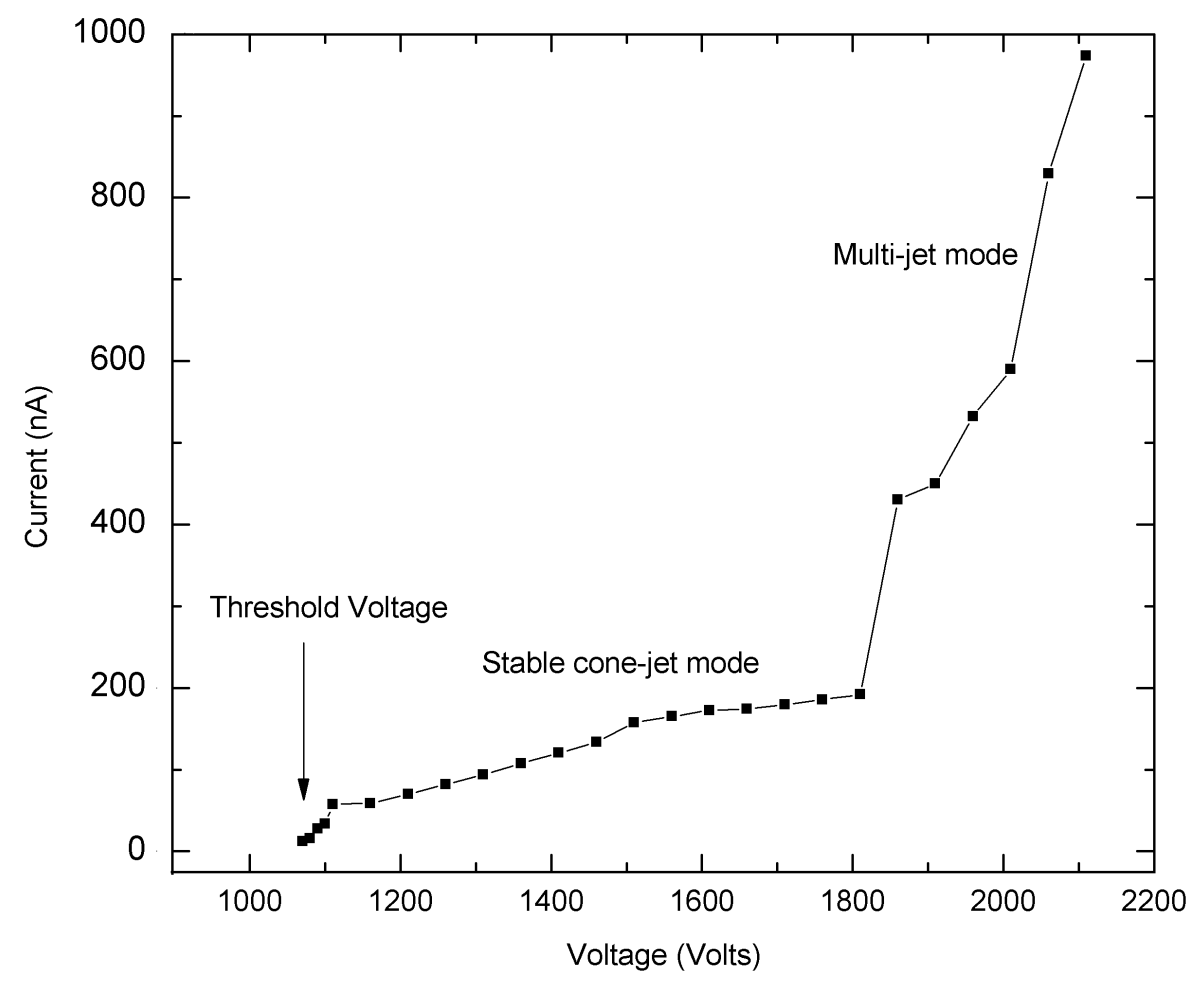

Figure 5.15: Spray mode identification from current voltage relation on nanoelectrospray emitters. The corresponding experimental uncertainties for current and voltage are $\pm 5 \mathrm{nA}$ and $\pm 2 \mathrm{~V}$.

Figure 5.15 shows an example of the most common spray behaviour for selected 
emitters with almost identical geometries used for stable nanoelectrospray operation. From the figure the different spraying modes described in the previous chapter can be identified and are shown to be dependent on the applied voltages. For such particular needle a stable region between $1200 \mathrm{~V}$ and $1800 \mathrm{~V}$ is observed, with a threshold voltage at $\sim 1100 \mathrm{~V}$ in accordance to the theoretical prediction for an emitter with the given dimensions.

\subsubsection{System Calibration}

The calibration of the mass spectrometer was performed using CsI, RbI and Barium Acetate solutions. The corresponding expected and measured mass values are listed in table 5.3. The advantages of the first two were the characteristic single and double peaks that allowed easy identification in the spectrum. For the latter due to multiple ion fragmentations a greater amount of peaks are obtained in neighbouring regions of the spectrum making the identification more difficult, and therefore only barium monoacetate peaks, which have a very recognizable pattern due to the isotopic barium content, were used for calibration purposes.

\begin{tabular}{|c|c|c|c|}
\hline Ion & Expected $\mathrm{m} / \mathrm{z}$ & Calibrated $\mathrm{m} / \mathrm{z}$ & Relative abundance \\
\hline \hline${ }^{85} \mathrm{Rb}^{+}$ & 84.91 & 84.91 & $72.2 \%$ \\
\hline${ }^{87} \mathrm{Rb}^{+}$ & 86.90 & 86.91 & $27.8 \%$ \\
\hline${ }^{133} \mathrm{Cs}^{+}$ & 132.85 & 132.90 & $100 \%$ \\
\hline${ }^{138} \mathrm{Ba}\left(\mathrm{CH}_{3} \mathrm{COO}\right)^{+}$ & 197.22 & 197.20 & \\
\hline
\end{tabular}

Table 5.3: Spectrum peaks of CsI, RbI and Barium Acetate used for calibration of the mass spectrometer.

The chosen peaks provide a reliable calibration in the 1-200 amu mass range. Although the instrument has a detection range up to $500 \mathrm{amu}$, such interval contains the operation regions of most interest and is adequate for the desired purposes. The accuracy of the mass measurement is around $0.01 \%$ for different runs. 


\subsubsection{Typical Barium Acetate Fragmentation}

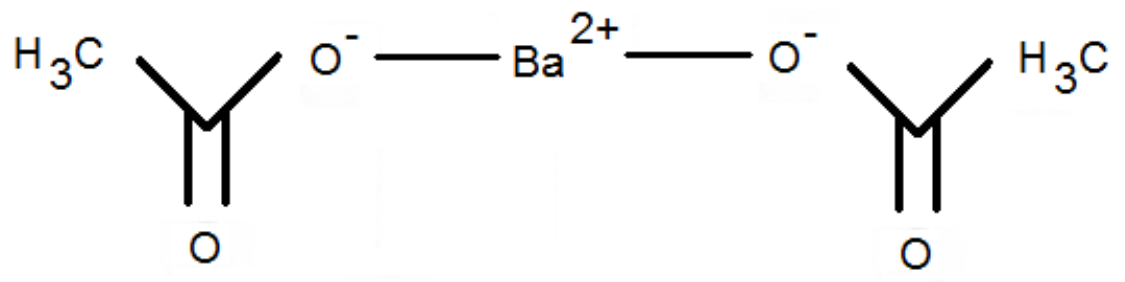

Figure 5.16: Diagram of barium acetate molecule bonding.

Barium acetate is composed by a doubly charged barium atom bonded to $\mathrm{C}$, O, and $\mathrm{H}$ atoms as shown in figure 5.16. Possible molecule separation in the solvent before the process and ion collisions in the high field between the needle and the extractor electrode induce ion dissociation. Different fragmentation products are observed depending on the bond strength, on the applied electric fields and on the interactions with the surrounding water, methanol and nitrogen in the ion source setup. Table 5.4 lists the masses for different expected compounds in the barium acetate dissociation.

\begin{tabular}{|c|c|c|}
\hline Compound & Name & Mass (amu) \\
\hline \hline $\mathrm{Ba}\left(\mathrm{CH}_{3} \mathrm{COO}\right)_{2}$ & Barium (di) acetate & 255.42 \\
\hline $\mathrm{Ba}\left(\mathrm{CH}_{3} \mathrm{COO}\right)$ & Barium (mono) acetate & 196.67 \\
\hline $\mathrm{Ba}\left(\mathrm{CH}_{3}\right)$ & Barium (mono) methyl & 152.95 \\
\hline $\mathrm{Ba}(\mathrm{OH})$ & Barium Hydroxide & 154.91 \\
\hline $\mathrm{Ba}\left(\mathrm{CH}_{3} \mathrm{O}\right)$ & Barium Methoxy & 168.94 \\
\hline $\mathrm{Ba}^{+}$ & Singly charged Barium & 137.905 \\
\hline $\mathrm{Ba}^{++}$ & Doubly charged Barium & 68.95 \\
\hline
\end{tabular}

Table 5.4: Possible fragmentations of Barium Acetate

The typical mass spectrum for barium acetate dissociation is shown in figure 5.17. The peaks were identified using the information in table 5.4. 


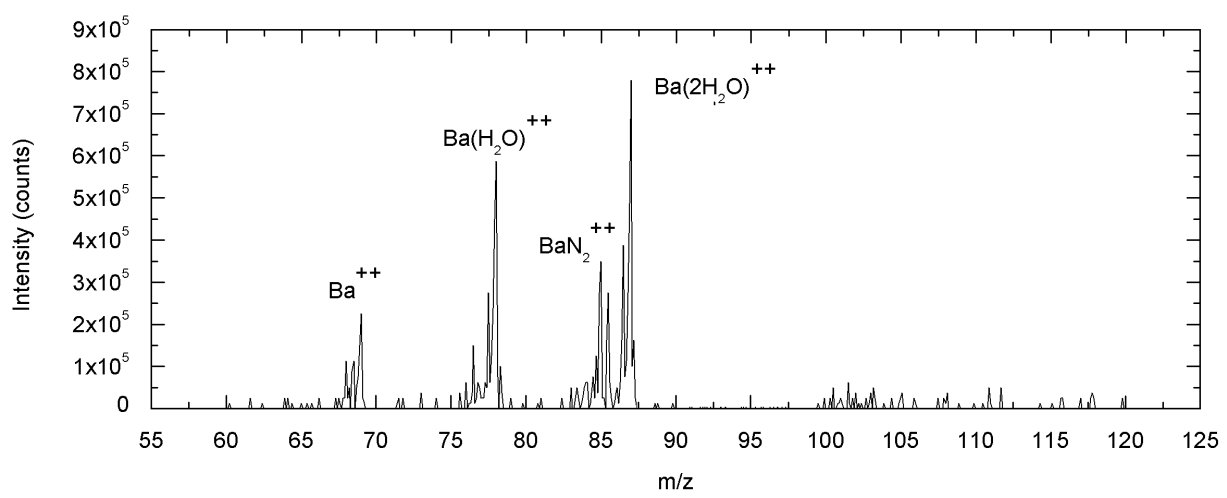

(a)

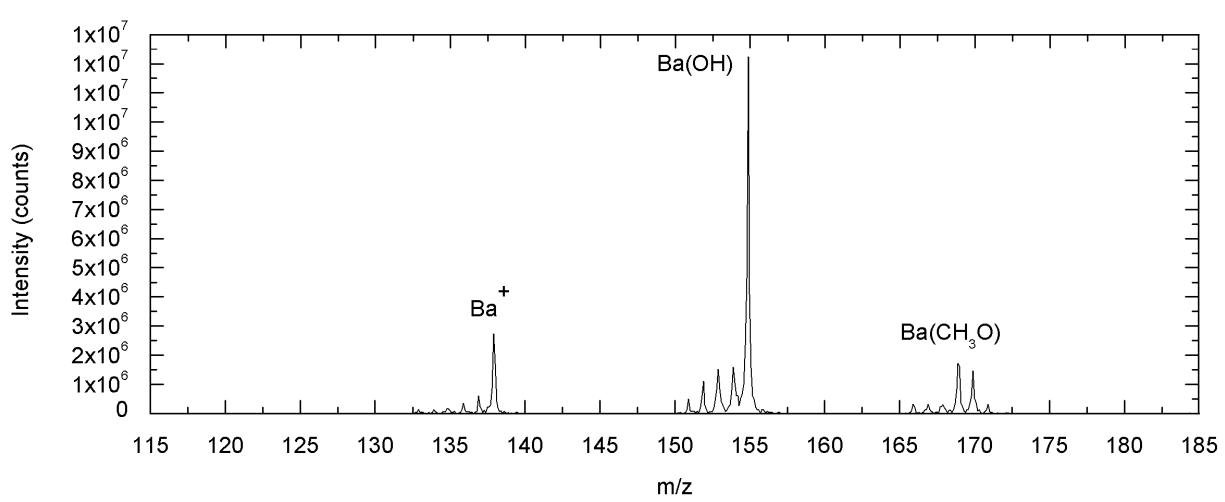

(b)

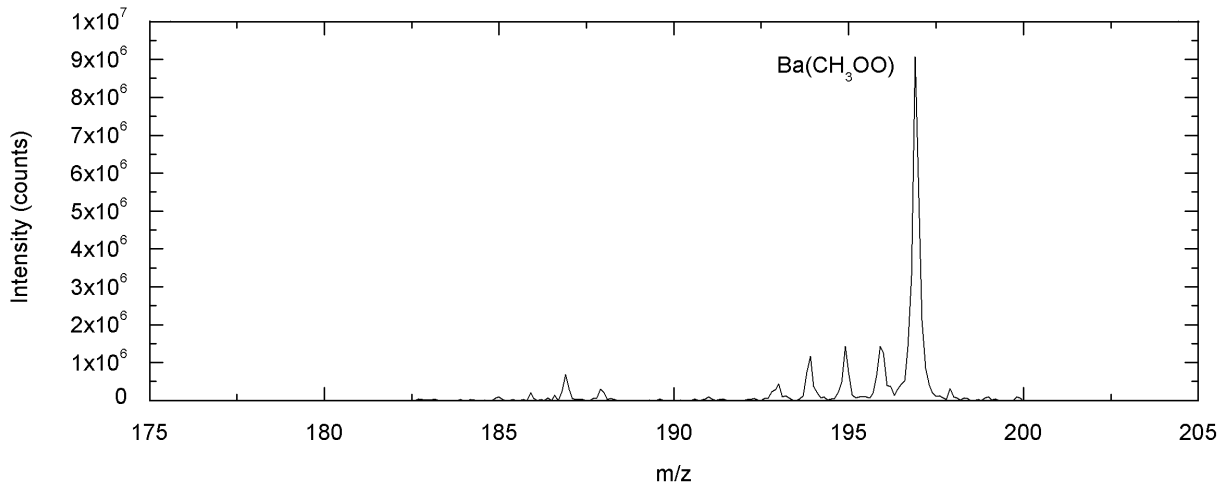

(c)

Figure 5.17: Mass spectrum of barium mono-acetate dissociation at $100 \mathrm{nA}$ of spraying current, $70 \mathrm{~V}$ and $20 \mathrm{~V}$ drop across the extractor electrode and skimmer respectively and ion source operating at $3 \mathrm{psig}$ in nitrogen at $1 \mathrm{ml} / \mathrm{min}$ flow rate. Scan ranges from 60 to $120 \mathrm{~m} / \mathrm{z}$ (a), 120 to $180 \mathrm{~m} / \mathrm{z}$ (b) and 180 to $200 \mathrm{~m} / \mathrm{z}$ (c) are shown. 
An important characteristic of barium compounds is the presence of the five contiguous peaks of increasing intensity with increasing mass. These represent the isotopic content of barium at the corresponding abundances listed in table 5.5. The spectra obtained for both singly and doubly charged cases are shown in figure 5.18.

\begin{tabular}{|c|c|c|}
\hline Isotope & Mass(amu) & Abundance (\%) \\
\hline \hline${ }^{138} \mathrm{Ba}$ & 137.905 & 71.7 \\
\hline${ }^{137} \mathrm{Ba}$ & 136.905 & 11.23 \\
\hline${ }^{136} \mathrm{Ba}$ & 135.904 & 7.9 \\
\hline${ }^{135} \mathrm{Ba}$ & 134.905 & 6.6 \\
\hline${ }^{134} \mathrm{Ba}$ & 133.904 & 2.4 \\
\hline${ }^{132} \mathrm{Ba}$ & 131.905 & 0.1 \\
\hline${ }^{130} \mathrm{Ba}$ & 129.906 & 0.1 \\
\hline
\end{tabular}

Table 5.5: Stable barium isotopes with corresponding masses and abundances.

The mass analysis and discrimination performed with the quadrupole mass analyzer is accomplished by measuring the mass to charge ratio of the detected ions. It can be observed from the corresponding spectra for the barium ion at the two different charged states in fig 5.18 that the mass peak separation for the singly charged case is 1 mass unit; accordingly, for the doubly charged case the mass separation is half a mass unit. An excellent mass resolution is then required for observation and identification of doubly charged barium peaks, as obtained in this case. 

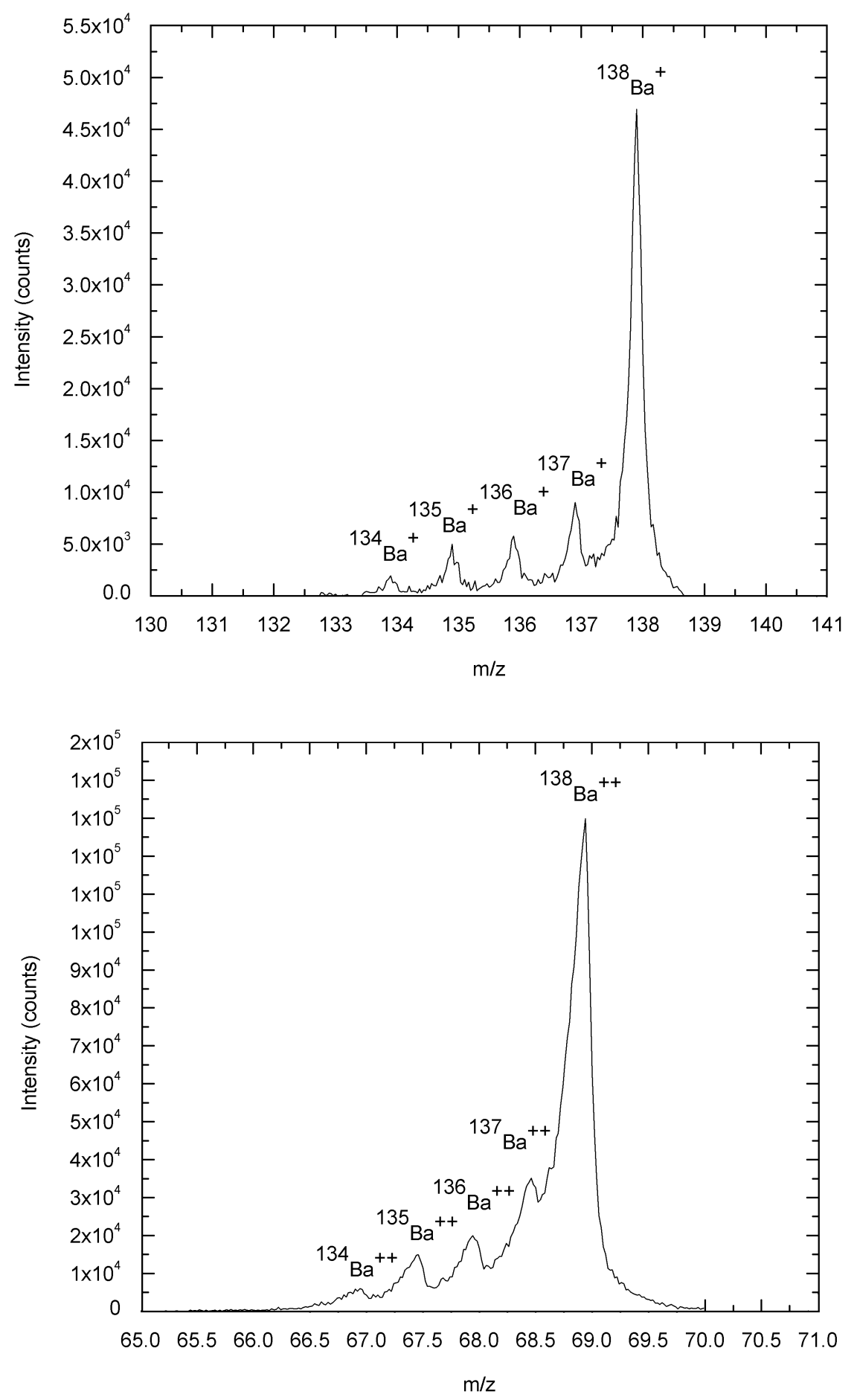

Figure 5.18: Mass spectrum of barium (top) singly and (bottom) doubly charged obtained with the nanoelectrospray ion source. Uncertainties on intensities for this particular case are not significant to show as the variations are mainly dependent on reproducibility constraints. 


\subsubsection{Skimmer Electrode Effect on $\mathrm{Ba}^{+}$and $\mathrm{Ba}^{++}$Detection}

An important effect on the production of singly and doubly charged barium ions was observed when varying the skimmer electrode voltages at the entrance of the SPIG. The ion acceleration suffered at this stage of the ion path and its expected effect in the kinetic energy from the calculations, showed an increase of detection intensity of $\mathrm{Ba}^{++}$for higher skimmer potentials. Figure 5.20 illustrates such configurations.

The obtained relation between the ion intensities in the detector with respect to the skimmer voltage variation is shown in figure 5.19. It is observed that for higher potentials doubly charged ions are more prompt to be transferred along the system.

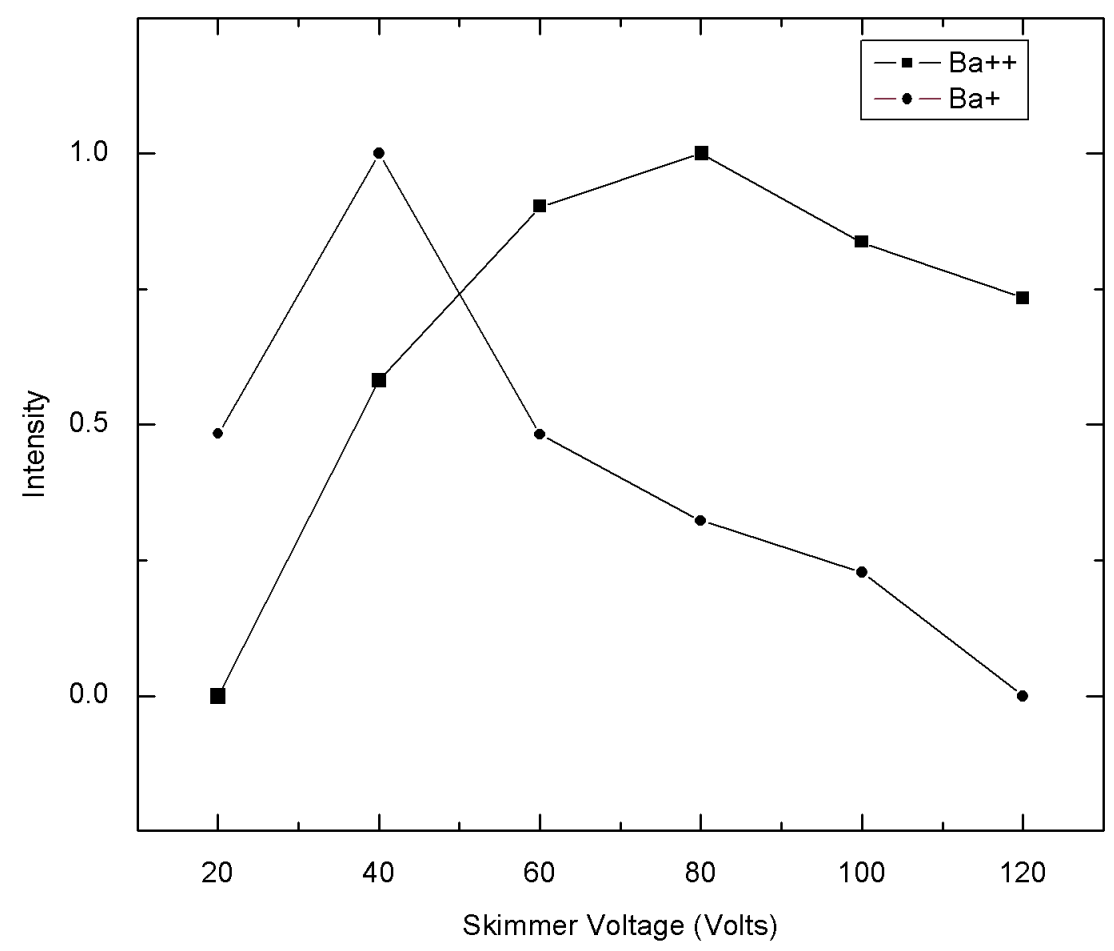

Figure 5.19: Skimmer voltage effect on $\mathrm{Ba}^{+}$and $\mathrm{Ba}^{++}$normalized ion intensities. Variation in the voltage reading is of $\pm 2 \mathrm{~V}$.

The ability to select between charged states of barium on the basis of changes in 


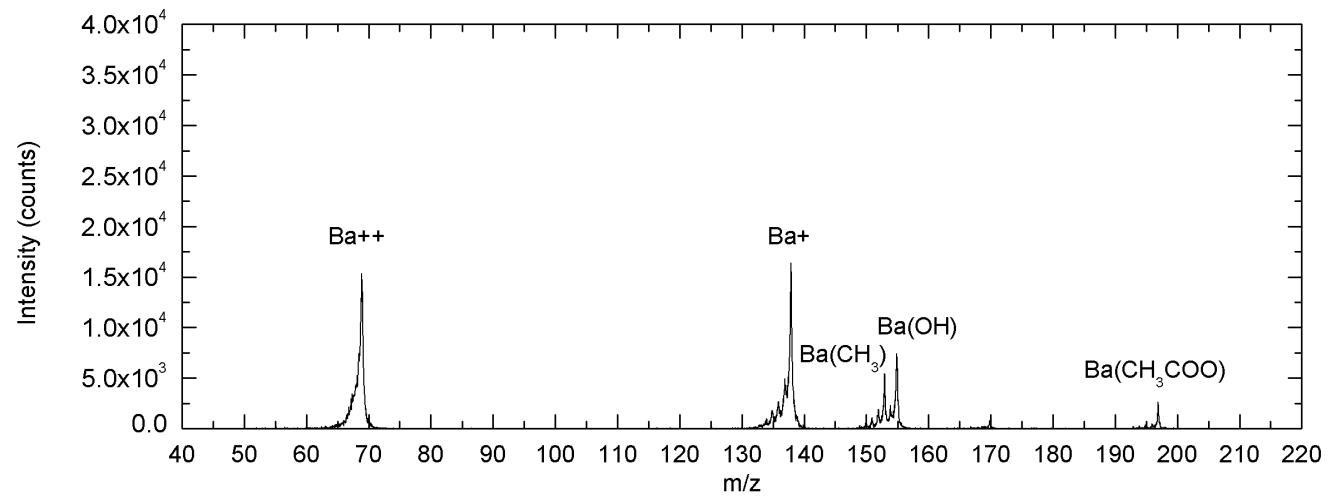

(a)

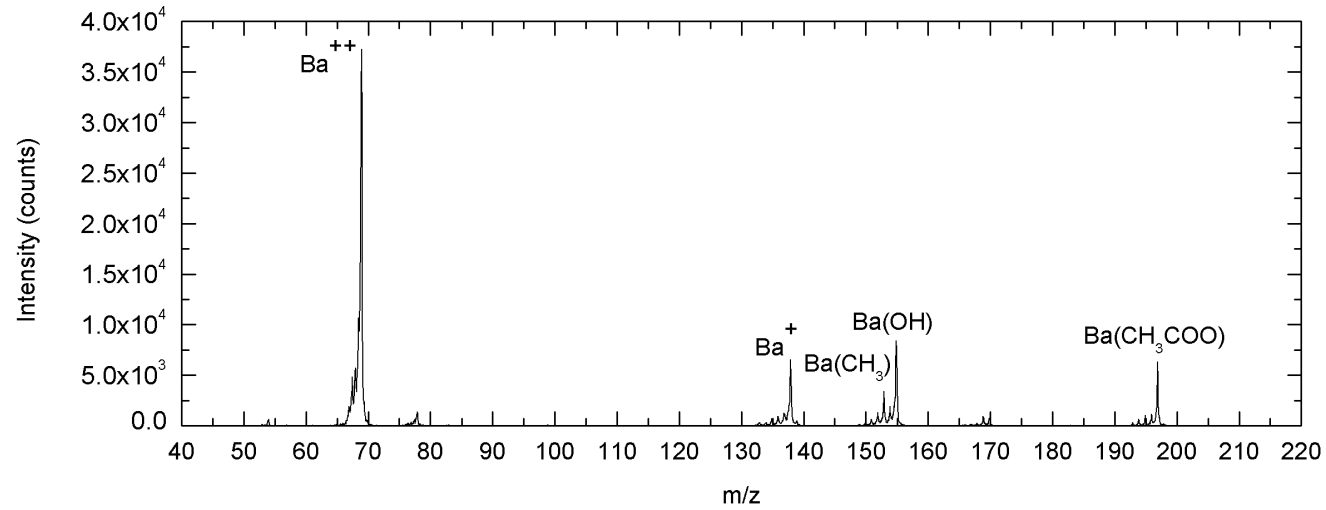

(b)

Figure 5.20: Selection ability of singly or doubly charged barium ions for variations in the skimmer potential. (a) Skimmer potential $=70 \mathrm{~V}$, (b) Skimmer potential $=$ $140 \mathrm{~V}$ 
kinetic energies by the skimmer potential can be of great utility in the performance tests of the ion trapping and laser identification states of the process as no need for a charge conversion state is needed. However, in the full working system such charge conversion state is absolutely necessary because in the neutrinoless double beta decay scenario, only doubly charged barium ions are produced.

\subsubsection{Ion Source Pressure and Geometry Effects on Ion In- tensity}

The geometry effects on the extractor aperture were tested at different electric field and pressure conditions. Figure 5.21 and figure 5.22 show the results obtained.

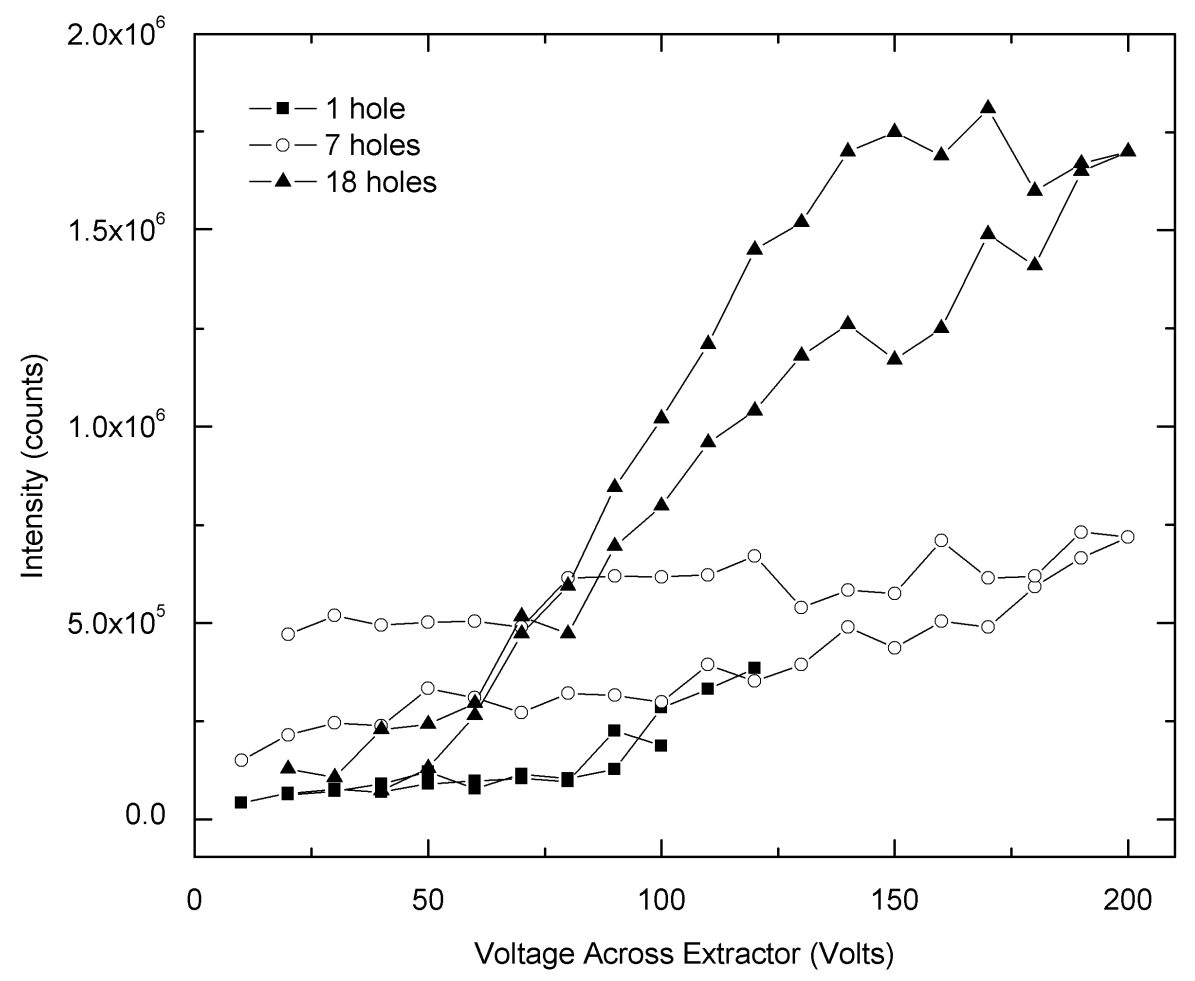

Figure 5.21: $\mathrm{Ba}^{++}$counts for different potential drops across the extractor electrode for different aperture geometries. Intensity uncertainties are not significant to show as the variations are mainly dependent on reproducibility constraints. The voltage readings have corresponding uncertainties of $\pm 2 \mathrm{~V}$. 
As expected from the model calculations higher aperture diameters provide better extraction efficiencies that can be observed from the increase in ion detection in the analyzer. Although the effect of high extractor electrode potentials are favorable, degradation on the surfaces of the gold plated electrode were observed for voltages greater than $100 \mathrm{~V}$. Continuous gold etching on the surfaces of the extractor causing discontinuities in the superficial electrical contact, were responsible for reproducibility issues after several runs. Salt depositions inducing clogging of the extractor electrode and emitter apertures were common. Such a situation yielded reduced extraction diameters and multi-jet spraying modes impacting ion intensities at the end of the system.

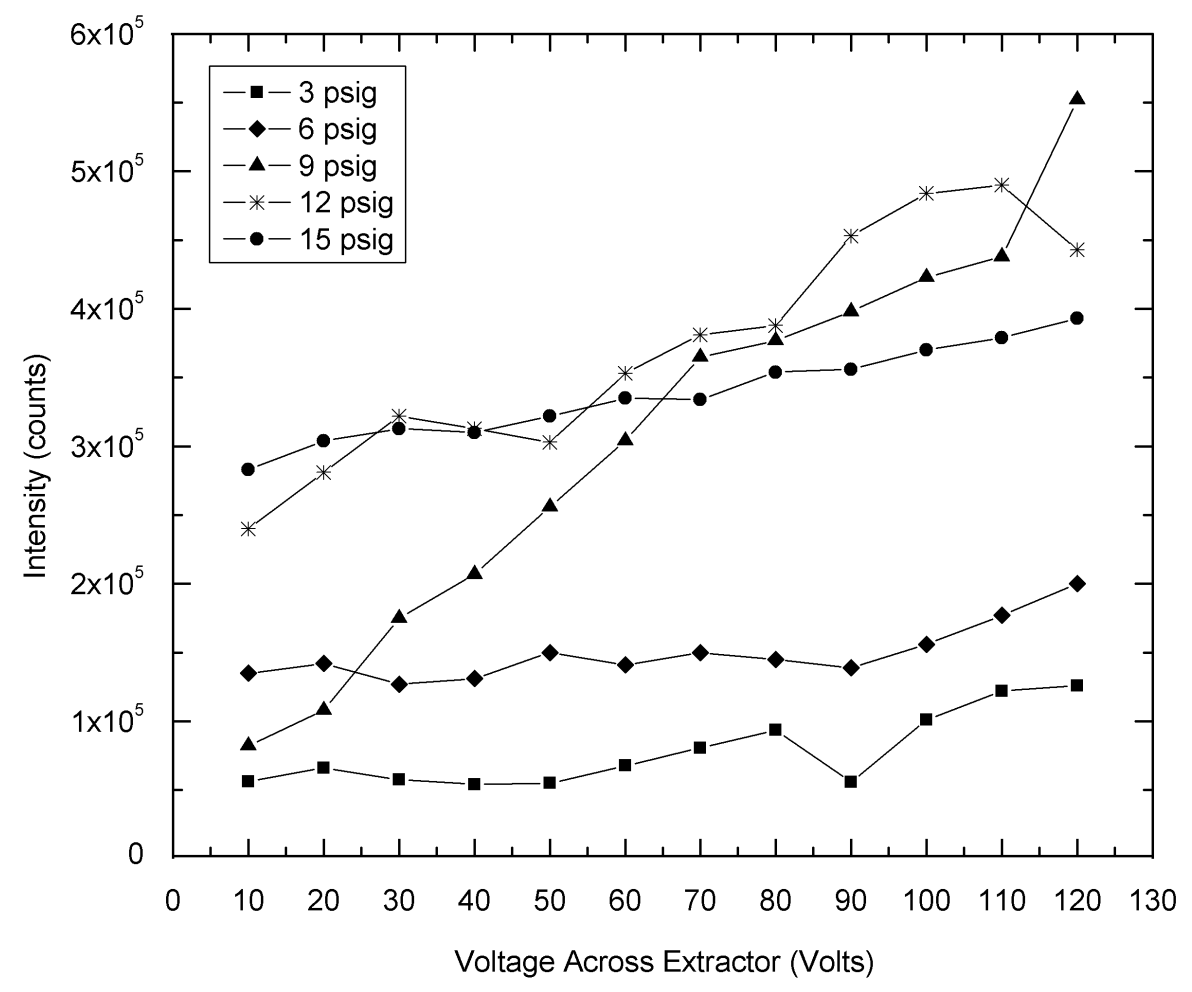

Figure 5.22: $\mathrm{Ba}^{++}$counts for different potential drops across the extractor electrode for different ion source operating pressures. Intensity uncertainties are not significant to show as the variations are mainly dependent on reproducibility constraints. The voltage readings have corresponding uncertainties of $\pm 2 \mathrm{~V}$. 
Higher pressures had a positive effect on ion extraction and ion intensity detection. By operating the ion source at higher pressures nanoelectrospray extraction efficiencies are expected to increase due to the reduction of surface tension around the molecules. Such behaviour is also expected from the ion trajectory calculations that evidence more stable ion trajectories through the extractor electrode when particles collided with the buffer gas.

\subsubsection{Efficiency, Resolution and Stability}

Efficiencies in the system are difficult to determine as no exact number of ions entering the system from the sprayed solution is known. An approximation of the transmission efficiency at different stages of the electric field gradient was achieved measuring ion currents at the electrodes. Although this approach helped in the identification of the areas of most significant ion losses, it is not a reliable method to determine absolute efficiencies as the measured currents include contributions from salt or buffer ions and ionization from electrical discharges or electrochemical interactions along the ion path. 


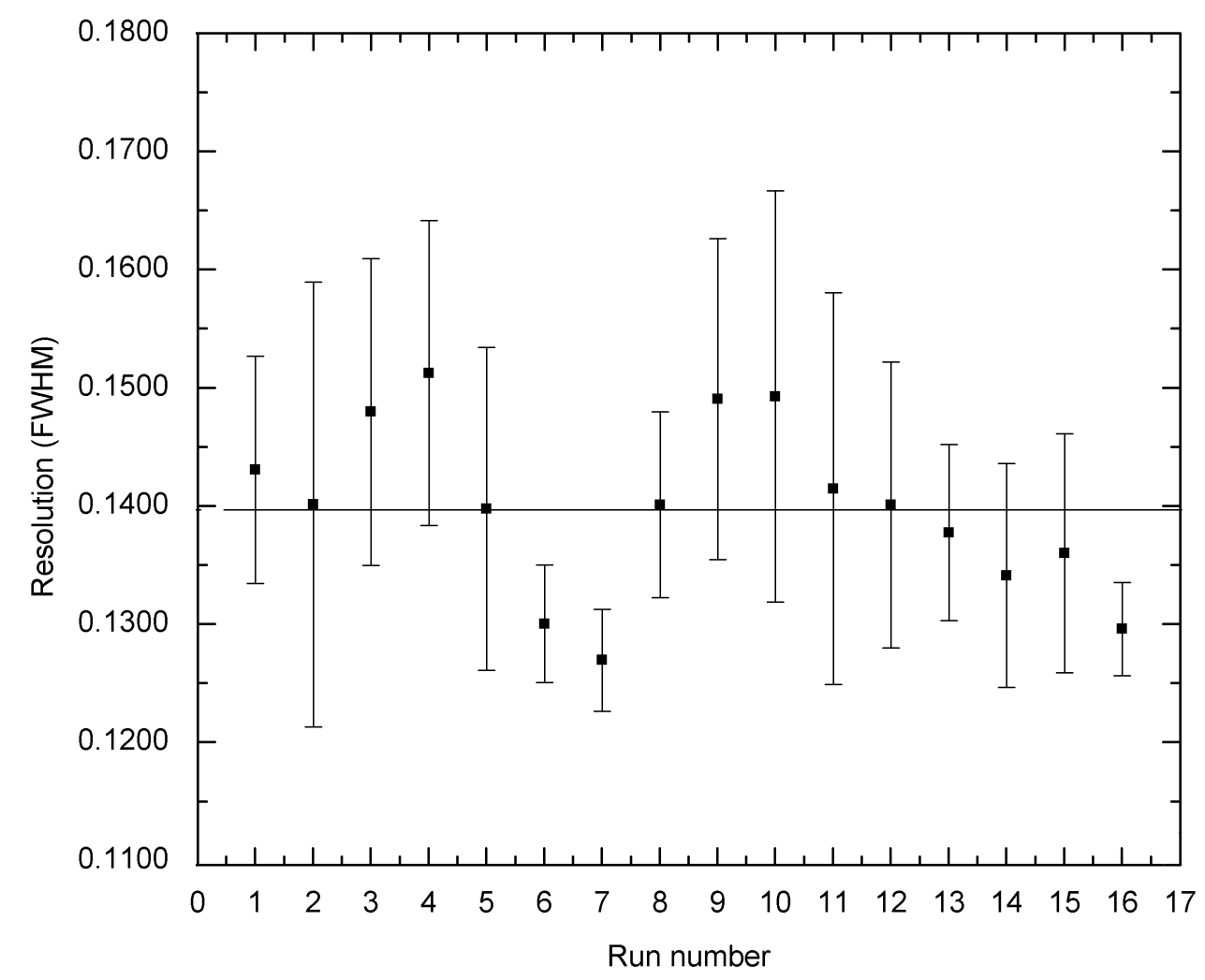

Figure 5.23: Average resolution of the mass analyzer for $\mathrm{Ba}^{++}$detection for different runs.

The average resolution of the system for the $\mathrm{Ba}^{++}$ion in different runs is shown in figure 5.23. These values were found to be consistent and didn't depend on external factors. The values presented were obtained by calculating the FWHM of the mass peaks after approximating them to a Gaussian distribution. Such resolutions in conjunction with the accuracy of the calibration are ideal for the identification of unknown compounds in the system. 


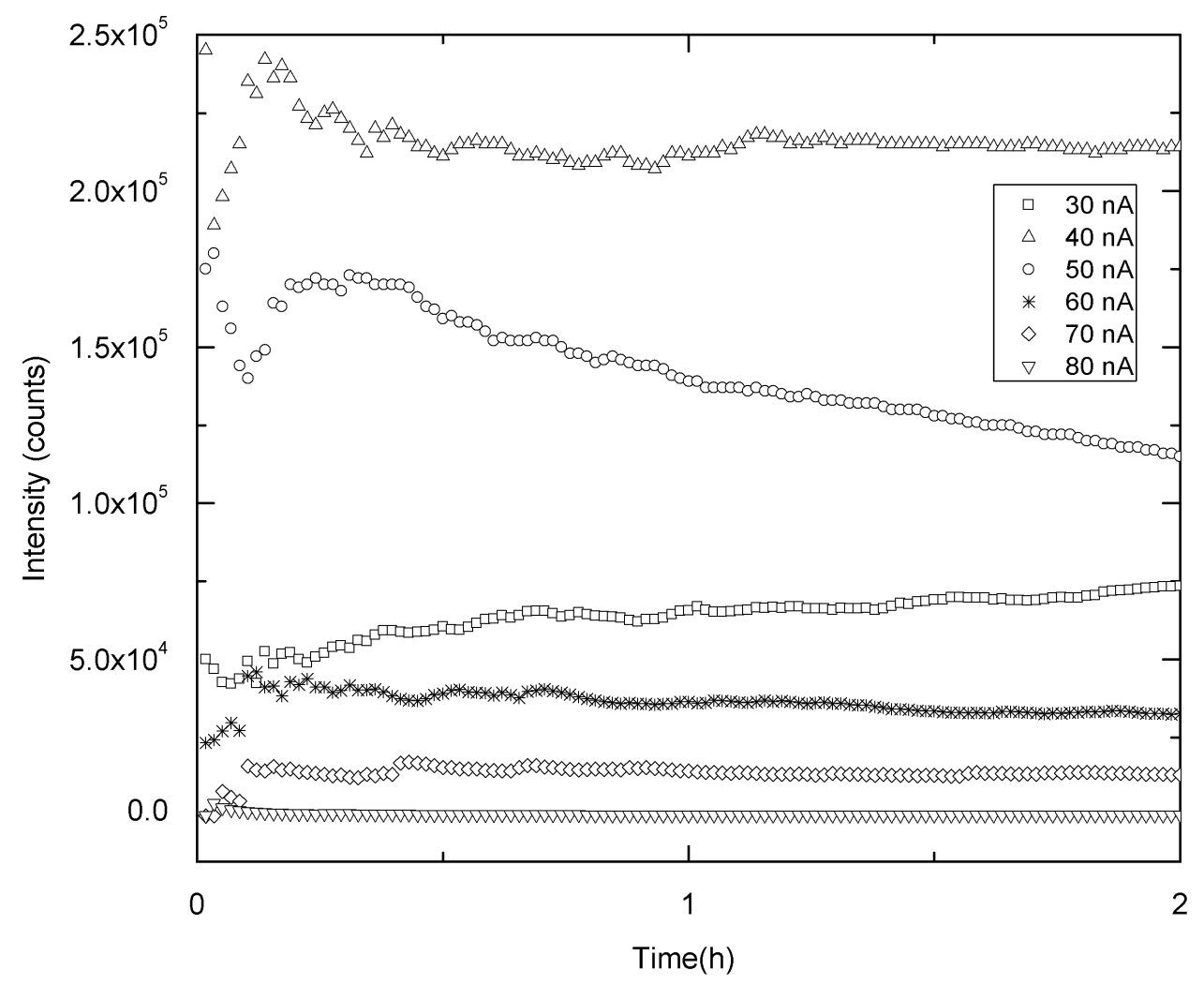

Figure 5.24: Ion production stability over $2 \mathrm{~h}$ long runs at different operating pressures of the ion source.

Needle to needle geometry variations beyond experimental control had a negative impact on the reproducibility of the ion intensities for different runs. This is mainly due to both alignment issues and spraying instabilities. Figure 5.24 presents the performance of the nanoelectrospray during a 2 hour period for different ion source operating pressures. Although the variations between needles can be significant, once the cone spray mode is achieved the system is very stable. Hysteresis effects were observed in the ion intensities due to changes in voltage or pressures in the ion source. As observed in the figure, the recovery period for most cases was around 10 to 20 mins. 


\section{Chapter 6}

\section{Summary and Conclusion}

If shown feasible, barium tagging in gaseous or liquid xenon would constitute an important technique in the detection of the neutrinoless double beta decay of ${ }^{136} \mathrm{Xe}$ as it would allow a measurement of the process in a background free environment and thus, with the operation of a large scale detector it would improve the sensitivity to probe the allowed region for the inverted neutrino mass hierarchy. Demonstrating the operation of a full working barium tagging system, in either gaseous or liquid xenon, within the time frame of the construction of the next phase EXO detector is thus one of the main priorities.

An ion extraction, transport, mass identification and counting system, to guide barium ions into a linear trap for laser induced fluorescence identification, was designed and developed. The implementation of a nanoelectrospray ionization source coupled to micron sized insulated nozzles for ion extraction from high pressure gas into vacuum of both singly and doubly charged states of barium was demonstrated. The dominant transfer of $\mathrm{Ba}^{+}$or $\mathrm{Ba}^{++}$was regulated by the ion kinetic energy control at the skimmer electrode. The whole technique was successful in minimizing the gas transfer into the system by limiting the gas flows down to $\sim 20 \mathrm{ml} / \mathrm{min}$. Ion transport into the quadrupole mass analyzer was achieved using a sextupole ion guide and an Einzel lens. Geometry and voltage parameters were optimized based on SIMION calculations to achieve barium ion intensities at the detector on the order of $\sim 1 \times 10^{5}$ counts per second for both barium charged states with mass resolutions of $\sim 0.1400$ mass units. 
Having an operational ion source of $\mathrm{Ba}^{+}$or $\mathrm{Ba}^{++}$will be of great utility in the development of both a charge conversion and a linear ion trap systems to perform fluorescence spectroscopy on the singly charged barium ion. The stability of the cone jet spraying mode producing $\sim 1 \times 10^{5}$ ions per second would be ideal and sufficient for the proof of principle of the barium tagging concept. The main limitations of the developed setup were the ion count reproducibility and the overall ion transfer efficiencies. The former, which presented variations within one order of magnitude, is attributed to the different initial conditions in the source. Needle-to-needle differences causing emitter-extractor geometry changes had an evident impact in the spraying mode operation, in the alignment, and thus in the detection and counting intensities. The latter, which is an important parameter to characterize the performance of the system, was not determined due to the unknown barium ion density in the sprayed solution. According to simulation calculations, the ion transport efficiencies within the ion optical path are expected to be $>70 \%$ in the best case scenarios; however, low efficiencies from the nanoelectrospray source are expected.

The ability to operate the ion source in nitrogen at pressures up to one bar and its positive effect in ion intensity, motivates the idea of pursuing studies for operation at higher pressures and in different noble gases (Ar, He, Xe) to emulate the conditions in the actual detector. Several tests with pressurized argon at 1 bar in the ion source were not successful due to continuous discharges at the nanoelectrospray emitter tip that caused ionization of the gas and no observation of barium ions in the detector. It is a subject of study to test the nanoelectrospray ion source in different gases at pressures higher than 1 bar.

A series of modifications to the ion source could be performed to address different studies necessary for the understanding of the feasibility of the barium tagging system. For example, a drift region can be designed to address questions regarding mobility of $\mathrm{Ba}^{+}$and $\mathrm{Ba}^{++}$ions in Xe at $\sim 10$ bar. Different barium ion sources, such as a ${ }^{252} C f$ fission source would exploit the advantages of having an ion counting detector at the end of the system and the efficiency of the setup could be determined. 
A proof of principle of barium tagging in gaseous Xenon coupled to the XEP prototype under construction at Carleton University is likely to be successful and will be determinant for the observation of neutrinoless double beta decay. The final system would extract the barium ion from the pressurized xenon vessel using the RF funnel ion extraction system under construction at Stanford University, the remaining amounts of xenon gas would be pumped out of the apparatus and the ion will be mass selected using the system developed in this work. Once mass identified, the ion would enter a hexapole collision cell where the presence of an electron donor buffer gas like TEA would convert the doubly charge ion into a singly charged one and the ion would be guided into a linear ion quadrupole trap where it would be identified using laser induced fluorescence. The ion trapping and laser system are currently under development at Carleton University. 


\section{References}

[1] J. Chadwick. Possible Existence of a Neutron. Nature 129, 312-312 (1932)

[2] C.L Cowan, F. Reines, F.B Harrison, H.W Kruse, and A.D McGuire. Detection of the Free Neutrino: a Confirmation. Science 124, 103-104 (1956)

[3] T.D Lee and C.N Yang. Question of Parity Conservation in Weak Interactions. Phys Rev Lett 104, 254-258 (1956)

[4] C.S. Wu, E. Ambler, R. W. Hayward, D.D. Hoppes and R.P Hudson. Experimental Test of Parity Conservation in Beta Decay. Phys Rev Lett 105, 1413-1415 (1957)

[5] M. Goldhaber, L. Grodzins, and A. W. Sunyar. Helicity of Neutrinos. Phys Rev Lett 109, 1015 - 1017 (1958)

[6] G. Danby, J.M Gaillard, K. A. Goulianos, L. M. Lederman, N. B. Mistry, M. Schwartz. Observation of High-Energy Neutrino Reactions and the Existence of Two Kinds of Neutrinos. Phys Rev Lett 9, 36-44 (1962)

[7] J.H. Christenson, J.W. Cronin, V.L Fitch, and R. Turlay. Evidence for the $2 \pi$ Decay of the $K_{2}^{0}$ Meson. Phys Rev Lett 13, 138-140 (1964)

[8] M. L. Perl et al. Evidence for Anomalous Lepton Production in $e^{+}-e^{-}$Annihilation. Phys Rev Lett 35, 1489-1492 (1975)

[9] B. Aubert et al [BaBar collaboration]. Direct CP Violating Asymmetry in $B^{0} \rightarrow$ $K^{+} \pi^{-}$Decays. Phys Rev Lett 93, 131801 (2004)

[10] Y. Chao et al [Belle collaboration]. Evidence for Direct CP Violation in $B^{0} \rightarrow$ $K^{+} \pi^{-}$Decays . Phys Rev Lett 93, 191802 (2004) 
[11] R.J Davis, D. S Harmer, and K. C. Hoffman. Search for Neutrinos from the Sun. Phys Rev Lett 20, 1205-1209 (1968)

[12] J.N. Bahcall. Solar Neutrinos. I. Theoretical. Phys Rev Lett 12, 300-303 (1964)

[13] J. N. Bahcall, N. A. Bahcall, and G. Shaviv. Present Status of the Theoretical Predictions for the ${ }^{37} \mathrm{Cl}$ Solar-Neutrino Experiment. Phys Rev Lett 20, 1209$1212(1968)$

[14] Davis Jr., R., L. C. Rogers, V. Radeka. Report on the Brookhaven solar neutrino experiment. Bull. Am. Phys. Soc. 16, 631-633 (1971)

[15] B. Pontercovo. Neutrino Experiments and the Problem of Conservation of Leptonic Charge. Sov. Phys JETP 26, 984-988 (1968)

[16] B. Aharmin et al [SNO collaboration]. Measurement of the Rate of $\nu_{e}+d \rightarrow$ $p+p+e^{-}$Interactions Produced by ${ }^{8} B$ Solar Neutrinos at the Sudbury Neutrino Observatory. Phys Rev Lett 87, 071301, 6 pages (2001)

[17] Gell-Mann, Murray et al. Behavior of neutral particles under charge conjugation. Phys Rev Lett 97, 1387-1389 (1955)

[18] Z. Maki, M. Nakagawa, and S. Sakata. Remarks on the Unified Model of Elementary Particles. Prog. Theor. Phys 28, 870-880 (1962)

[19] C. Guinti, Chung W. Kim. Fundamentals of neutrino physics and astrophysics. Oxford University Press Inc., New York (2007)

[20] K. Kodama et al [DONUT Collaboration]. Observation of Tau Neutrino Interactions. Phys Rev Lett B 504, 218-224 (2001)

[21] J. Beringer et al (Particle Data Group Collaboration). Review of Particle Physics. Phys Rev Lett D 86, 010001 (2013)

[22] K.H Ackerman et al (GERDA collaboration). The GERDA experiment for the search of $0 \nu \beta \beta$ decay in ${ }^{76}$ Ge. Eur. Phys. J. C 73 2330, 29 pages (2013) 
[23] KamLAND Zen Collaboration. Measurement of the double- $\beta$ decay half-life of ${ }^{136} \mathrm{Xe}$ with the KamLAND-Zen experiment. Phys Rev C 85, 045504, 6 pages (2012)

[24] F. Alessandria et al. (CUORE collaboration). Sensitivity and Discovery Potential of CUORE to Neutrinoless Double-Beta Decay. arXiv:1109.0494v3, 14 pages [nucl-ex]

[25] S.R. Elliott et al (MAJORANA collaboration). The MAJORANA DEMONSTRATOR: A Search for Neutrinoless Double-beta Decay of Germanium-76. [arXiv:1307.7777v1], 4 pages (2013)

[26] Boehm F, Vogel P. Physics of Massive Neutrinos. Cambridge University Press, Cambridge. (1992)

[27] V.N. Aseev et al. An upper limit on electron antineutrino mass from Troitsk experiment. Phys Rev D 84, 112003, 9 pages (2011)

[28] K. Assamagan et al. Upper limit of the Muon-Neutrino Mass and ChargedPion Mass from Momentum Analysis of a Surface Muon Beam. Phys Rev D 53, 6065-6077 (1996)

[29] R. Barate et al (ALEPH Collaboration). An Upper limit on the tau-neutrino mass from three-prong and five-prong tau decays. Eur Phys. J. C 2, 395-406 (1998)

[30] P. Bamert, C. P. Burgess, and R. N. Mohapatra. Multi-Majoron Modes for Neutrinoless Double-Beta Decay. Nucl. Phys. B 449, 25-48 (1995).

[31] H.V. Klapdor-Kleingrothaus et al. Evidence for Neutrinoless Double Beta Decay. Mod Phys Lett A 16, 2409-2420 (2001).

[32] R. N. Mohapatra, A. Perez-Lorenzana, and C. A. de S. Pires. Neutrino mass, bulk majoron and neutrinoless double beta decay. Phys Rev Lett B 491, 143-147 $(2000)$.

[33] S. M. Bilenky, C. Giunti. Neutrinoless double-beta decay. A brief review. Mod Phys Lett A 27, 1230015, 22 pages (2012) 
[34] N. Ackerman et al (EXO collaboration). Observation of Two-Neutrino DoubleBeta Decay in ${ }^{136} \mathrm{Xe}$ with the EXO-200 Detector. Phys Rev Lett 107, 212501, 5 pages $(2011)$

[35] J.B. Albert et al (EXO collaboration). An improved measurement of the $2 \nu \beta \beta$ half-life of Xe-136 with EXO-200. [arXiv:1306.6106v3], 34 pages (2013)

[36] A. Gando et al (KamLAND-Zen Collaboration). Limit on Neutrinoless $\beta \beta$ Decay of 136Xe from the First Phase of KamLAND-Zen and Comparison with the Positive Claim in 76Ge. Phys Rev Lett 110, 062502, 5 pages (2013)

[37] E. I. Esch et al. The Cosmic Ray Muon Flux at WIPP. Nucl. Inst. and Meth A 538, 516-525 (2005)

[38] M. Auger et al (EXO collaboration). The EXO-200 detector, part I: Detector design and construction. JINST 7 P05010, 43 pages (2012)

[39] Kubota, S., M. Hishida, and J.Ruan. Evidence for a triplet state of the selftrapped exciton states in liquid argon, krypton and xenon. J. Physics 11, 26452651 (1978)

[40] K. Graham. Searching for Neutrinoless Double Beta Decay with EXO. WNPPC, Mont Tremblant (2012)

[41] A. Bolotnikov et al. The spectroscopic properties of high pressure xenon. Nucl. Ins. Meth A 396, 360-370 (1997)

[42] R. Luscher et al. Search for beta beta decay in Xe-136: New results from the Gotthard experiment. Phys Rev Lett B 434, 407-414 (1998)

[43] Yahlali N et al. NEXT: Neutrino Experiment with high pressure Xenon gas TPC. Nucl. Instrum. Meth. Phys. A 617, 520-522 (2010)

[44] V. Alvarez et al (NEXT collaboration). Near-Intrinsic Energy Resolution for 30 to $662 \mathrm{keV}$ Gamma Rays in a High Pressure Xenon Electroluminescent TPC. Nucl.Instrum.Meth. A708, 101-114 (2013)

[45] M.K. Moe. Detection of neutrinoless double-beta decay. Phys Rev C 44, R931-R934 (1991) 
[46] M. Danilov et al. Detection of very small neutrino masses in double-beta decay using laser tagging. Phys. Lett. B 480, 12-18 (2000)

[47] W. Neuhauser, M. Hohenstatt, P. Toschek, H. Dehmelt. Optical-Sideband Cooling of Visible Atom Cloud Confined in Parabolic Well. Phys Rev Lett 41 233-236 (1978)

[48] J. J. Curry. Compilation of Wavelengths, Energy Levels, and Transition Probabilities for Ba I and Ba II. Journal of Physical and Chemical Reference Data 33 (3), 725-746 (2004)

[49] E. Rollin. Barium Ion Extraction and Identification from Laser Induced Fluorescence in Gas for the Enriched Xenon Observatory. PhD Thesis, Department of Physics, Carleton University 2011.

[50] V.L. Varentsov and M. Wada. Computer experiments on ion beam cooling and guiding in fair-wind gas cell and extraction RF-funnel system. Nucl. Instr. and Meth. A 532, 210-215 (2004)

[51] A. Gallagher. Oscillator Strengths of Ca II, Sr II, and Ba II. Phys Rev Lett 157, 24-30 (1967)

[52] M. Green, J. Wodin, R. deVoe, P. Fierlinger et al. Observation of single collisionally cooled trapped ions in a buffer gas. Phys Rev A 76, 023404, 5 pages (2007)

[53] B. Flatt et al. A linear RFQ ion trap for the Enriched Xenon Observatory. Nucl. Instr. and Meth. A 578, 399-392 (2007)

[54] D. Sinclair et al. Prospects for Barium Tagging in Gaseous Xenon. J. Phys.: Conference Series 309, 012005, 8 pages (2011)

[55] T. Brunner et al. A setup for Ba-ion extraction from high pressure Xe gas for double-beta decay studies with EXO. [arXiv:1302.6940v1], 5 pages (2013)

[56] Gerlich, D. Inhomogeneous RF fields: a versatile tool for the study of processes with slow ions. John Wiley \& Sons, New York (1992) 
[57] Raymond E. March, John F. Todd. Quadrupole Ion Trap Mass Spectrometry. John Wiley \& Sons, Hoboken (2005)

[58] D.J. Douglas, A. J. Frank, D. Mao. Linear Ion Traps in Mass Spectrometry. Mass Spectrometry Reviews 24, 1-29 (2005)

[59] Raymond E. March, John F. J. Todd, Richard M. Hughes. Quadrupole storage mass spectrometry. John Wiley \& Sons, New York (1989)

[60] N.W. McLachan. Theory and Applications of Mathieu Functions. Clarendon, Oxford (1947)

[61] J. J. Everdij, A. Huijser and N. F. Verster. Improved Space Focusing of Polar Diatomic Molecules in a System of Quadrupole and Hexapole Fields. Rev. Sci. Instrum. 44, 721-725 (1973)

[62] Rama Rao VVK, Bhutani A. Electric hexapoles and octopoles with optimized circular section rods. Int J Mass Spectrometry 202, 31-36 (2000)

[63] S. Banerjee, S. Mazumdar. Electrospray Ionization Mass Spectrometry: A Technique to Access the Information beyond the Molecular Weight of the Analyte. International Journal of Analytical Chemistry 2012, 282574, 40 pages (2012)

[64] P. Gates. Electrospray Ionization. University of Bristol (2004)

[65] J. Zeleny. The electrical discharge from liquid points, and a hydrostatic method of measuring the electric intensity at their surfaces. Phys Rev Lett 3, 69-91 (1914)

[66] G. Taylor. Disintegration of water drops in an electric field. Proceedings of the Royal Society of London. Series A, Mathematical and Physical Sciences 280, 383-397 (1964).

[67] S. R. Elliott and P. Vogel, Double Beta Decay, Ann. Rev. Nucl. Part. Sci. 52, 115-151 (2002)

[68] Tang, L.; Kebarle, P. Dependence of ion intensity in electrospray mass spectrometry on the concentration of analytes in the electrosprayed solution. Anal. Chem. 65, 3654- 3668 (1993) 
[69] U. Bahr, A. Pfenninger, M. Karas, B. Stahl. High sensitivity analysis of neutral underivatized oligosaccharides by nanoelectrospray mass spectrometry. Anal. Chem. 69, 4530- 4535 (1997)

[70] M. S. Wilm, M. Mann. Analytical Properties of the Nanoelectrospray Ion Source. Anal. Chem 68, 1-8 (1996)

[71] Perry, Robert H. and Green, Don W. Perry's Chemical Engineers' Handbook. McGraw Hill, New York. (1984)

[72] Fernandez de la Mora, et al. The Current Emitted by Highly Conducting Taylor Cones. J. Fluid Mech 260, 155-184 (1994)

[73] R. J. Pfeifer, C. D. Hendricks. Parametric Studies of Electrohydrodynamic Spraying. AIAA J. 6, 496-502 (1968)

[74] M.S. Wilm, M. Mann. Electrospray and Taylor-Cone theory, Dole's beam of macromolecules at last? Int. J. Mass Spectrometry. Ion Processes 136, 167-180 (1994)

[75] J. Fernández De La Mora. The effect of charge emission from electrified liquid cones. Journal of Fluid Mechanics 243, 561-574 (1992)

[76] Fernández de la Mora. Fluid dynamics of electrosprays. J. Annual Review of Fluid Mechanics 39, 217-243 (2007).

[77] M. Cloupeau and B. Prunet-Foch. Electrostatic spraying of liquids in cone-jet mode. Journal of Electrostatics 22, 135-159 (1989)

[78] Smith, D. P. H., The electrohydrodynamic atomization of liquids. IEEE Transactions on Industry Applications 22, 527-535 (1986).

[79] Lenggoro, I. W., Okuyama, K., Fernández de la Mora, J., and Tohge, N. Preparation of $\mathrm{ZnS}$ nanoparticles by electrospray pyrolysis. Journal of Aerosol Science 31, 121-136 (2000)

[80] R.P.A. Hartman et al. Jet break-up in electrohydrodynamic atomization in the cone-jet mode. Journal of Aerosol Science 31 (1), 65-95 (2000) 
[81] Extrel Detector Manual 815181b. Extrel CMS, LLC Pittsburgh, PA (2012) 